\title{
Attenuation of oxidative stress-induced lesions in skeletal muscle in a mouse model of obesity-independent hyperlipidaemia and atherosclerosis through the inhibition of Nox2 activity
}

Article

Accepted Version

Creative Commons: Attribution-Noncommercial-No Derivative Works 4.0

Sfyri, P. P., Yuldasheva, N. Y., Tzimou, A., Giallourou, N., Crispi, V., Aburima, A., Beltran-Alvarez, P., Patel, K., Mougios, V., Swann, J. R., Kearney, M. T. and Matsakas, A. (2018) Attenuation of oxidative stress-induced lesions in skeletal muscle in a mouse model of obesity-independent hyperlipidaemia and atherosclerosis through the inhibition of Nox2 activity. Free Radical Biology \& Medicine, 129. pp. 504519. ISSN 0891-5849 doi: https://doi.org/10.1016/j.freeradbiomed.2018.10.422 Available at https://centaur.reading.ac.uk/79868/

It is advisable to refer to the publisher's version if you intend to cite from the work. See Guidance on citing.

To link to this article DOI: http://dx.doi.org/10.1016/j.freeradbiomed.2018.10.422 
All outputs in CentAUR are protected by Intellectual Property Rights law, including copyright law. Copyright and IPR is retained by the creators or other copyright holders. Terms and conditions for use of this material are defined in the End User Agreement.

\section{www.reading.ac.uk/centaur}

\section{CentAUR}

Central Archive at the University of Reading

Reading's research outputs online 


\section{Title page}

\section{Research article}

Attenuation of oxidative stress-induced lesions in skeletal muscle in a mouse model of obesity-independent hyperlipidaemia and atherosclerosis through the inhibition of Nox2 activity

Pagona Panagiota Sfyri ${ }^{1}$, Nadira Y. Yuldasheva ${ }^{2}$, Anastasia Tzimou ${ }^{3}$, Natasa Giallourou ${ }^{4}$, Vassili Crispi ${ }^{1}$, Ahmed Aburima ${ }^{1}$, Pedro Beltran-Alvarez ${ }^{5}$, Ketan Patel ${ }^{6}$, Vassilis Mougios ${ }^{3}$, Jonathan R. Swann ${ }^{4}$, Mark T. Kearney ${ }^{2}$, Antonios Matsakas ${ }^{1}$

${ }^{1}$ Molecular Physiology Laboratory, Centre for Atherothrombotic \& Metabolic Disease, Hull York Medical School;

${ }^{2}$ Leeds Institute of Genetics Health and Therapeutics, University of Leeds;

${ }^{3}$ Laboratory of Evaluation of Human Biological Performance, School of Physical Education and Sports Science at Thessaloniki, Aristotle University of Thessaloniki;

${ }^{4}$ Department of Surgery and Cancer, Division of Computational and Systems Medicine, Imperial College London;

${ }^{5}$ School of Life Sciences, University of Hull;

${ }^{6}$ School of Biological Sciences, University of Reading

\section{Correspondence:}

Dr. Antonios Matsakas

Molecular Physiology Laboratory

Centre for Atherothrombotic \& Metabolic Disease

Hull York Medical School

University of Hull

Cottingham Road

Hull, HU6 7RX

United Kingdom

Tel: $+44(0) 1482465008$

Email: Antonios.Matsakas@hyms.ac.uk 


\section{Abstract}

Obesity leading to hyperlipidaemia and atherosclerosis is recognised to induce morphological and metabolic changes in many tissues. However, both-hyperlipidaemia and atherosclerosis can occur in the absence of obesity. The impact of the latter scenario on skeletal muscle and liver is not understood sufficiently. In this regard, we used the Apolipoprotein E-deficient $\left(\mathrm{ApoE}^{-/-}\right)$mouse model, an established model of hyperlipidaemia and atherosclerosis, that does not become obese when subjected to a high-fat diet, to determine the impact of Western-type diet (WD) and ApoE deficiency on skeletal muscle morphological, metabolic and biochemical properties. To establish the potential of therapeutic targets, we further examined the impact of Nox2 pharmacological inhibition on skeletal muscle redox biology. We found ectopic lipid accumulation in skeletal muscle and the liver, and altered skeletal muscle morphology and intramuscular triacylglycerol fatty acid composition. WD and ApoE deficiency had a detrimental impact in muscle metabolome, followed by perturbed gene expression for fatty acid uptake and oxidation. Importantly, there was enhanced oxidative stress in the skeletal muscle and development of liver steatosis, inflammation and oxidative protein modifications. Pharmacological inhibition of Nox2 decreased reactive oxygen species production and protein oxidative modifications in the muscle of $\mathrm{ApoE}^{-/-}$mice subjected to a Western-type diet. This study provides key evidence to better understand the pathophysiology of skeletal muscle in the context of hyperlipidaemia and atherosclerosis and identifies Nox2 as a potential target for attenuating oxidative stress in skeletal muscle in a mouse model of obesity-independent hyperlipidaemia and atherosclerosis.

Key words: Atherosclerosis, Western-type diet, NADPH oxidase, skeletal muscle, oxidative stress 
Abbreviations: 2-OH-E+: 2-hydroxyethidium; 3NT: 3-nitrotyrosine; 4HNE: 4-hydroxy-2nonenal; 8OH-dG: 8-hydroxy-2'-deoxyguanosine; ApoE ${ }^{-/-}$: Apolipoprotein E knockout; CD36: Cluster of differentiation 36; CSA: Cross-sectional area; DHE: Dihydroethidium; EDL: Extensor digitorum longus; F4.80: EGF-like module-containing mucin-like hormone receptorlike 1; $\mathrm{H}_{2} \mathrm{O}_{2}$ : Hydrogen peroxide; LDL: Low density lipoprotein; MHC: Myosin heavy chain; ND: Normal diet; Nox: Nicotinamide adenine dinucleotide phosphate oxidase; Nox2ds-tat: Nox2 docking sequence-tat; $\mathrm{O}_{2} \cdots$ : Superoxide; PAD: Peripheral arterial disease; ROS: Reactive oxygen species; TA: Tibialis anterior; TG: Triacylglycerols; Tnfa: Tumour necrosis factor $\alpha$; WD: Western-type diet; WT: Wild type

\section{Introduction}

Hyperlipidaemia is an established risk factor for atherosclerosis and cardiovascular disease [1]. Most studies have shown that obesity per se induces elevated levels of circulating free fatty acids - deriving mainly from adipose tissue - that lead to ectopic fat accumulation in the liver and skeletal muscle [2]. Evidence also suggests that obesity-dependent hyperlipidaemia is a risk factor for non-alcoholic fatty liver disease and adipose tissue inflammation, conditions that in turn are associated with atherosclerosis [3, 4]. However, the obesity-independent impact of hyperlipidaemia and atherosclerosis on metabolically active tissues has only recently begun to be unravelled $[5,6]$.

Atherosclerosis and hyperlipidaemia may also exert effects on skeletal muscle $[7,8]$. For example, the effect of atherosclerosis and hyperlipidaemia on skeletal muscle morphology and function has been investigated in the context of peripheral arterial disease (PAD). PAD is characterised by vascular deficits leading to restriction and blockage of the arteries in the lower extremities and myopathy [9]. PAD myopathy is characterised by increased oxidative damage, altered myofibre morphology, desmin accumulation, loss of force generating 
capacity and mitochondrial deficits [10-12]. However, many PAD patients exhibit comorbidities, such as hypertension and type II diabetes that may have a modifying role in skeletal muscle pathophysiology.

In this regard, sparse evidence from the $\mathrm{ApoE}^{--}$mouse suggests that obesity-independent hyperlipidaemia and atherosclerosis may have an impact on skeletal muscle physiology [1316]. The $\mathrm{ApoE}^{-/-}$mouse is an established model of atherosclerosis that develops hyperlipidaemia and atherosclerotic lesions from the age of 3-4 months accompanied by increased oxidative stress [17]. Administration of a high-fat diet in $\mathrm{ApoE}^{-/-}$mice further increases plasma cholesterol and promotes earlier lesion development [17]. In addition, $\mathrm{ApoE}^{-/-}$mice on either a high-fat diet or a Western-type diet (i.e. high-fat with cholesterol) show a modest increase in body weight compared to wild-type mice $[16,18]$. Limited data on skeletal muscle of $\mathrm{ApoE}^{-/-}$mice reported reduced capillary density and increased lipid peroxidation products, suggesting that hyperlipidaemia and possibly atherosclerosis may impact on skeletal muscle biology and redox homeostasis [13, 14].

NADPH oxidases (Noxs) are reactive oxygen species (ROS) generating enzymes and their role in physiological cellular responses as redox mediators is currently being investigated [19]. In particular, Nox2 contributes to physiological responses, such as wound healing, and is also involved in pathological conditions, such as atherosclerosis [20]. For example, Nox2 deficiency in $\mathrm{ApoE}^{-/-}$mice led to smaller atherosclerotic lesion area in the aorta [21]. However, the impact of Nox2 on skeletal muscle of $\mathrm{ApoE}^{-/-}$mice has not been established. Nox2 is considered an important source of ROS in response to skeletal muscle contraction and mechanotransduction as well as in insulin signalling [22]. In wild-type mice under a highfat diet, Nox2 protein expression and activity are increased in skeletal muscle, suggesting that dietary challenges may represent a key independent factor that may lead to oxidative stress [23-25]. Since Nox2-derived ROS production is increased under dietary challenges, inhibition of Nox2 may be a useful means to attenuate skeletal muscle oxidative stress. Nox2ds-tat is a chimeric 18-amino acid peptide that inhibits Nox2 enzymatic activity and is 
known to suppress superoxide production in endothelial cells [26]. Specifically, the Nox2dstat peptide inhibitor has been shown to decrease ROS production and oxidative stress and improve vasorelaxation and nitric oxide production in arteries of rodents [27-29]. Furthermore, treatment of diet-induced obese mice with the Nox2ds-tat peptide inhibitor after femoral artery ligation accelerated collateral growth and hindlimb reperfusion [30]. Accordingly, inhibition of Nox2 with the Nox2ds-tat peptide in $\mathrm{ApoE}^{--}$mice fed a Westerntype diet, resulted in decreased atherosclerotic lesions throughout the aorta and the carotid arteries [31]. However, the impact of Nox2 in vivo inhibition on skeletal muscle oxidative stress has not been studied so far.

In this study we hypothesised that hyperlipidaemia and atherosclerosis would have a negative impact on skeletal muscle and liver homeostasis due to the production of ROS, a situation worsened by a Western-type diet. Our main findings are that $\mathrm{ApoE}^{-/-}$mice exhibit ectopic fat accumulation in both skeletal muscle and the liver, with hepatic steatosis being aggravated by Western-type diet. Intramyocellular lipid accumulation leads to enlarged myofibres and to a possible functional adaptation characterised by a transition from fasttwitch to slower fibre type and enhanced capillary density. Nonetheless, this remodelling is not adequate to prevent oxidative stress as shown by enhanced ROS production, perturbed antioxidant gene expression and oxidative modifications. Interestingly, administration of a Western-type diet did not reveal any synergistic effect with genotype for the majority of the parameters studied (e.g. myofibre size, capillary density and oxidative stress). Most importantly, Nox2 seems to contribute to skeletal muscle oxidative stress under hyperlipidaemic and atherosclerotic conditions, since pharmacological inhibition of Nox2 resulted in attenuation of oxidative stress.

\section{Materials and methods}

Detailed experimental methods are provided in the online supplement. 


\section{Animal Maintenance}

Male C56BI/6J (wild-type, WT) and $\mathrm{ApoE}^{--}$mice on the same genetic background were housed under standard environmental conditions $\left(20-22^{\circ} \mathrm{C}, 12-12 \mathrm{hr}\right.$ light-dark cycle) and were provided standard chow (normal diet, ND) and water ad libitum. At the age of 8 weeks some mice from both genotypes were switched to a Western-type diet (WD, composed of $21 \%(w / w)$ fat and supplemented with $0.15 \%(w / w)$ cholesterol; Special Diet Services \#829100; Braintree, UK) for 12 weeks. A subgroup of $\mathrm{ApoE}^{-/-}$mice was fed the Western-type diet for 8 weeks before being implanted with an osmotic minipump (model 1004, ALZET, Charles River, UK) at the back of the animal that was prefilled with either Nox2ds-tat (RKKRRQRRRCSTRIRRQL - NH2) or Nox2ds-tat scrambled (RKKRRQRRRCLRITRQSR $\mathrm{NH} 2$ ) to provide a dose of $10 \mathrm{mg} / \mathrm{kg} /$ day i.p. as described previously [32]. Animals were kept on the Western-type diet for another 4 weeks, a total of 12 weeks. Each experimental group consisted of 5-6 mice.

The experiments were performed under a project license from the United Kingdom Home Office in agreement with the revised Animals (Scientific Procedures) Act 1986. The University of Leeds Animal Care and Ethical Review Committee approved all procedures. Animals were humanely sacrificed via Schedule 1 killing under terminal anaesthesia between 9:00-13:00.

\section{Immunohistochemistry}

Immunohistochemistry was performed on $10 \mu \mathrm{m}$ cryosections as described previously [33]. Antibodies against myosin heavy chain isoforms, cluster of differentiation 31 (CD31), 3nitrotyrosine (3NT), laminin, 8-hydroxy-2'-deoxyguanosine (8OH-dG) and E06 were used. Details of primary and secondary antibodies are given in Supplementary Table 1. F4.80 was detected using the Vector Laboratories ImmPRESS Excel Staining Kit. 


\section{Histology}

Succinate dehydrogenase $(\mathrm{SDH})$ staining of extensor digitorum longus (EDL), tibialis anterior (TA) and soleus was performed as described previously [34]. Similarly, for Oil Red O (Sigma-Aldrich, UK) and Bodipy 493/503 (ThermoFisher Scientific, USA) staining transverse muscle sections were fixed in $4 \%$ paraformaldehyde, incubated with either $75 \%$ Oil Red 0 working Solution or $20 \mu \mathrm{g} / \mathrm{mL}$ Bodipy $493 / 503$ solution for 30 min and subsequently mounted with hydromount mounting medium (Fisher Scientific, UK). Masson's trichrome staining was used to detect tissue fibrosis following the manufacturer's instructions (EMD Millipore, USA).

\section{Morphometrics}

Images were captured using an Axiolmager fluorescence microscope and Axiocam digital camera. Coloured mages were captured with a Zeiss Axiom 105 microscope camera, coupled with ZEN imaging software (Zeiss, Germany). Quantification of myofibre type and cross-sectional area (CSA) measurements were performed manually using Zen software. All fibres negative for MHC IIA and IIB on double-stained sections were considered as type IIX, upon subtraction of the type I stained fibres from serial sections.

\section{Quantitative PCR}

Quantitative PCR was performed as described previously [34]. Total RNA was prepared from skeletal muscles using the EZNA Total RNA Kit I (Omega Biotek, USA). Total RNA $(5 \mu \mathrm{g})$ was reverse-transcribed to cDNA with RevertAid H MinusFirst Strand cDNA synthesis kit (ThermoFisher Scientific, USA) and analysed by quantitative real-time RT-PCR on a StepOne Plus cycler (Applied Biosystems, UK), using the Applied Biosystems SYBRGreen PCR Master Mix. Details of primers are given in Supplementary Table 3. Relative expression was calculated using the $\Delta \Delta \mathrm{Ct}$ method with normalisation to the housekeeping genes encoding cyclophilin-B (Сyp) and hypoxanthine-guanine phosphoribosyltransferase (Hprt). 


\section{SDS PAGE and Immunoblotting}

Frozen muscles and liver were homogenised in RIPA lysis buffer supplemented with protease inhibitors. Protein content was quantified using the Pierce ${ }^{\mathrm{TM}}$ BCA Protein Assay Kit (ThermoFisher Scientific, USA). $30 \mu \mathrm{g}$ of muscle or liver protein extracts were used for immunoblotting as described previously [32, 34]. Details of antibodies are given in Supplementary Table 2. For detection of protein carbonyls the Oxidized Protein Western Blot Detection Kit (Abcam, UK) was used according to the manufacturer's instructions. Densitometric analysis was performed on ImageJ Software.

\section{Intramuscular lipid contents by gas chromatography}

Intramuscular triacylglycerols and phospholipids were analysed by a combination of thinlayer chromatography and gas chromatography on an Agilent 7890A gas chromatograph (Santa Clara, CA), equipped with a 30 m-long AT-WAX capillary column (Alltech, Deerfield, IL) and flame ionization detector, as described previously [35].

\section{${ }^{1} \mathrm{H}$ nuclear magnetic resonance spectroscopy-based (NMR) metabonomic analysis} Aqueous metabolites were extracted from gastrocnemius muscle as described in previous protocols [36, 37]. Principal components analysis (PCA) was performed with Pareto scaling in MATLAB using in-house scripts.

\section{TBARS and protein carbonyls}

Thiobarbituric Acid Reactive Substances (TBARS) were evaluated with the OXItek TBARS Assay Kit (Enzo Life Sciences, USA) according to the manufacturer's instructions. Protein 
carbonyl content was assayed in whole muscle and liver lysates with the Protein Carbonyl Content Assay Kit (Sigma-Aldrich, UK) according to the manufacturer's instructions.

\section{Liquid Chromatography Mass Spectrometry (LC-MS) detection of 2-hydroxyethidium}

Muscle tissue sections were incubated with $5 \mu \mathrm{mol} / \mathrm{L}$ of dihydroethidium (DHE) in PBS, for 30 min at $37^{\circ} \mathrm{C}$ and were subsequently washed with PBS-diethylenetriaminepentaacetic acid (DTPA). LC-MS analysis for skeletal muscle was performed as described previously [32].

\section{Statistical analysis}

The results in this study are reported as mean $\pm S D$. This study took into consideration the ARRIVE guidelines for reporting animal research [38]. Sample size was determined by using the $G^{*}$ Power software to achieve $80 \%$ power in any given parameter at the $p<0.05$ significance level [39]. Significant differences among groups for dependent variables were detected by using two-way ANOVA (genotype $x$ diet) followed by the Bonferroni post hoc tests when a main effect or interaction was significant. Differences between two groups (i.e. $\mathrm{ApoE}^{-/-}$Nox2ds-tat versus $\mathrm{ApoE}^{-/-}$scrambled peptide) were detected by the Student's $\mathrm{t}$ test. Non-parametric tests were used if the data did not pass the Shapiro-Wilk normality test as indicated in the figure legends. Chi square $\left(x^{2}\right)$ test was used to detect differences in lipid droplet size and CSA. Significant differences were considered for $p<0.05$. Statistical analysis was performed in GraphPad Prism 6 (two-way ANOVA and Mann-Whitney U test) or IBM SPSS software version 24 (chi-square test).

\section{Results}

The impact of Western-type diet and ApoE deficiency on skeletal muscle morphological properties 
Muscle fibre typing: The effect of systemic atherosclerosis and hyperlipidaemia on skeletal muscle features of the $\mathrm{ApoE}^{-/-}$mouse remains largely unexplored. Therefore, we assessed the skeletal muscle fibre composition and size in wild-type and $\mathrm{ApoE}^{-/-}$mice subjected to either a standard chow diet (normal diet, ND) or a Western-type diet (WD) for 12 weeks. Fibre type composition of the superficial TA muscle was unaltered amongst groups (Fig. 1AB). However, there was a $20 \%$ decrease of IIB fibres in the deep region of the TA in response to ApoE deficiency, without any changes in WT WD mice (Fig. 1A-B). A cumulative effect was found when $\mathrm{ApoE}^{-/-}$mice were administered a WD for 12 weeks, resulting in a $30 \%$ decrease of IIB fibres compared to WT ND (Fig. 1A-B). Decrease in the proportion of IIB fibres was accompanied by increase of IIX fibres that reached levels of significance in the $\mathrm{ApoE}^{--} \mathrm{ND}$ and $\mathrm{ApoE}^{-/-} \mathrm{WD}$ compared to WT ND. To expand the significance of this finding, the EDL and soleus fibre type composition was also examined. We found a $10 \%$ decrease of IIB fibres in EDL of $\mathrm{ApoE}^{--}$ND compared to WT ND without a synergistic effect when $\mathrm{ApoE}^{-/-}$mice were subjected to Western-type diet (Supplementary Fig. 1). Similarly, there was a significant drop in IIB fibres in the soleus of all groups compared to WT ND, while ApoE ${ }^{-/-}$ND mice showed an increase in the proportion of slowtwitch type I fibres (Supplementary Fig. 1).

Metabolic profiling of skeletal muscle: Given the partial fibre type transition in response to ApoE deficiency and Western-type diet, the proportion of SDH positive fibres and mitochondrial DNA content was assessed as a measure of muscle mitochondrial activity and mitochondrial biogenesis respectively. SDH positive fibres were significantly higher in all groups compared to WT ND in the EDL, but not in the other muscles studied (superficial and deep TA, and soleus). There was not any compromise in skeletal muscle mitochondrial biogenesis in response to either ApoE deficiency or Western-type diet (Supplementary Fig. 2).

Muscle fibre area: Cross-sectional area analysis of specific fibre types in superficial and deep TA as well as the EDL and the soleus muscles showed differences in fibre size. Type 
IIX fibres were significantly larger in superficial TA for all experimental groups compared to WT ND (Fig. 1C-D). Similarly, there were significantly larger IIX fibres in the deep TA of $\mathrm{ApoE}^{-/-}$independent of diet, while IIB fibres were significantly smaller in response to Western-type diet for both genotypes as compared to WT ND (Fig. 1C-D). Larger IIA fibres were also found in $\mathrm{ApoE}^{-/-}$WD compared to WT and $A p o E^{-/-}$ND. Consistent with the increased fibre size in the TA, we found increased cross-sectional area of IIA and IIX fibres in EDL as well as IIA in soleus for all three groups compared to WT ND (Supplementary Fig. 1). In addition, IIB fibres were significantly larger in the $E D L$ muscle in $A p o E^{-/-}$mice independent of diet as compared to WT ND. Finally, diet had an impact on the size of type I fibres in soleus independent of genotype.

Body mass: There was no difference in body weight at baseline between WT ND and ApoEI- ND. However, 12 weeks of Western-type diet induced an almost three times-higher increase of body weight in wild-type mice compared to $\mathrm{ApoE}^{-/-}$(i.e. 35 vs. $15 \%$ body weight increase in WT WD and ApoE ${ }^{-/-}$WD respectively; Supplementary Fig. 1).

Muscle capillary density and fibrosis: It is known that oxidative myofibres are surrounded by more capillaries than glycolytic fibres to support their metabolic activity [40]. Given the IIB fibre transition to IIX, a slower phenotype, capillary density was next determined in EDL and TA muscles. CD31 staining revealed higher capillary-to-fibre ratio of $20 \%$ and $15 \%$ in EDL and superficial TA respectively in response to diet and genotype compared to WT ND mice (Fig. 2A-B). This increase was accompanied by increased mRNA levels of the angiogenic genes Vegfa165 and Fgf1 in gastrocnemius muscle (Fig. 2C). High-fat diet has been associated with extracellular matrix remodelling and collagen deposition in skeletal muscle $[41,42]$. Therefore, the potential of skeletal muscle fibrosis was examined in the TA by Masson's trichrome staining. We did not find any evidence of muscle fibrosis in any of the experimental groups studied (Supplementary Fig. 3A). Our findings suggest that neither Western-type diet nor ApoE deficiency induce skeletal muscle fibrosis. 
The effect of Western-type diet and ApoE deficiency on intramuscular fat contents and skeletal muscle metabolic signature

Intramuscular fat contents: $\mathrm{ApoE}^{-/-}$mice are hyperlipidaemic showing increased blood triacylglycerol and cholesterol levels that are further increased in response to a Western-type diet $[17,18]$. Given the increased capillary density in skeletal muscle of $\mathrm{ApoE}^{-/-}$mice, we hypothesised that excessive fat may be available for uptake and ectopic deposition. Transcript levels of genes that regulate fatty acid uptake in skeletal muscle, i.e. Cluster of differentiation $36(\mathrm{Cd} 36)$ and Fatty acid transport protein 1 (Fatp1), two major fatty acid transporters and Cell death activator (Cidea), a regulator of triacylglycerol deposition, were found consistently higher in all experimental groups compared to WT ND mice in gastrocnemius muscle (Fig. 3A). There was a profound CD36 staining both in the cytoplasm and on the plasma membrane of fibres other than type IIB, indicating a robust upregulation both at transcript and protein levels of this fatty acid transporter (Fig. 3B). Intramyocellular fat contents were increased in WT WD, ApoE ${ }^{-/-} \mathrm{ND}$ and $A p o E^{-/-}$WD compared to WT ND as evidenced by Bodipy 493/503 (Fig. 3C) and Oil red O staining (Supplementary Fig. 3B). Quantification of muscle triacylglycerol contents with gas chromatography revealed 400$500 \%$ higher total triacylglycerol levels in WT WD, $\mathrm{ApoE}^{-/-} \mathrm{ND}$ and $\mathrm{ApoE}^{-/-}$WD compared to WT ND (Fig. 3D). In particular, there were significantly higher levels in saturated fatty acids in response to Western-type diet (Supplementary Table 4). More importantly, there was an increase in monounsaturated fatty acids and decrease in polyunsaturated fatty acids mainly in response to Western-type diet and to a lesser degree to ApoE deficiency (Supplementary Table 4). Taken together, these findings indicate that ApoE deficiency and Western-type diet independently increased intramuscular fat accumulation without any synergistic effects.

Given this remarkable accumulation of intramuscular fat and considering the increasing body of evidence that oxidised phospholipids are key players in the development of chronic inflammatory disease and potentially atherosclerosis, we next focused on skeletal muscle phospholipids [43]. Quantification of total phospholipids in skeletal muscle specimens did not 
reveal any significant differences in response to either ApoE deficiency or dietary challenges, despite significant effects on some individual fatty acids (Supplementary Fig. 3C and Supplementary Table 5). We next determined whether there were oxidised phospholipids in skeletal muscle specimens. Increased levels of oxidised phospholipids were found in $\mathrm{ApoE}^{-/}$WD muscles compared to all other experimental conditions (Supplementary Fig. 4A). There was a co-localisation of oxidised phospholipid staining with CD31 staining that identifies muscle capillaries, suggesting that the oxidised phospholipids were probably from circulating oxidised LDL (Supplementary Fig. 4B).

Metabonomics: Muscle metabolite profiles were determined by ${ }^{1} \mathrm{H}$ nuclear magnetic resonance spectroscopy. The scores plot from the PCA model revealed distinct metabolic signatures among experimental groups (Fig. 4A). WT WD and $A p o E^{---}$ND mice appeared to have common metabolic profiles however, the combination of genotypic and dietary differences in the WT ND and $A p o E^{-/-}$WD mice resulted in remarkable metabolic differences in the muscles of these mice. Unsupervised hierarchical clustering analysis of the metabolic variation that was associated with the different genotypes and dietary regimes is presented in Figure 4B. WT ND and $A p o E^{---}$WD mice form the most distinct clusters in the clustergram whereas WT WD and $\mathrm{ApoE}^{-/-}$ND share a great degree of metabolic similarity. Metabolic differences originate in increases in taurine, creatine and lysine in $\mathrm{ApoE}^{-/-}$WD (Fig. 4B) and concomitant decreases in branched chain amino acids (valine, leucine, isoleucine), choline metabolites (dimethylglycine, TMA), 3- and 4- hydroxybutyrate, intermediates of oxidative metabolism (succinate, malate, citrate) and products of glycolytic metabolism (lactate). Decreases in anserine, AMP, malonate, glycerol, NAD+, tyrosine, aspartate, glucose and acetate were also observed in the muscles of $\mathrm{ApoE}^{-/-}$mice fed a Western-type diet.

The impact of Western-type diet and ApoE deficiency in skeletal muscle oxidative stress 
Ectopic lipid accumulation is linked to increased oxidative stress and pathogenesis [44, 45]. We found significantly higher mRNA levels of Nox2, Tnfa and $/ / 1 b$ - involved in oxidative stress and inflammation - in gastrocnemius muscle of all experimental groups compared to WT ND (Fig. 5A). There were significantly lower mRNA levels for a number of known antioxidant genes; glutathione peroxidase 1 (Gpx1), glutathione peroxidase 4 (Gpx4), peroxiredoxin $1(\operatorname{Prd} 1)$, superoxide dismutase 1 (Sod1) and microsomal Glutathione STransferase 1 (Mgst1) in response to diet (Gpx1, Gpx4, Prdx1 and Mgst1) and ApoE deficiency (Gpx4, Prdx1, and Mgst1) (Fig. 5A). Catalase mRNA levels were however over $400 \%$ upregulated in response to either ApoE deficiency or diet (Fig. 5A). Transcript levels of two DNA repair enzymes [Poly (ADP-ribose) polymerase 1 (Parp1) and 8-Oxoguanine glycosylase (Ogg1)] were significantly higher in all three groups (WT WD, ApoE ${ }^{-/-}$ND and ApoE $^{-/-}$WD) compared to WT ND (Fig. 5A). These findings suggest perturbations in genes regulating oxidative stress, inflammation, antioxidant properties and DNA repair of the skeletal muscle in response to ApoE deficiency and Western-type diet without any interaction between these two factors.

Dihydroethidium (DHE) staining as a read out of free radicals revealed significantly higher levels in response to ApoE deficiency or Western-type diet (Fig. 5B). Superoxide quantification by LC-MS showed higher levels in response to Western-type diet (Supplementary Fig. 5A). Specimen treatment with the free-radical scavenger polyethylene glycol-superoxide dismutase (PEG-SOD) revealed, as expected, no DHE staining indicative of staining specificity (Supplementary Fig. 5B). Consistent with the finding of increased free radicals, significantly higher levels of protein carbonyls and tyrosine nitration, as well as lipid peroxidation by means of TBARS and 4-hydroxy-2-nonenal (4HNE) adducts were found in the quadriceps and TA of WT WD, ApoE ${ }^{-/-}$ND and $A p o E^{--}$WD as compared to WT ND (Fig. 5C-E, Supplementary Fig. 5C-E).

Evidence suggests that increased ROS production in the aorta is accompanied by upregulation of extracellular signal-regulated protein kinases 1 and 2 (ERK1/2) and p38 
mitogen-activated protein kinases (MAPKs) in response to high-fat diet as well as atherosclerosis [46, 47]. Therefore, we determined the phosphorylation levels of various MAPKs in the skeletal muscle using a phospho-MAPK array kit [48]. We found that phosphorylation levels of ERK1 and ERK2 were increased in response to genotype and diet (Supplementary Figure 6A). Phosphorylation levels of $p 38 \alpha, y$ and $\delta$ were increased only in the WT WD group (Supplementary Figure 6A). To validate the findings for p38, immunoblotting for phospho- and total levels of p38 was conducted. Indeed, phosphorylation of p38 MAPK was increased only in the WT WD group (Supplementary Figure 6B). Furthermore, phosphorylation levels of protein kinase B (Akt) isoforms, total Akt, Glycogen synthase kinase-3 alpha and beta $(G s k 3 \alpha / \beta)$ as well as cAMP response element binding protein (CREB) were upregulated in response to both ApoE deficiency and western-type diet. MKK6 phosphorylation was increased two-fold by western-type diet in both genotypes (Supplementary Figure 6A).

Staining of muscle specimens with an antibody against 8-OHdG, an oxidised derivative of deoxyguanosine, showed increased fluorescence in WT WD and $A p o E^{-/-}$WD muscles compared to WT ND (Supplementary Fig. 7). Given the increased transcript levels of Tnfa in all groups compared to WT ND, we also studied macrophage infiltration in skeletal muscle by conducting F4.80 staining. There was no evidence of macrophage infiltration in skeletal muscle in any of the experimental groups (Supplementary Figure 8). Taken together, these findings show that ApoE deficiency and Western-type diet independently increase oxidative stress in skeletal muscles originating in high ROS, protein carbonylation, tyrosine nitration, lipid peroxidation and DNA damage.

\section{Western-type diet and ApoE deficiency promote the development of liver steatosis, inflammation and oxidative stress}

Given the robust data of increased oxidative stress in skeletal muscle of mice subjected to either Western-type diet or ApoE deficiency and increased intramuscular lipid accumulation, 
we next sought to establish whether there was evidence of steatosis, inflammation or oxidative stress in another metabolically active organ, the liver. Oil Red O staining provided strong evidence of liver steatosis in response to Western-type diet and ApoE deficiency, while fat droplets were largest in $\mathrm{ApoE}^{-/-} \mathrm{WD}$, revealing a synergistic effect of ApoE deficiency and dietary challenge (Supplementary Fig. 9A). We also found evidence of inflammation by increased macrophage accumulation in the liver in response to both diet and ApoE deficiency (Supplementary Fig. 9B). Transcript levels of genes regulating fatty acid uptake and oxidation were significantly higher in response to both ApoE deficiency and diet (Cd36, Stearoyl-CoA desaturase; Scd1) or in response to ApoE deficiency only (Hydroxyacyl-CoA dehydrogenase; Had and Lipoprotein lipase; Lpl) (Supplementary Fig. 9C). There were large inter-individual differences within experimental groups for inflammatory genes (Tnfa, Mcp1 and F4.80) and significantly higher levels were evident only in wild-type mice subjected to Western-type diet (Tnfa, Mcp1) (Supplementary Fig. 9C).

DHE staining showed increased fluorescence in $A p o E^{-/}$WD compared to WT ND (Supplementary Fig. 10A). Conversely, mRNA levels of antioxidant genes (Gpx1, Gpx4, Sod1, Sod2 and Prdx1), lipid peroxidation levels, assessed by means of TBARS, and protein carbonyls were not significantly altered by diet or genotype (Supplementary Fig. 10B-C). However, there was evidence of significantly higher tyrosine nitration in ApoE deficient mice independent of their dietary status for a protein at 25kDa (Supplementary Fig. 10D). Lastly, 4HNE adducts in histidine residues were higher in response to both ApoE deficiency and Western-type diet (Supplementary Fig. 10E).

The impact of NADPH oxidase 2 inhibition on levels of oxidative stress in skeletal muscle and the liver of ApoE deficient mice

NADPH oxidases are known to be key players in ROS production and induction of cellular oxidative stress [20]. Nox2 inhibition has been employed as a tool to reduce oxidative stress in the aorta of atherosclerotic mice $[21,31]$. We determined the impact of Nox2 
pharmacological inhibition by means of the Nox2ds-tat peptide on the levels of oxidative stress seen in $\mathrm{ApoE}^{-/-}$mice exposed to a Western-type diet (Fig. 6A). DHE staining was significantly lower in skeletal muscle of Nox2ds-tat treated mice compared to scrambled peptide (Fig. 6B). There was no effect on the mRNA levels of antioxidant genes (data not shown), but we found significantly lower mRNA levels of Parp1 (Fig. 6C). We next examined the effect of Nox2 inhibition on oxidative protein modifications in two skeletal muscle beds. Nox2 inhibition resulted in significantly lower levels of protein carbonylation in the quadriceps and tibialis anterior without any effect on tyrosine nitration or 4HNE adducts in histidine residues (Fig. 6D and Supplementary Fig. 11A). Interestingly, we found reduced 8-OHdG immunofluorescence (Supplementary Fig. 11B), in the absence of any other differences in response to Nox2ds-tat treatment in terms of body weight, fibre type, SDH activity, capillary density in EDL and TA muscles and intramuscular triacylglycerol contents (Supplementary Fig. 12A-E).

We also sought to determine whether treatment with Nox2ds-tat peptide revealed no differences in the levels of steatosis and macrophage accumulation in the liver (Fig. 7A-B). Except for a decrease of the DHE staining there was no significant difference in oxidative protein modifications in response to Nox2 inhibition (Fig. 7C-E). These findings suggest that Nox2 inhibition did not exhibit any major effect on oxidative stress, steatosis and inflammation in the liver. Therefore, we propose here that ApoE deficiency combined with an atherogenic diet leads to hyperlipidaemia, ectopic fat accumulation and oxidative stress. In turn, Nox2 inhibition can mitigate oxidative stress in skeletal muscle but not the liver.

\section{Discussion}

The obesity-independent impact of hyperlipidaemia and atherosclerosis has only recently gained scientific attention [6, 49]. Thus, it is unclear whether hyperlipidaemia and atherosclerosis affect skeletal muscle biology in the absence of obesity, since, in most studies, obesity and other comorbidities are present $[50,51]$. Here, we sought to determine 
for the first time whether hyperlipidaemia and atherosclerosis impact on skeletal muscle metabolic and redox homeostasis in the absence of obesity. The differential effect of western-type diet on body mass of $\mathrm{ApoE}^{-/-}$and WT mice reported here appears to be in contrast with a previous study where $\mathrm{ApoE}^{-/-}$mice gained more body weight compared to WT mice on a western-type diet [52]. However, our findings are in line with several previous studies showing that $\mathrm{ApoE}^{-/-}$mice gain less body weight on a western-type or high-fat diet as compared to wild-type mice $[16,18,53,54]$. In addition, ApoE deficiency on an obese background shows a milder weight gain as compared to obese mice [55, 56]. This may possibly be explained by both lower synthesis and increased hydrolysis of triacylglycerols from ApoE $^{-/}$adipocytes $[16,18,57]$.

Expression of specific myosin isoforms is considered as a major determinant of both contractile and metabolic properties which, in turn, are controlled by distinct signalling pathways [40]. Of note, the effect of a high-fat diet supplemented with cholesterol on skeletal muscle morphology has not been investigated so far. We have shown that ApoE deficiency results in a partial fibre type transition from IIB to IIX independent of dietary challenge. From a biological point of view this may represent a functional adaptation to a hyperlipidaemic environment, where the fast-twitch glycolytic IIB fibres are not able to obtain energy from fat in contrast to fast-twitch IIX fibres that can rely on oxidative metabolism [40]. Western-type diet in wild-type mice induced a transition to more oxidative myofibres only in the soleus. Numerous studies have investigated the effect of high-fat diet on skeletal myofibre composition of wild-type mice, with contrasting results [58-62]. This discrepancy may be accounted for by differences in diet composition (i.e. percentage of total energy from fat and type of fatty acids) and the particular muscle beds examined [63].

In turn, coordinated changes between myofibre type and mitochondrial oxidative capacity are not uncommon under physiological conditions [64]. However, the present study has shown that under hyperlipidaemic and atherosclerotic conditions, a transition to slower myofibres is not accompanied by a concomitant increase in mitochondrial activity, possibly 
due to metabolic perturbations or mitochondrial dysfunction (as shown below by metabonomics).

Capillaries supply myofibres with oxygen and nutrients and their number may change in response to altered oxygen and nutrient demands of the myofibre [40]. ApoE deficiency and Western-type diet independently induced a higher capillary-to-fibre-ratio in the glycolytic EDL and superficial TA, followed by increased mRNA levels of key angiogenic factors. These findings may be explained by recent data suggesting that local capillarisation is determined by the size and metabolic products of the myofibre $[65,66]$.

Indeed, myofibre cross-sectional area was larger in response to ApoE deficiency and hypertrophic myofibres were observed in all muscle beds studied independently of the individual muscle metabolic phenotype. ApoE deficiency, combined with diet had a synergistic effect on myofibre size (Fig. 1D). Changes in myofibre size were accompanied by increased mRNA levels of the major skeletal muscle fatty acid transporters and intramuscular lipid accumulation, indicating that ectopic lipid accumulation and/or myofibre transition towards slower types may both account for the enlarged myofibres. Surprisingly, the increase in plasma membrane-bound CD36 was greater in wild-type mice on Westerntype diet than in $\mathrm{ApoE}^{-/-}$mice irrespective of the administered diet. This may be explained by the absence of previous high-fat exposure of cells in wild-type mice, which resulted in stronger upregulation of $\mathrm{Cd} 36$ than in cells of $\mathrm{ApoE}^{-/-}$mice which had been exposed to high plasma levels of atherogenic lipids since early life. In support of this notion, $\mathrm{ApoE}^{-/-}$mice exhibited 100\% higher in CD36 gene and protein expression in the heart at the age of 6 weeks compared to wild-type mice, whereas at the age of 20 weeks, CD36 gene and protein expression were only $30 \%$ and $40 \%$ higher respectively [67]. Another possible explanation is that ApoE deficiency affects CD36 gene and protein expression in skeletal muscle indirectly. However, further research is needed to elucidate the exact mechanisms, since ApoE is present not only in capillaries but also in skeletal muscle stem cells [68, 69]. Gas chromatography for triacylglycerol fatty acid composition revealed significantly higher levels 
in saturated fatty acids mainly in the Western-type diet groups. This finding recapitulates unfavourable intramuscular lipid changes attributed mainly to the dietary challenge as described previously $[63,70]$.

Absence of striking changes in phospholipid fatty acid composition was followed by investigation of the abundance of oxidised phospholipids. Oxidised phospholipids are key players in the development of chronic inflammatory disease [43]. Oxidised phospholipids were found mostly in $\mathrm{ApoE}^{-/-}$WD muscles and they were localised in muscle capillaries. It has been shown that $A p o E^{-/-}$mice have a transient increase in plasma oxidised LDL at the age of 20 weeks old, which coincides with the age of the mice of the present study [71]. This may explain the high abundance of oxidised phospholipids in ApoE muscles given that $\mathrm{ApoE}^{-/-}$mice have oxidised LDL that is exacerbated by Western-type diet.

Considering the intramuscular lipid accumulation and concomitant positive myofibre remodelling, we investigated the metabolic signature of skeletal muscle. Previous studies in wild-type mice on a high-fat diet have shown that accumulation of intramyocellular lipids leads to increase, decrease or no effect on fatty acid oxidation and mitochondrial respiration [61, 72-75]. This lack of agreement may be attributed to factors, such as age of the animals at diet commencement, sex and strain of mice, duration and composition of diet. Metabonomic analysis unveiled a reduction of lactate, metabolites of tricarboxylic acid cycle (malate, citrate and succinate) and branched-chain amino acids in response to either ApoE deficiency or Western-type diet. Importantly, ApoE deficiency combined with atherogenic diet had the most profound impact on skeletal muscle metabolites. Thus, it can be assumed that there may be a shift in energy production at the expense of anaerobic carbohydrate breakdown (i.e. lactate), indicating skeletal muscle metabolic perturbations. Furthermore, the creatine increase in response to both ApoE deficiency and Western-type diet may be linked to a previously reported antioxidant effect [76]. Taurine, a peptide implicated in antioxidant defence and stress responses, was increased in response to either ApoE deficiency or Western-type diet and was greatest in the $\operatorname{ApoE}^{-/-}$WD group $[33,77]$. This increase may be 
useful to scavenge products of oxidation due to lipid accumulation. On the other hand, anserine, a peptide also involved in antioxidant defence was increased in response to Western-type diet in wild-type mice and ApoE deficiency but was decreased in $\mathrm{ApoE}^{-/-}$mice when administered a Western-type diet, possibly due to impaired redox homeostasis [33, 37]. Taken together, our findings indicate that the transition to slower myofibres and enhanced capillarisation caused by ApoE deficiency and Western-type diet, possibly as a compensation for the excess fat, were not adequate to increase the utilisation of the excess fat, as shown by decreased metabolites of the tricarboxylic acid cycle and subtle effects on SDH activity, suggesting that there may be impaired fatty acid $\beta$ oxidation.

Similarly, ectopic fat accumulation was evident in the liver in line with previous studies [18, $78,79]$. Steatosis was accompanied by increased mRNA levels of genes involved in fatty acid uptake and oxidation as well as hepatic inflammation that was not aggravated by Western-type diet. This finding suggests that ApoE deficiency alone is a major contributor in development of inflammation independent of diet as reported previously [78]. Overall, our data support the notion that hyperlipidaemia is the main factor for the development of hepatic steatosis and inflammation and a diet supplemented with cholesterol exacerbates steatosis and increases genes involved in fatty acid metabolism.

Ectopic fat accumulation has been associated with redox imbalance in skeletal muscle of wild-type mice on high-fat diet $[51,80]$. We provide robust evidence that ApoE deficiency and Western-type diet separately induce skeletal muscle oxidative stress as shown by increased ROS production, perturbed antioxidant gene expression and elevated oxidative protein modifications in quadriceps and tibialis anterior. This finding is expanded by evidence on increased DNA damage in response to Western-type diet and elevated mRNA levels of DNA-repair enzymes in response to either ApoE deficiency or Western-type diet. Consequently, it can be concluded that hyperlipidaemia and atherosclerosis induce oxidative stress in skeletal muscle. However, the diet appears to have a more profound effect on DNA damage. Conversely, increased oxidative modifications of hepatic proteins appear to depend 
more on the genotype. This finding is in agreement with previous studies that reported increased markers of hepatic oxidative stress in ApoE deficient mice compared to wild-type mice $[13,78]$.

In this study, phosphorylation of ERK1/2 and CREB was upregulated by ApoE deficiency and western-type diet, whereas phosphorylation of $p 38 \alpha$ and $y$ was upregulated by westerntype diet only in the wild type mice. MAPKs are important regulators of various cellular functions, such as proliferation, differentiation and survival [81]. ERK and p38, are known to be activated by oxidative stress and Nox2 has been previously shown to upregulate vascular MAPKs in rats administered high-fat diet [46, 82]. Similarly, free fatty acids induce upregulation of the above MAPKs both in C2C12 cells and high-fat diet in humans [83, 84]. Collectively, these data indicate that the effects of western-type diet can, at least in part, be mediated by MAPKs (ERK1/2, p38 and CREB) highlighting their potential role in adult skeletal muscle maintenance $[85,86]$.

Surprisingly, there was not any did not have a cumulative effect of western-type diet on skeletal muscle of $\mathrm{ApoE}^{-/-}$mice for most parameters studied apart from metabonomics and triacylglycerol species. However, these findings are in line with previous studies that reported similar findings on skeletal muscle of $\mathrm{ApoE}^{-/-}$mice on either a standard chow or high-fat diet $[13,16]$. In both studies, high-fat diet in $\mathrm{ApoE}^{-/-}$mice failed to induce any cumulative effect on skeletal muscle markers of oxidative stress and inflammation. We showed that $\mathrm{ApoE}^{-l-}$ mice under basal conditions (i.e. chow diet) have enhanced fat contents but greater lipid accumulation in skeletal muscle under WD was not evident. This assumption is further supported by the unaltered expression of CD36 in $\mathrm{ApoE}^{-/-}$mice on a WD compared to $\mathrm{ApoE}^{-/-}$mice on a chow diet. Further investigation is needed to gain a mechanistic insight on how western-type diet and ApoE deficiency affect cholesterol accumulation in skeletal muscle.

Based on the studies that show an important role of Nox2 in ROS production, we examined whether Nox2 inhibition would attenuate oxidative stress in skeletal muscle and protein 
modifications in the liver $[24,87,88]$. Consistent with our hypothesis, Nox2 inhibition had a striking effect on oxidative stress in skeletal muscle by reducing superoxide production and protein carbonylation. Protein 4HNE adducts and tyrosine nitration were not altered, suggesting that other enzymes contribute to oxidative stress. Transcriptional levels of the proinflammatory cytokine Tnfa were also decreased. Therefore, Nox2 is a major contributor of ROS production and oxidative damage in skeletal muscle of $\mathrm{ApoE}^{-{ }^{--}}$mice. Nox2 inhibition had no impact on myofibre composition which is to be expected given the duration of the treatment and the low turn-over rate of MHC. Moreover, Nox2 inhibition did not appear to impact on hepatic steatosis, inflammation or oxidative protein modifications in the liver. However, it cannot be ruled out that the duration of treatment in the present study may have not been sufficient to induce changes in hepatic physiology. It can be assumed that the lack of changes in the liver of $\mathrm{ApoE}^{-/-}$mice treated with the Nox2ds-tat inhibitor may be attributed to: i) the duration of treatment or the chosen dosage not being sufficient to induce changes in the liver or ii) Nox2 not being a prominent source of hepatic oxidative modifications in ApoE $^{-/-}$mice.

\section{Conclusions}

This is the first study to investigate in depth the obesity-independent effect of hyperlipidaemia and atherosclerosis on skeletal muscle biology. Hyperlipidaemia and atherosclerosis, independent of obesity, induced intramuscular lipid accumulation. Skeletal muscle, as a highly adaptive tissue, responded with enhanced capillarisation and a transition to more oxidative myofibres. However, this adaptation was not sufficient to overcome the lipid accumulation that, at least partially, led to decreased mitochondrial metabolites and increased oxidative stress. Treatment with a potential Nox2 inhibitor resulted in attenuation of muscle oxidative stress, demonstrating that Nox2 is an important contributor to skeletal muscle oxidative stress under hyperlipidaemic conditions. Our results have translational implications for the use of muscle-targeted treatment to attenuate oxidative stress. 


\section{Declarations}

\section{Availability of data and materials}

No additional data are available.

\section{Declarations of interest}

The authors declare that they have no competing interests.

\section{Funding}

The study was supported by BHF Project Grant - PG/14/54/30939 and Programme Grant RG/09/010/28087 to MTK. The study was also supported by the European Union and The Royal Society (Grants: FP7-PEOPLE-PCIG14-GA-2013-631440 and RG140470 Research Grant to AM).

\section{Acknowledgements}

We are thankful to Mr Andrew Gordon for technical support with the LC-MS experiments. 


\section{References}

[1] R.H. Nelson, Hyperlipidemia as a risk factor for cardiovascular disease, Prim Care 40(1) (2013) 195-211.

[2] U.J. Jung, M.S. Choi, Obesity and its metabolic complications: the role of adipokines and the relationship between obesity, inflammation, insulin resistance, dyslipidemia and nonalcoholic fatty liver disease, Int J Mol Sci 15(4) (2014) 6184-223.

[3] R. Poledne, I. Kralova Lesna, S. Cejkova, Adipose tissue and atherosclerosis, Physiological research 64 Suppl 3 (2015) S395-402.

[4] X. Xu, L. Lu, Q. Dong, X. Li, N. Zhang, Y. Xin, S. Xuan, Research advances in the relationship between nonalcoholic fatty liver disease and atherosclerosis, Lipids Health Dis 14 (2015) 158.

[5] E. Gaudio, V. Nobili, A. Franchitto, P. Onori, G. Carpino, Nonalcoholic fatty liver disease and atherosclerosis, Internal and emergency medicine 7 Suppl 3 (2012) S297-305.

[6] D. Kim, W.R. Kim, Nonobese Fatty Liver Disease, Clinical gastroenterology and hepatology : the official clinical practice journal of the American Gastroenterological Association 15(4) (2017) 474-485.

[7] A. Hernandez-Aguilera, J. Sepulveda, E. Rodriguez-Gallego, M. Guirro, A. GarciaHeredia, N. Cabre, F. Luciano-Mateo, I. Fort-Gallifa, V. Martin-Paredero, J. Joven, J. Camps, Immunohistochemical analysis of paraoxonases and chemokines in arteries of patients with peripheral artery disease, Int J Mol Sci 16(5) (2015) 11323-38.

[8] G.I. Shulman, Ectopic fat in insulin resistance, dyslipidemia, and cardiometabolic disease, The New England journal of medicine 371(12) (2014) 1131-41.

[9] T.L. Haas, P.G. Lloyd, H.T. Yang, R.L. Terjung, Exercise training and peripheral arterial disease, Comprehensive Physiology 2(4) (2012) 2933-3017.

[10] K. Cluff, D. Miserlis, G.K. Naganathan, Pipinos, II, P. Koutakis, A. Samal, R.D. McComb, J. Subbiah, G.P. Casale, Morphometric analysis of gastrocnemius muscle biopsies from patients with peripheral arterial disease: objective grading of muscle degeneration, Am J Physiol Regul Integr Comp Physiol 305(3) (2013) R291-9. 
[11] P. Koutakis, D. Miserlis, S.A. Myers, J.K. Kim, Z. Zhu, E. Papoutsi, S.A. Swanson, G. Haynatzki, D.M. Ha, L.A. Carpenter, R.D. McComb, J.M. Johanning, G.P. Casale, Pipinos, II, Abnormal accumulation of desmin in gastrocnemius myofibers of patients with peripheral artery disease: associations with altered myofiber morphology and density, mitochondrial dysfunction and impaired limb function, J Histochem Cytochem 63(4) (2015) 256-69.

[12] Pipinos, II, A.R. Judge, J.T. Selsby, Z. Zhu, S.A. Swanson, A.A. Nella, S.L. Dodd, The myopathy of peripheral arterial occlusive disease: part 1. Functional and histomorphological changes and evidence for mitochondrial dysfunction, Vasc Endovascular Surg 41(6) (2007) 481-9.

[13] S.S. Pereira, L.G. Teixeira, E.C. Aguilar, R.O. Matoso, F.L. Soares, A.V. Ferreira, J.I. Alvarez-Leite, Differences in adipose tissue inflammation and oxidative status in C57BL/6 and ApoE-/- mice fed high fat diet, Anim Sci J 83(7) (2012) 549-55.

[14] P.A. Stapleton, A.G. Goodwill, M.E. James, A.C. D'Audiffret, J.C. Frisbee, Differential impact of familial hypercholesterolemia and combined hyperlipidemia on vascular wall and network remodeling in mice, Microcirculation 17(1) (2010) 47-58.

[15] P.A. Stapleton, A.G. Goodwill, M.E. James, J.C. Frisbee, Altered mechanisms of endothelium-dependent dilation in skeletal muscle arterioles with genetic hypercholesterolemia, Am J Physiol Regul Integr Comp Physiol 293(3) (2007) R1110-9.

[16] J. Wang, X.D. Perrard, J.L. Perrard, A. Mukherjee, C. Rosales, Y. Chen, C.W. Smith, H.J. Pownall, C.M. Ballantyne, H. Wu, ApoE and the role of very low density lipoproteins in adipose tissue inflammation, Atherosclerosis 223(2) (2012) 342-9.

[17] P. Sfyri, A. Matsakas, Crossroads between peripheral atherosclerosis, western-type diet and skeletal muscle pathophysiology: emphasis on apolipoprotein E deficiency and peripheral arterial disease, Journal of biomedical science 24(1) (2017) 42.

[18] A. Bartelt, P. Orlando, C. Mele, A. Ligresti, K. Toedter, L. Scheja, J. Heeren, V. Di Marzo, Altered endocannabinoid signalling after a high-fat diet in Apoe(-/-) mice: 
relevance to adipose tissue inflammation, hepatic steatosis and insulin resistance, Diabetologia 54(11) (2011) 2900-10.

[19] R.P. Brandes, N. Weissmann, K. Schroder, Redox-mediated signal transduction by cardiovascular Nox NADPH oxidases, Journal of molecular and cellular cardiology 73 (2014) 70-9.

[20] G.R. Drummond, C.G. Sobey, Endothelial NADPH oxidases: which NOX to target in vascular disease?, Trends Endocrinol Metab 25(9) (2014) 452-63.

[21] C.P. Judkins, H. Diep, B.R. Broughton, A.E. Mast, E.U. Hooker, A.A. Miller, S. Selemidis, G.J. Dusting, C.G. Sobey, G.R. Drummond, Direct evidence of a role for Nox2 in superoxide production, reduced nitric oxide bioavailability, and early atherosclerotic plaque formation in ApoE-/- mice, American journal of physiology. Heart and circulatory physiology 298(1) (2010) H24-32.

[22] L.F. Ferreira, O. Laitano, Regulation of NADPH oxidases in skeletal muscle, Free Radic Biol Med 98 (2016) 18-28.

[23] A. Espinosa, C. Campos, A. Diaz-Vegas, J.E. Galgani, N. Juretic, C. Osorio-Fuentealba, J.L. Bucarey, G. Tapia, R. Valenzuela, A. Contreras-Ferrat, P. Llanos, E. Jaimovich, Insulin-dependent $\mathrm{H} 2 \mathrm{O} 2$ production is higher in muscle fibers of mice fed with a high-fat diet, Int J Mol Sci 14(8) (2013) 15740-54.

[24] A. Souto Padron de Figueiredo, A.B. Salmon, F. Bruno, F. Jimenez, H.G. Martinez, G.V. Halade, S.S. Ahuja, R.A. Clark, R.A. DeFronzo, H.E. Abboud, A. El Jamali, Nox2 mediates skeletal muscle insulin resistance induced by a high fat diet, J Biol Chem 290(21) (2015) 13427-39.

[25] T. Yokota, S. Kinugawa, K. Hirabayashi, S. Matsushima, N. Inoue, Y. Ohta, S. Hamaguchi, M.A. Sobirin, T. Ono, T. Suga, S. Kuroda, S. Tanaka, F. Terasaki, K. Okita, H. Tsutsui, Oxidative stress in skeletal muscle impairs mitochondrial respiration and limits exercise capacity in type 2 diabetic mice, American journal of physiology. Heart and circulatory physiology 297(3) (2009) H1069-77. 
[26] M.E. Cifuentes-Pagano, D.N. Meijles, P.J. Pagano, Nox Inhibitors \& Therapies: Rational Design of Peptidic and Small Molecule Inhibitors, Curr Pharm Des 21(41) (2015) 602335.

[27] P. Sukumar, H. Viswambharan, H. Imrie, R.M. Cubbon, N. Yuldasheva, M. Gage, S. Galloway, A. Skromna, P. Kandavelu, C.X. Santos, V.K. Gatenby, J. Smith, D.J. Beech, S.B. Wheatcroft, K.M. Channon, A.M. Shah, M.T. Kearney, Nox2 NADPH oxidase has a critical role in insulin resistance-related endothelial cell dysfunction, Diabetes 62(6) (2013) 2130-4.

[28] H. Viswambharan, N.Y. Yuldasheva, A. Sengupta, H. Imrie, M.C. Gage, N. Haywood, A.M. Walker, A. Skromna, N. Makova, S. Galloway, P. Shah, P. Sukumar, K.E. Porter, P.J. Grant, A.M. Shah, C.X. Santos, J. Li, D.J. Beech, S.B. Wheatcroft, R.M. Cubbon, M.T. Kearney, Selective Enhancement of Insulin Sensitivity in the Endothelium In Vivo Reveals a Novel Proatherosclerotic Signaling Loop, Circulation research 120(5) (2017) 784-798.

[29] X. Zhou, B. Teng, S.J. Mustafa, Sex Difference in Coronary Endothelial Dysfunction in Apolipoprotein E Knockout Mouse: Role of NO and A2A Adenosine Receptor, Microcirculation 22(7) (2015) 518-27.

[30] M.R. DiStasi, J.A. Mund, H.G. Bohlen, S.J. Miller, D.A. Ingram, M.C. Dalsing, J.L. Unthank, Impaired compensation to femoral artery ligation in diet-induced obese mice is primarily mediated via suppression of collateral growth by Nox2 and p47phox, American journal of physiology. Heart and circulatory physiology 309(7) (2015) H1207-17.

[31] I.M. Quesada, A. Lucero, C. Amaya, D.N. Meijles, M.E. Cifuentes, P.J. Pagano, C. Castro, Selective inactivation of NADPH oxidase 2 causes regression of vascularization and the size and stability of atherosclerotic plaques, Atherosclerosis 242(2) (2015) 46975.

[32] S. Magwenzi, C. Woodward, K.S. Wraith, A. Aburima, Z. Raslan, H. Jones, C. McNeil, S. Wheatcroft, N. Yuldasheva, M. Febbriao, M. Kearney, K.M. Naseem, Oxidized LDL 
activates blood platelets through CD36/NOX2-mediated inhibition of the cGMP/protein kinase G signaling cascade, Blood 125(17) (2015) 2693-703.

[33] A. Matsakas, D.A. Prosdocimo, R. Mitchell, H. Collins-Hooper, N. Giallourou, J.R. Swann, P. Potter, T. Epting, M.K. Jain, K. Patel, Investigating mechanisms underpinning the detrimental impact of a high-fat diet in the developing and adult hypermuscular myostatin null mouse, Skeletal muscle 5 (2015) 38.

[34] A. Matsakas, V. Yadav, S. Lorca, V. Narkar, Muscle ERRgamma mitigates Duchenne muscular dystrophy via metabolic and angiogenic reprogramming, FASEB journal : official publication of the Federation of American Societies for Experimental Biology 27(10) (2013) 4004-16.

[35] V. Mougios, A. Petridou, Analysis of lipid profiles in skeletal muscles, Methods in molecular biology 798 (2012) 325-55.

[36] O. Beckonert, H.C. Keun, T.M. Ebbels, J. Bundy, E. Holmes, J.C. Lindon, J.K. Nicholson, Metabolic profiling, metabolomic and metabonomic procedures for NMR spectroscopy of urine, plasma, serum and tissue extracts, Nature protocols 2(11) (2007) 2692-703.

[37] S. Omairi, A. Matsakas, H. Degens, O. Kretz, K.A. Hansson, A.V. Solbra, J.C. Bruusgaard, B. Joch, R. Sartori, N. Giallourou, R. Mitchell, H. Collins-Hooper, K. Foster, A. Pasternack, O. Ritvos, M. Sandri, V. Narkar, J.R. Swann, T.B. Huber, K. Patel, Enhanced exercise and regenerative capacity in a mouse model that violates size constraints of oxidative muscle fibres, eLife 5 (2016).

[38] C. Kilkenny, W.J. Browne, I.C. Cuthill, M. Emerson, D.G. Altman, Improving bioscience research reporting: the ARRIVE guidelines for reporting animal research, PLoS biology 8(6) (2010) e1000412.

[39] F. Faul, E. Erdfelder, A.G. Lang, A. Buchner, G*Power 3: a flexible statistical power analysis program for the social, behavioral, and biomedical sciences, Behavior research methods 39(2) (2007) 175-91. 
[40] S. Schiaffino, C. Reggiani, Fiber types in mammalian skeletal muscles, Physiol Rev 91(4) (2011) 1447-531.

[41] Y. Pincu, M.A. Linden, K. Zou, T. Baynard, M.D. Boppart, The effects of high fat diet and moderate exercise on TGFbeta1 and collagen deposition in mouse skeletal muscle, Cytokine 73(1) (2015) 23-9.

[42] C.S. Tam, J.E. Power, T.P. Markovic, C. Yee, M. Morsch, S.V. McLennan, S.M. Twigg, The effects of high-fat feeding on physical function and skeletal muscle extracellular matrix, Nutr Diabetes 5 (2015) e187.

[43] V. Bochkov, B. Gesslbauer, C. Mauerhofer, M. Philippova, P. Erne, O.V. Oskolkova, Pleiotropic effects of oxidized phospholipids, Free Radic Biol Med 111 (2017) 6-24.

[44] J. Boren, M.R. Taskinen, S.O. Olofsson, M. Levin, Ectopic lipid storage and insulin resistance: a harmful relationship, Journal of internal medicine 274(1) (2013) 25-40.

[45] D.L. Johannsen, K.E. Conley, S. Bajpeyi, M. Punyanitya, D. Gallagher, Z. Zhang, J. Covington, S.R. Smith, E. Ravussin, Ectopic lipid accumulation and reduced glucose tolerance in elderly adults are accompanied by altered skeletal muscle mitochondrial activity, The Journal of clinical endocrinology and metabolism 97(1) (2012) 242-50.

[46] S. Touati, A.C. Montezano, F. Meziri, C. Riva, R.M. Touyz, P. Laurant, Exercise training protects against atherosclerotic risk factors through vascular NADPH oxidase, extracellular signal-regulated kinase $1 / 2$ and stress-activated protein kinase/c-Jun $\mathrm{N}$ terminal kinase downregulation in obese rats, Clinical and experimental pharmacology \& physiology 42(2) (2015) 179-85.

[47] Y. Wu, F. Wang, L. Fan, W. Zhang, T. Wang, Y. Du, X. Bai, Baicalin alleviates atherosclerosis by relieving oxidative stress and inflammatory responses via inactivating the NF-kappaB and p38 MAPK signaling pathways, Biomedicine \& pharmacotherapy $=$ Biomedecine \& pharmacotherapie 97 (2018) 1673-1679.

[48] A. Fullar, K. Baghy, F. Deak, B. Peterfia, Y. Zsak, P. Tatrai, Z. Schaff, J. Dudas, I. Kiss, I. Kovalszky, Lack of Matrilin-2 favors liver tumor development via Erk1/2 and GSK-3beta pathways in vivo, PloS one 9(4) (2014) e93469. 
[49] S.D. Moscavitch, H.C. Kang, R.A. Filho, E.T. Mesquita, H.C. Neto, M.L. Rosa, Comparison of adipokines in a cross-sectional study with healthy overweight, insulinsensitive and healthy lean, insulin-resistant subjects, assisted by a family doctor primary care program, Diabetology \& metabolic syndrome 8 (2016) 9.

[50] I.M. Khan, X.Y. Perrard, G. Brunner, H. Lui, L.M. Sparks, S.R. Smith, X. Wang, Z.Z. Shi, D.E. Lewis, H. Wu, C.M. Ballantyne, Intermuscular and perimuscular fat expansion in obesity correlates with skeletal muscle $\mathrm{T}$ cell and macrophage infiltration and insulin resistance, Int J Obes (Lond) 39(11) (2015) 1607-18.

[51] L.V. Yuzefovych, S.I. Musiyenko, G.L. Wilson, L.I. Rachek, Mitochondrial DNA damage and dysfunction, and oxidative stress are associated with endoplasmic reticulum stress, protein degradation and apoptosis in high fat diet-induced insulin resistance mice, PloS one 8(1) (2013) e54059.

[52] R. Schierwagen, L. Maybuchen, S. Zimmer, K. Hittatiya, C. Back, S. Klein, F.E. Uschner, W. Reul, P. Boor, G. Nickenig, C.P. Strassburg, C. Trautwein, J. Plat, D. Lutjohann, T. Sauerbruch, F. Tacke, J. Trebicka, Seven weeks of Western diet in apolipoprotein-E-deficient mice induce metabolic syndrome and non-alcoholic steatohepatitis with liver fibrosis, Sci Rep 5 (2015) 12931.

[53] S.M. Hofmann, D. Perez-Tilve, T.M. Greer, B.A. Coburn, E. Grant, J.E. Basford, M.H. Tschop, D.Y. Hui, Defective lipid delivery modulates glucose tolerance and metabolic response to diet in apolipoprotein E-deficient mice, Diabetes 57(1) (2008) 5-12.

[54] E.A. Karavia, D.J. Papachristou, I. Kotsikogianni, I. Giopanou, K.E. Kypreos, Deficiency in apolipoprotein $\mathrm{E}$ has a protective effect on diet-induced nonalcoholic fatty liver disease in mice, FEBS J 278(17) (2011) 3119-29.

[55] T. Chiba, M. Ikeda, K. Umegaki, T. Tomita, Estrogen-dependent activation of neutral cholesterol ester hydrolase underlying gender difference of atherogenesis in apoE-/mice, Atherosclerosis 219(2) (2011) 545-51. 
[56] J. Gao, H. Katagiri, Y. Ishigaki, T. Yamada, T. Ogihara, J. Imai, K. Uno, Y. Hasegawa, M. Kanzaki, T.T. Yamamoto, S. Ishibashi, Y. Oka, Involvement of apolipoprotein E in excess fat accumulation and insulin resistance, Diabetes 56(1) (2007) 24-33.

[57] Z.H. Huang, D. Gu, T. Mazzone, Role of adipocyte-derived apoE in modulating adipocyte size, lipid metabolism, and gene expression in vivo, Am J Physiol Endocrinol Metab 296(5) (2009) E1110-9.

[58] D. Cabrera, A. Ruiz, C. Cabello-Verrugio, E. Brandan, L. Estrada, M. Pizarro, N. Solis, J. Torres, F. Barrera, M. Arrese, Diet-Induced Nonalcoholic Fatty Liver Disease Is Associated with Sarcopenia and Decreased Serum Insulin-Like Growth Factor-1, Dig Dis Sci 61(11) (2016) 3190-3198.

[59] H. Eshima, Y. Tamura, S. Kakehi, N. Kurebayashi, T. Murayama, K. Nakamura, R. Kakigi, T. Okada, T. Sakurai, R. Kawamori, H. Watada, Long-term, but not short-term high-fat diet induces fiber composition changes and impaired contractile force in mouse fast-twitch skeletal muscle, Physiol Rep 5(7) (2017).

[60] L.K. Philp, L.K. Heilbronn, A. Janovska, G.A. Wittert, Dietary enrichment with fish oil prevents high fat-induced metabolic dysfunction in skeletal muscle in mice, PloS one 10(2) (2015) e0117494.

[61] K.E. Shortreed, M.P. Krause, J.H. Huang, D. Dhanani, J. Moradi, R.B. Ceddia, T.J. Hawke, Muscle-specific adaptations, impaired oxidative capacity and maintenance of contractile function characterize diet-induced obese mouse skeletal muscle, PloS one 4(10) (2009) e7293.

[62] S.M. Turpin, J.G. Ryall, R. Southgate, I. Darby, A.L. Hevener, M.A. Febbraio, B.E. Kemp, G.S. Lynch, M.J. Watt, Examination of 'lipotoxicity' in skeletal muscle of high-fat fed and ob/ob mice, J Physiol 587(Pt 7) (2009) 1593-605.

[63] S. Timmers, J. de Vogel-van den Bosch, N. de Wit, G. Schaart, D. van Beurden, M. Hesselink, R. van der Meer, P. Schrauwen, Differential effects of saturated versus unsaturated dietary fatty acids on weight gain and myocellular lipid profiles in mice, Nutr Diabetes 1 (2011) e11. 
[64] J. Liu, X. Liang, D. Zhou, L. Lai, L. Xiao, L. Liu, T. Fu, Y. Kong, Q. Zhou, R.B. Vega, M.S. Zhu, D.P. Kelly, X. Gao, Z. Gan, Coupling of mitochondrial function and skeletal muscle fiber type by a miR-499/Fnip1/AMPK circuit, EMBO Mol Med 8(10) (2016) $1212-$ 1228.

[65] A.A. Al-Shammari, E.A. Gaffney, S. Egginton, Modelling capillary oxygen supply capacity in mixed muscles: capillary domains revisited, J Theor Biol 356 (2014) 47-61.

[66] A. Bosutti, S. Egginton, Y. Barnouin, B. Ganse, J. Rittweger, H. Degens, Local capillary supply in muscle is not determined by local oxidative capacity, J Exp Biol 218(Pt 21) (2015) 3377-80.

[67] K. Zibara, E. Malaud, J.L. McGregor, CD36 mRNA and Protein Expression Levels Are Significantly Increased in the Heart and Testis of apoE Deficient Mice in Comparison to Wild Type (C57BL/6), J Biomed Biotechnol 2(1) (2002) 14-21.

[68] M. Akaaboune, M. Villanova, B.W. Festoff, M. Verdiere-Sahuque, D. Hantai, Apolipoprotein E expression at neuromuscular junctions in mouse, rat and human skeletal muscle, FEBS letters 351(2) (1994) 246-8.

[69] S. Fukada, A. Uezumi, M. Ikemoto, S. Masuda, M. Segawa, N. Tanimura, H. Yamamoto, Y. Miyagoe-Suzuki, S. Takeda, Molecular signature of quiescent satellite cells in adult skeletal muscle, Stem cells 25(10) (2007) 2448-59.

[70] R. Rinnankoski-Tuikka, J.J. Hulmi, S. Torvinen, M. Silvennoinen, M. Lehti, R. Kivela, H. Reunanen, U.M. Kujala, H. Kainulainen, Lipid droplet-associated proteins in high-fat fed mice with the effects of voluntary running and diet change, Metabolism 63(8) (2014) 1031-40.

[71] R. Kato, C. Mori, K. Kitazato, S. Arata, T. Obama, M. Mori, K. Takahashi, T. Aiuchi, T. Takano, H. Itabe, Transient increase in plasma oxidized LDL during the progression of atherosclerosis in apolipoprotein E knockout mice, Arterioscler Thromb Vasc Biol 29(1) (2009) 33-9.

[72] C. Bonnard, A. Durand, S. Peyrol, E. Chanseaume, M.A. Chauvin, B. Morio, H. Vidal, J. Rieusset, Mitochondrial dysfunction results from oxidative stress in the skeletal muscle of 
diet-induced insulin-resistant mice, The Journal of clinical investigation 118(2) (2008) 789-800.

[73] J. Ciapaite, S.A. van den Berg, S.M. Houten, K. Nicolay, K.W. van Dijk, J.A. Jeneson, Fiber-type-specific sensitivities and phenotypic adaptations to dietary fat overload differentially impact fast- versus slow-twitch muscle contractile function in C57BL/6J mice, The Journal of nutritional biochemistry 26(2) (2015) 155-64.

[74] C.R. Hancock, D.H. Han, M. Chen, S. Terada, T. Yasuda, D.C. Wright, J.O. Holloszy, High-fat diets cause insulin resistance despite an increase in muscle mitochondria, Proc Natl Acad Sci U S A 105(22) (2008) 7815-20.

[75] J. Hoeks, J. Wilde, M.F. Hulshof, S.A. Berg, G. Schaart, K.W. Dijk, E. Smit, E.C. Mariman, High fat diet-induced changes in mouse muscle mitochondrial phospholipids do not impair mitochondrial respiration despite insulin resistance, PloS one 6(11) (2011) e27274.

[76] J.F. Young, L.B. Larsen, A. Malmendal, N.C. Nielsen, I.K. Straadt, N. Oksbjerg, H.C. Bertram, Creatine-induced activation of antioxidative defence in myotube cultures revealed by explorative NMR-based metabonomics and proteomics, Journal of the International Society of Sports Nutrition 7(1) (2010) 9.

[77] U. Warskulat, E. Borsch, R. Reinehr, B. Heller-Stilb, C. Roth, M. Witt, D. Haussinger, Taurine deficiency and apoptosis: findings from the taurine transporter knockout mouse, Archives of biochemistry and biophysics 462(2) (2007) 202-9.

[78] N. Ferre, M. Martinez-Clemente, M. Lopez-Parra, A. Gonzalez-Periz, R. Horrillo, A. Planaguma, J. Camps, J. Joven, A. Tres, F. Guardiola, R. Bataller, V. Arroyo, J. Claria, Increased susceptibility to exacerbated liver injury in hypercholesterolemic ApoE-deficient mice: potential involvement of oxysterols, Am J Physiol Gastrointest Liver Physiol 296(3) (2009) G553-62.

[79] M. Martinez-Clemente, N. Ferre, E. Titos, R. Horrillo, A. Gonzalez-Periz, E. MoranSalvador, C. Lopez-Vicario, R. Miquel, V. Arroyo, C.D. Funk, J. Claria, Disruption of the 
12/15-lipoxygenase gene (Alox15) protects hyperlipidemic mice from nonalcoholic fatty liver disease, Hepatology 52(6) (2010) 1980-91.

[80] S. Boudina, S. Sena, C. Sloan, A. Tebbi, Y.H. Han, B.T. O'Neill, R.C. Cooksey, D. Jones, W.L. Holland, D.A. McClain, E.D. Abel, Early mitochondrial adaptations in skeletal muscle to diet-induced obesity are strain dependent and determine oxidative stress and energy expenditure but not insulin sensitivity, Endocrinology 153(6) (2012) 2677-88.

[81] M. Raman, W. Chen, M.H. Cobb, Differential regulation and properties of MAPKs, Oncogene 26(22) (2007) 3100-12.

[82] S.K. Powers, J. Duarte, A.N. Kavazis, E.E. Talbert, Reactive oxygen species are signalling molecules for skeletal muscle adaptation, Experimental physiology 95(1) (2010) $1-9$.

[83] A.S. Anderson, K.R. Haynie, R.P. McMillan, K.L. Osterberg, N.E. Boutagy, M.I. Frisard, B.M. Davy, K.P. Davy, M.W. Hulver, Early skeletal muscle adaptations to short-term highfat diet in humans before changes in insulin sensitivity, Obesity 23(4) (2015) 720-4.

[84] A. Sadeghi, S.S. Seyyed Ebrahimi, A. Golestani, R. Meshkani, Resveratrol Ameliorates Palmitate-Induced Inflammation in Skeletal Muscle Cells by Attenuating Oxidative Stress and JNK/NF-kappaB Pathway in a SIRT1-Independent Mechanism, Journal of cellular biochemistry 118(9) (2017) 2654-2663.

[85] B. Seaberg, G. Henslee, S. Wang, X. Paez-Colasante, G.E. Landreth, M. Rimer, Muscle-derived extracellular signal-regulated kinases 1 and 2 are required for the maintenance of adult myofibers and their neuromuscular junctions, Molecular and cellular biology 35(7) (2015) 1238-53.

[86] R. Stewart, L. Flechner, M. Montminy, R. Berdeaux, CREB is activated by muscle injury and promotes muscle regeneration, PloS one 6(9) (2011) e24714.

[87] I. Garcia-Ruiz, P. Solis-Munoz, D. Fernandez-Moreira, M. Grau, T. Munoz-Yague, J.A. Solis-Herruzo, NADPH oxidase is implicated in the pathogenesis of oxidative phosphorylation dysfunction in mice fed a high-fat diet, Sci Rep 6 (2016) 23664. 
[88] L.C. Joseph, E. Barca, P. Subramanyam, M. Komrowski, U. Pajvani, H.M. Colecraft, M. Hirano, J.P. Morrow, Inhibition of NAPDH Oxidase 2 (NOX2) Prevents Oxidative Stress and Mitochondrial Abnormalities Caused by Saturated Fat in Cardiomyocytes, PloS one 11(1) (2016) e0145750. 
Figures
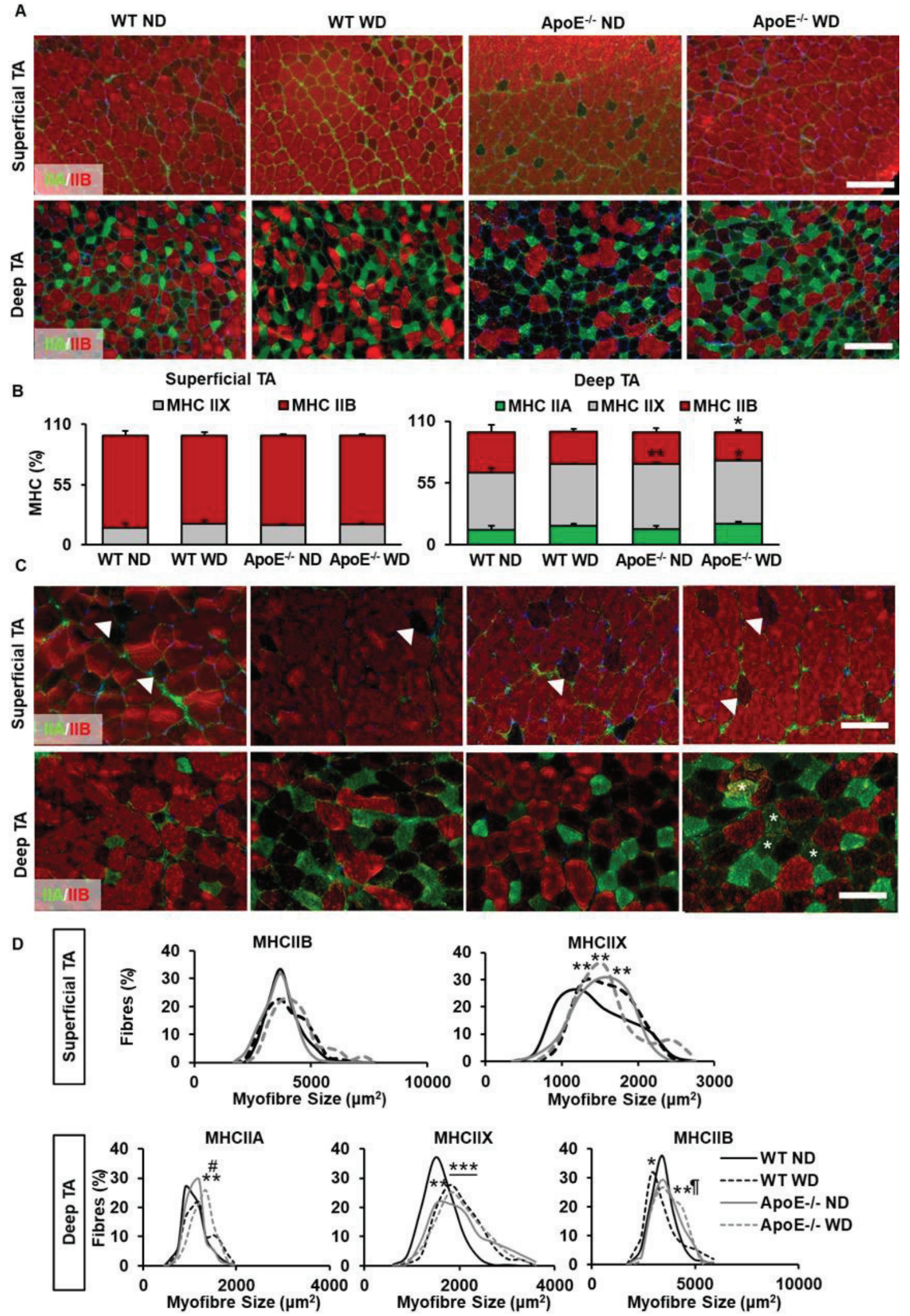
Figure 1. ApoE deficiency and Western-type diet induce a mild transition to oxidative myofibres. Myofibre composition and myofibre size in tibialis anterior was assessed in C57BI/6J (wild-type, WT) and $\mathrm{ApoE}^{-/-}$mice fed a standard chow diet (normal diet, ND) or Western-type diet (WD) for 12 weeks. (A) Representative images of MHC immunostaining in superficial and deep TA (x10 magnification, scale bar $200 \mu \mathrm{m}$ ). (B) Quantification of myofibre type in superficial and deep tibialis anterior. Data are expressed as mean \pm SD. (C) Representative images of $\mathrm{MHC}$ immunostaining in superficial and deep tibialis anterior (x20 magnification, scale bar $100 \mu \mathrm{m}$ ). Arrow heads show type IIX myofibres and asterisks show hybrid IIX/IIA myofibres. (D) Quantification of myofibre size in superficial and deep TA. Data are expressed as frequency distribution. Statistical analysis was performed by two-way ANOVA for myofibre type (Main effects: Genotype: $p<0.01$ for MHCIIB and MHCIIX; Diet $p<0.01$ for MHCIIB, and MHCIIA; Interaction; $p=0.042$ for MHCIIX in deep TA) followed by the Bonferroni post hoc tests for myofibre type and by $x^{2}$ square for myofibre size distribution, ${ }^{*} p<0.05,{ }^{* *} p<0.01,{ }^{* * *} p<0.001$ vs WT ND, $\#<0.05$ vs $A p o E^{-/-}$ND and $\uparrow<0.05$ vs WT WD (n=6/group). In superficial TA: WT WD versus WT ND: $X^{2}=8.105 ; \mathrm{df}=9 ; \mathrm{p}=0.524$ for type IIB and $X^{2}=17.217 ; \mathrm{df}=6 ; p<0.01$ for type IIX. For $A p o E^{-/-}$ND versus WT ND; $X^{2}=8.078 ; \mathrm{df}=9 ; p=0.526$ for type IIB and $X^{2}=17.957 ; \mathrm{df}=6 ; p<0.01$ for type IIX. ApoE ${ }^{-/-}$WD versus WT ND: $X^{2}=17.116 ; \mathrm{df}=11 ; \mathrm{p}=0.104$ for type IIB and $X^{2}=21.201 ; \mathrm{df}=7 ; \mathrm{p}<0.01$ for type IIX. Moreover, ApoE ${ }^{--}$WD versus $A p o E^{-/-} \mathrm{ND} ; X^{2}=30.787 ; \mathrm{df}=12 ; \mathrm{p}=0.064$ for type IIB and $X^{2}=19.863 ; \mathrm{df}=7 ; p<0.01$ for type IIX. In deep TA: WT WD versus WT ND; $X^{2}=15.372 ; \mathrm{df}=8 ; p<0.05$ for type IIB, $X^{2}=41.191 ; \mathrm{df}=9 ; p<0.001$ for type IIX and $x^{2}=11.065 ; \mathrm{df}=9 ; \mathrm{p}=0.064$ for type IIA. ApoE ${ }^{-/-}$ND versus WT ND; $x^{2}=8.568 ; \mathrm{df}=8 ; p=0.38$ for type IIB, $X^{2}=36.292$, df=10, $p<0.01$ for type IIX and $X^{2}=4.507 ; \mathrm{df}=8 ; p=0.809$ for type IIA. $\mathrm{ApoE}^{-/-}$WD versus WT ND; $X^{2}=14.962 ; \mathrm{df}=7 ; p<0.01$ for type IIB, $X^{2}=42.547 ; \mathrm{df}=9 ; p<0.001$ for type IIX and $X^{2}=29.592 ; \mathrm{df}=9 ; p<0.001$ for type IIA. ApoE ${ }^{-/-}$WD versus $\mathrm{ApoE}^{-/-} \mathrm{ND}$ : $X^{2}=3.696 ; \mathrm{df}=8 ; p=0.883$ for type IIB, $X^{2}=4.844 ; \mathrm{df}=9, \quad \mathrm{p}=0.848$ for type IIX and $x^{2}=27.910 ; \mathrm{df}=9 ; \mathrm{p}<0.05$ for type IIA. 
A
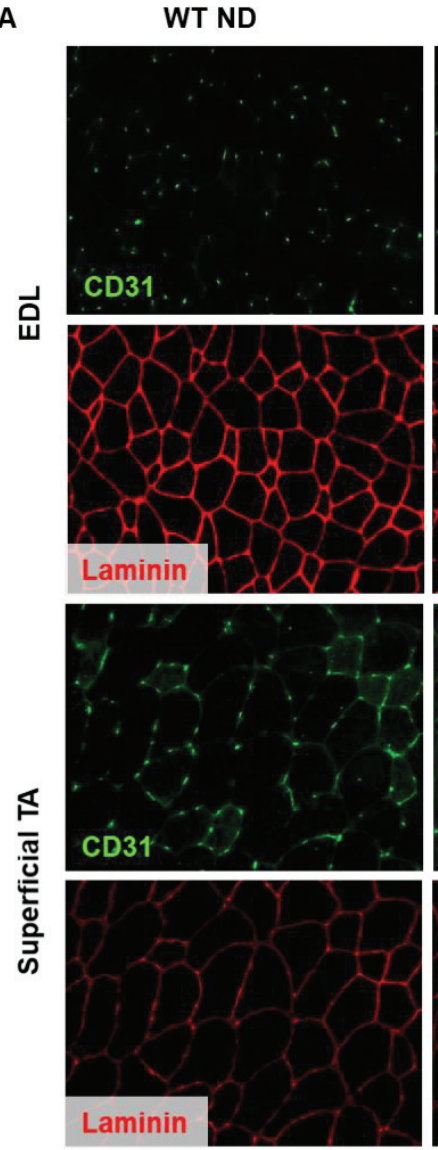

B

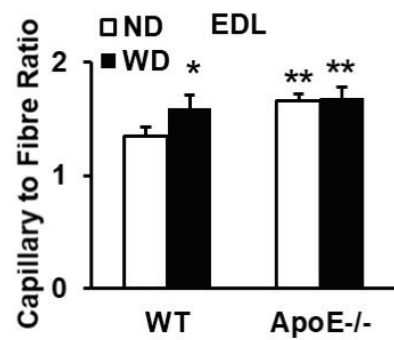

WT WD
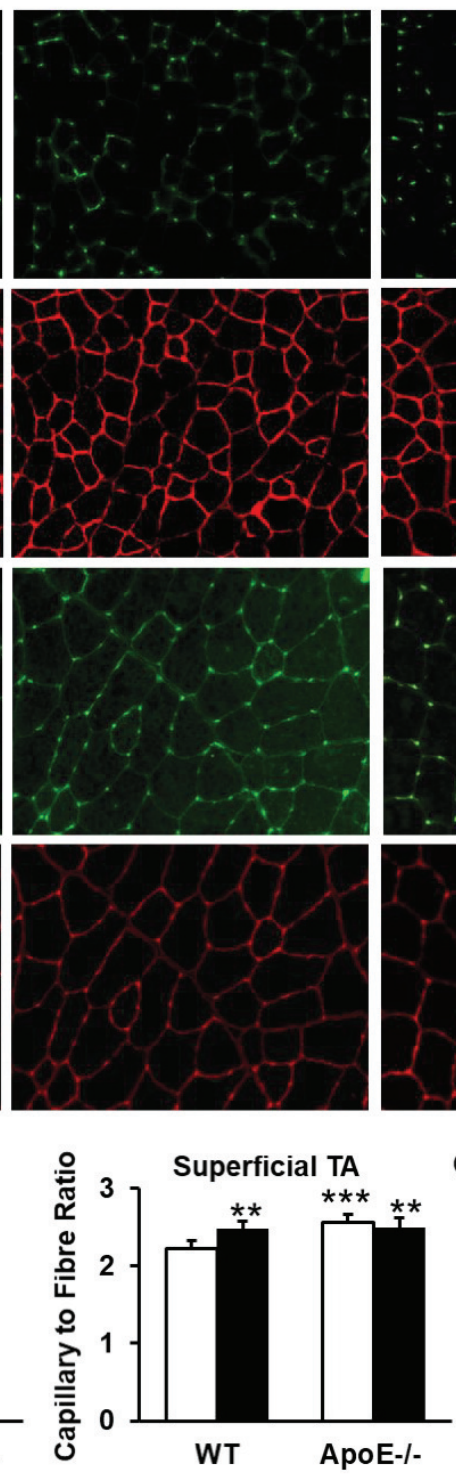

ApoE $^{-/-}$ND
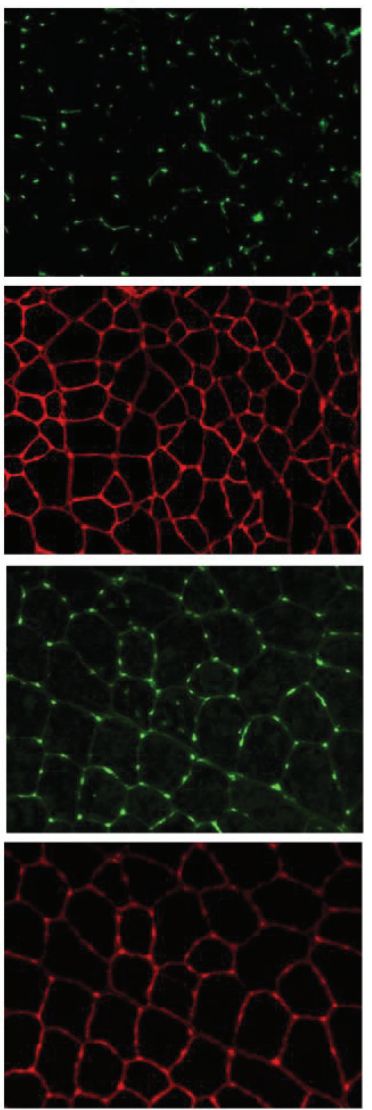

C

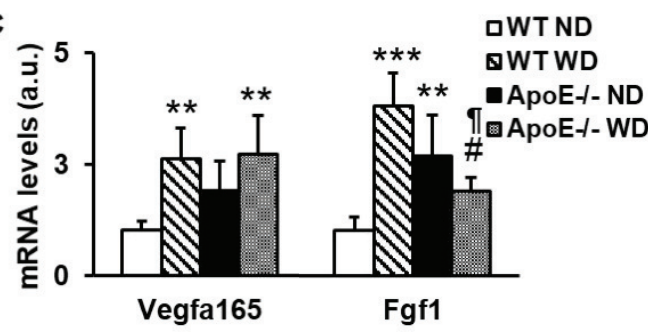

Figure 2. ApoE deficiency and Western-type diet induce an increase in capillary density and angiogenic gene expression. Capillary density of skeletal muscle was assessed in C57BI/6J (wild-type, WT) and $\mathrm{ApoE}^{-/}$mice fed a standard chow diet (normal diet, ND) or Western-type diet (WD) for 12 weeks. (A) Representative images of CD31 immunostaining - a marker of endothelial cells - and laminin - a protein of the extracellular matrix - in EDL and superficial TA (x20 magnification, scale bar $100 \mu \mathrm{m}$ ). (B) Quantification of capillary density in EDL and superficial TA muscles. Data are expressed as mean \pm SD. (C) mRNA levels of key angiogenic factors in the gastrocnemius. Data are expressed as mean \pm SD and shown as fold increase relative to WT ND in arbitrary units. Statistical analysis was performed by two-way ANOVA (Main effects: Genotype: $p<0.05$ for CD31 in both muscles; Diet $p<0.05$ for CD31 in both muscles and Vegfa165 and Fgf1; Interaction; $\mathrm{p}<0.01$ for CD31 in both muscle beds and Fgf1 gene) followed by Bonferroni post hoc tests, ${ }^{*} p<0.05,{ }^{* *} p<0.01,{ }^{* * *} p<0.001$ vs WT ND, \#<0.05 vs $A p o E^{-/-}$ND and $\mathbb{T}<0.05$ vs WT WD ( $n=6 /$ group). 
A

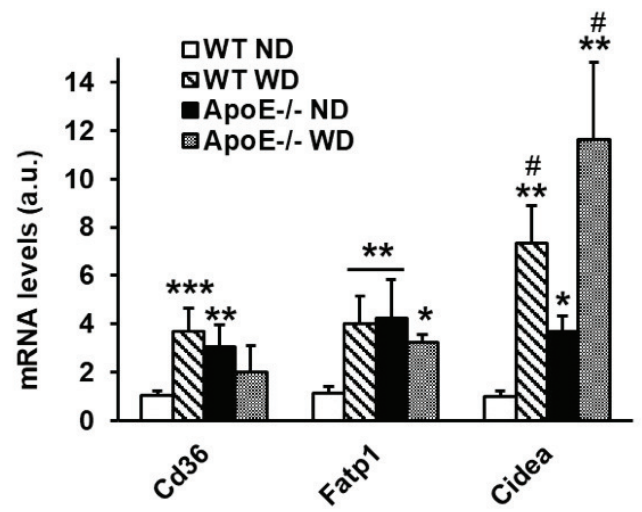

C
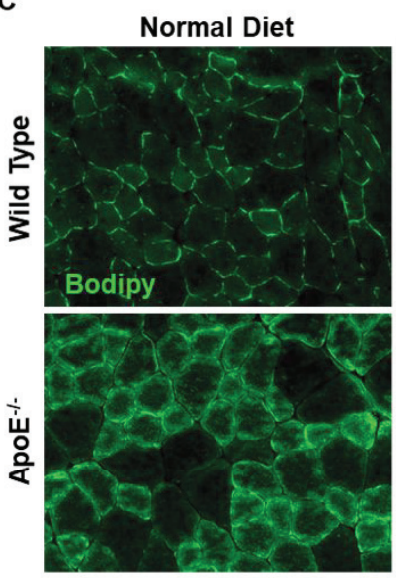

Tibialis anterior

D

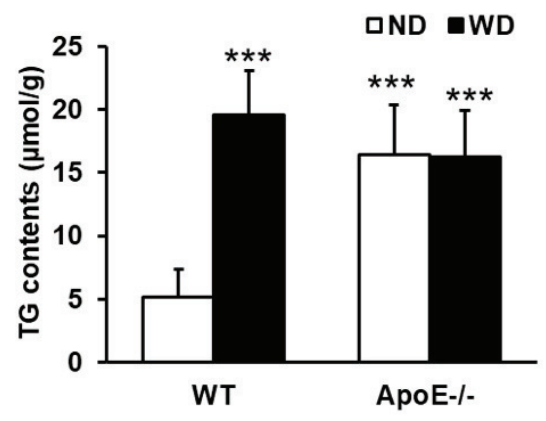

B
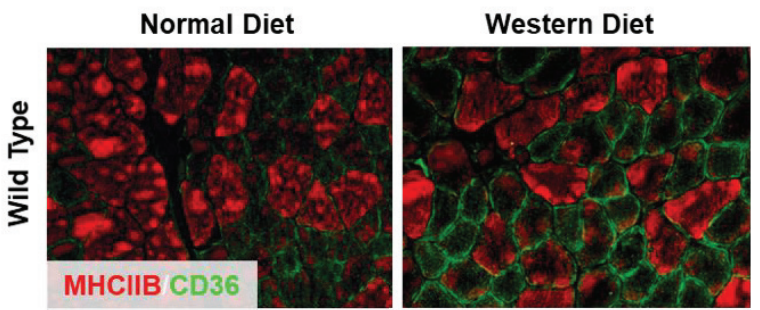

这
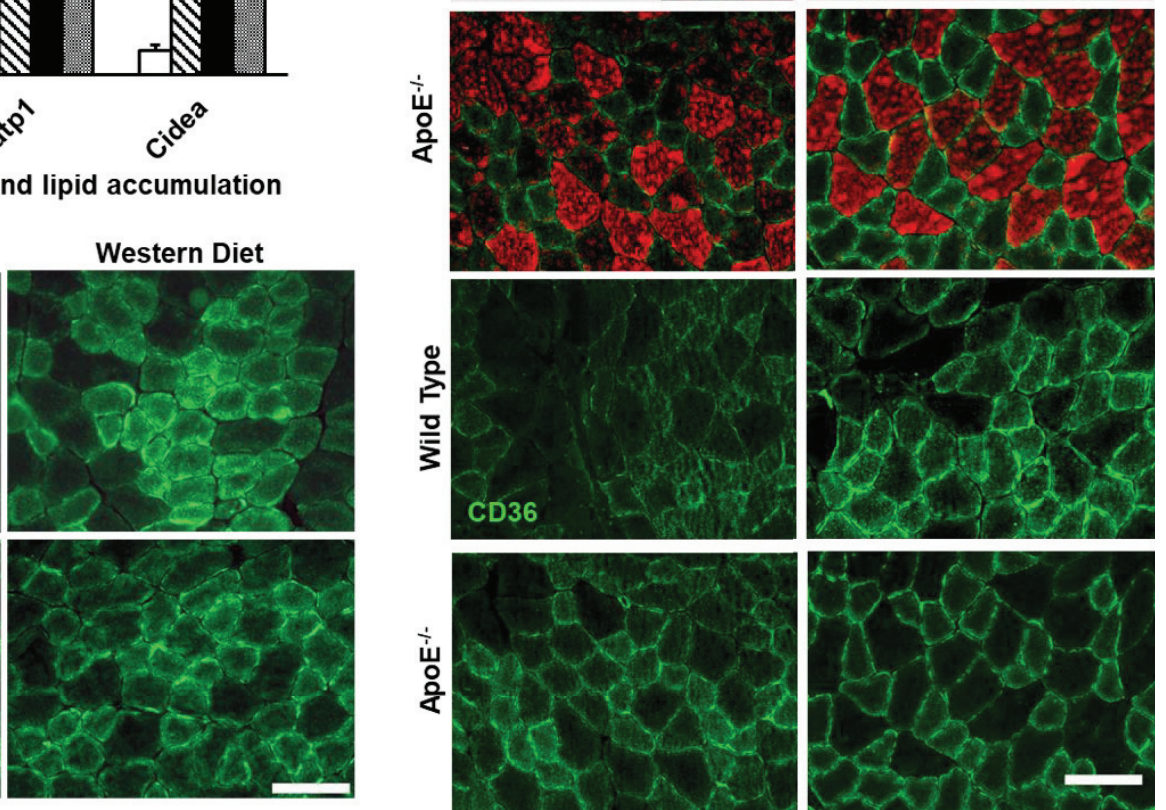

CD36

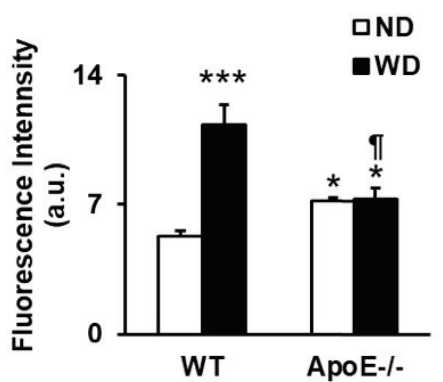

Figure 3. ApoE deficiency and Western-type diet increase independently intramyocellular fat content. Ectopic fat accumulation in skeletal muscle was assessed in C57BI/6J (wild-type, WT) and $\mathrm{ApoE}^{-/-}$mice fed a standard chow diet (normal diet, ND) or Western-type diet (WD) for 12 weeks. (A) mRNA levels of genes involved in fatty acid transport and lipid droplet regulation. Data are expressed as mean \pm SD and shown as fold increase relative to WT ND in arbitrary units. (B) Representative images of MHCIIB and CD36 in the TA (x20 magnification, scale bar $100 \mu \mathrm{m})$ and quantification of CD36 fluorescence intensity. Data are expressed as mean \pm SD. (C) Representative images of Bodipy staining for lipids in the Tibialis Anterior (x20 magnification, $100 \mu \mathrm{m}$ for Bodipy staining). (D) Triacylglycerol (TG) content in tibialis anterior as determined by gas chromatography. Data are expressed as mean \pm SD. Statistical analysis was performed by two-way ANOVA (Main effects: Genotype: $p<0.05$ for Fatp1 and Cidea, triacylglycerol content and CD36 fluorescence intensity; Diet $\mathrm{p} \leq 0.01$ for $\mathrm{Cd} 36$ and Cidea, triacylglycerol content and CD36 fluorescence intensity; Interaction; $p \leq 0.01$ for Cd36 and Fatp1 and 
triacylglycerol content) followed by Bonferroni post hoc tests, ${ }^{*} p<0.05,{ }^{* *} p<0.01,{ }^{* * *} p<0.001$ vs WT ND, \#<0.05 vs $A p o E^{-/-}$ND and $\mathbb{\Uparrow}<0.05$ vs WT WD (n=6/group). 
A

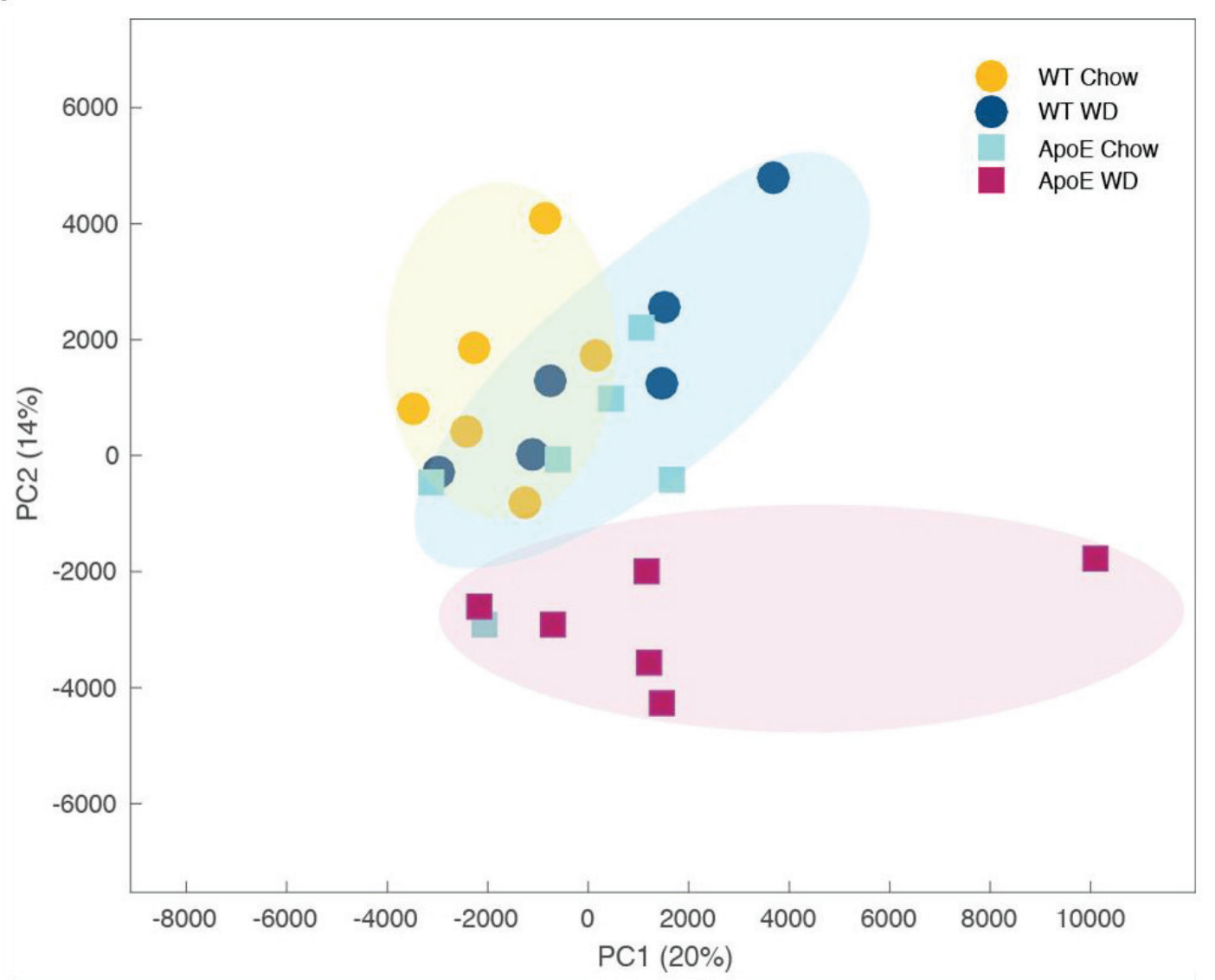

B

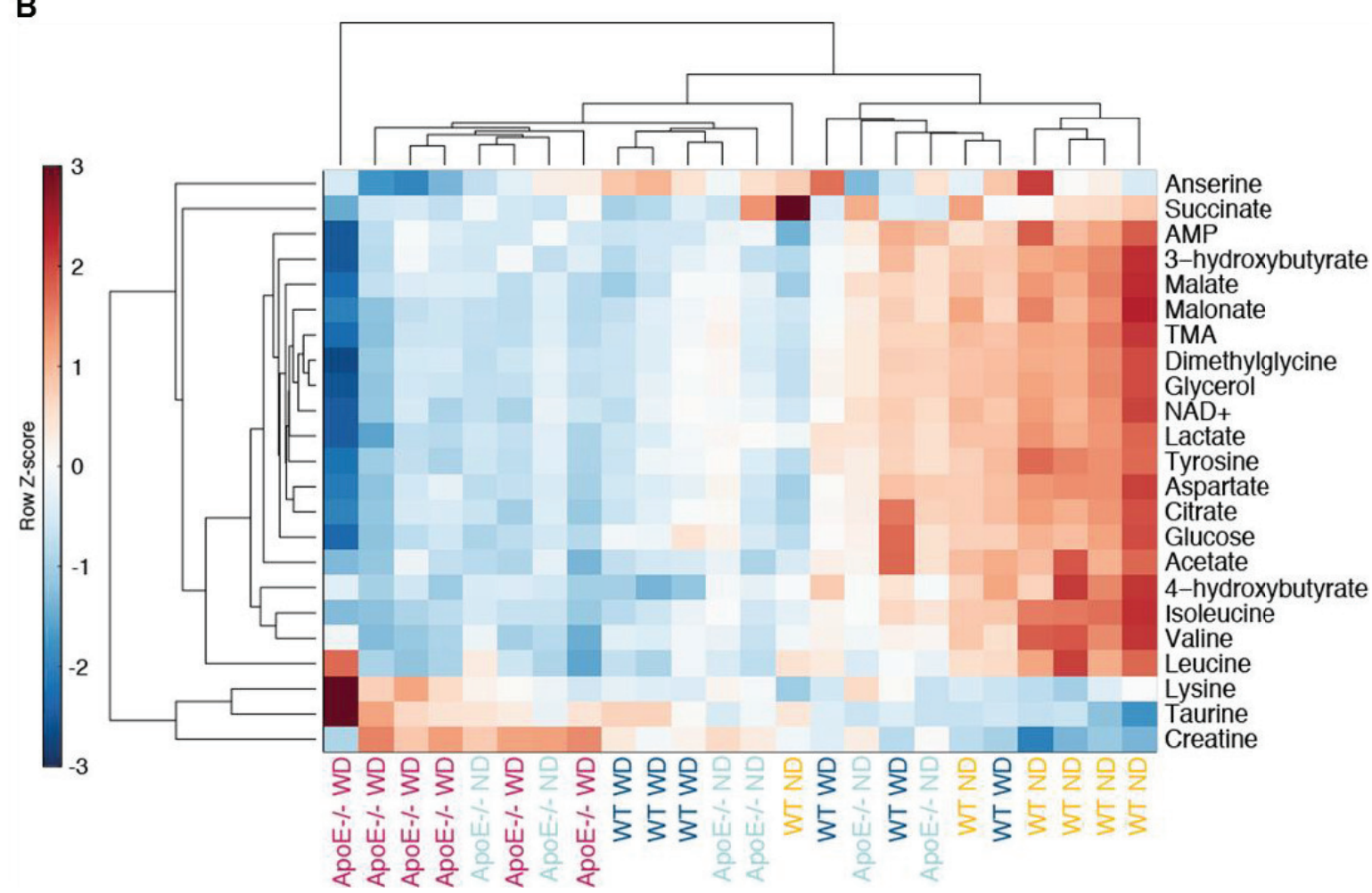

Figure 4. ApoE deficiency and Western-type diet independently induce changes in metabolic phenotype of skeletal muscle. (A) PCA scores plot of the metabolic profiles 
obtained from skeletal muscle of C57BI/6J (wild-type, WT) and $\mathrm{ApoE}^{-/-}$mice fed a standard chow diet (normal diet, ND) or Western-type diet (WD) for 12 weeks. (B) Unsupervised hierarchical clustering heat-map of the metabolites from the skeletal muscles of all mice. Each row represents a metabolite and each column represents a sample. The row Z-score of each metabolite is plotted, representing the intensity of each metabolite across samples ( $n=6 /$ group). 


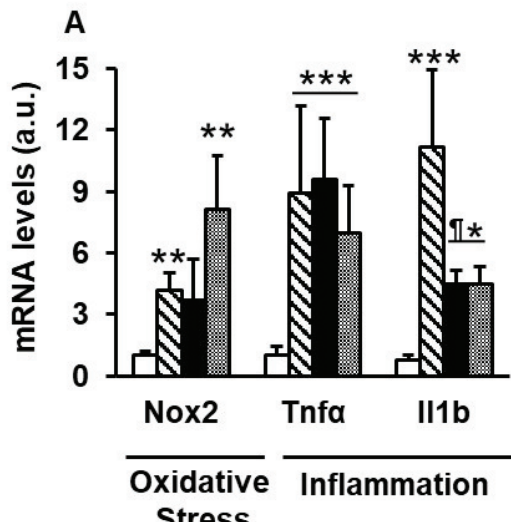
Stress
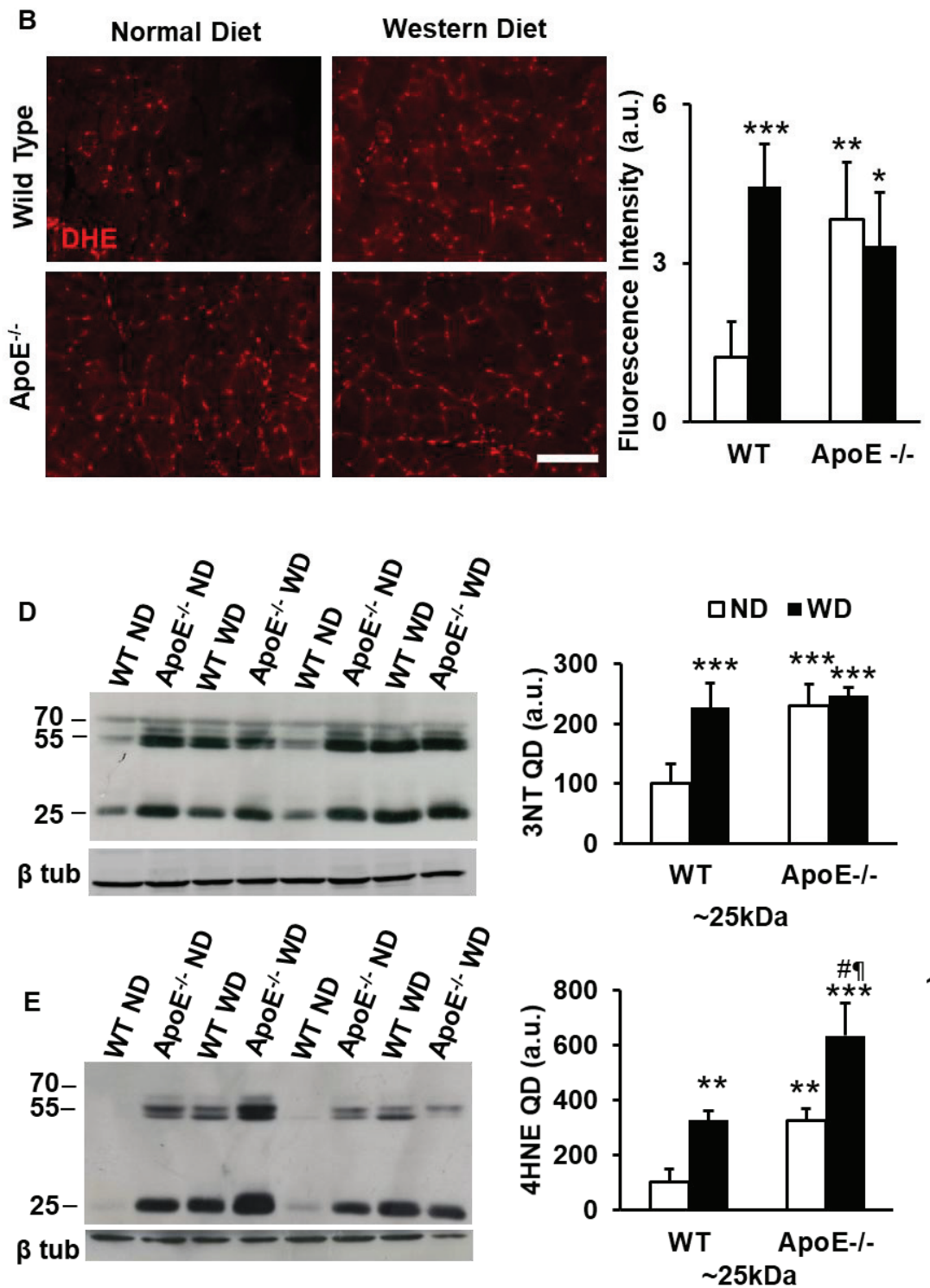

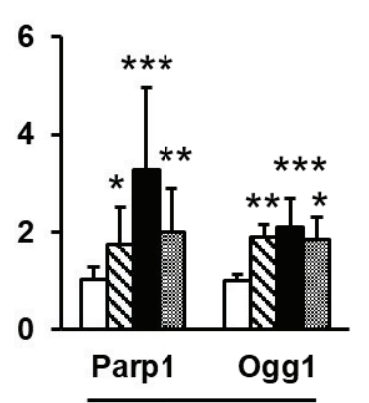

DNA Repair Enzymes
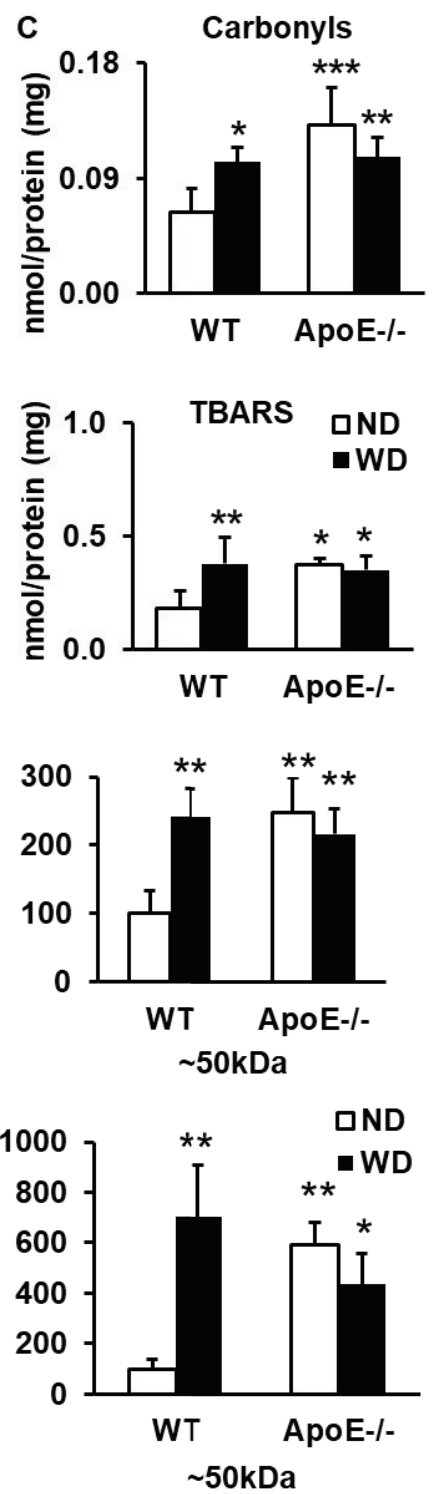

Figure 5. ApoE deficiency and Western-type diet independently increase oxidative stress in skeletal muscle. Oxidative stress in skeletal muscle was assessed in C57BI/6J (wild-type, WT) and $\mathrm{ApoE}^{-/-}$mice fed a standard chow diet (normal diet, ND) or Western-type diet (WD) for 12 weeks. (A) mRNA levels in the gastrocnemius of a gene involved in oxidative stress (Nox2), inflammatory genes (Tnfa, //1b), antioxidant genes (Gpx1, Gpx4, 
Prdx1, Sod1, Mgst1 and Cat) and genes involved in DNA repair (Parp1, Ogg1). Data are expressed as mean \pm SD and shown as fold increase relative to WT ND in arbitrary units. (B) Representative images for free radical staining with $10 \mu \mathrm{M}$ DHE and quantification of DHE fluorescence intensity (x20 magnification, scale bar $100 \mu \mathrm{m}$ ). (C) Protein carbonyl contents and TBARS assay in protein lysates of quadriceps. Data are expressed as mean \pm SD after normalisation to protein content. (D) Immunoblot in protein lysates of quadriceps (QD) for tyrosine nitration (3NT). Data are expressed as mean \pm SD and data are shown as fold increase relative to WT ND after normalisation to $\beta$ tubulin. (E) Immunoblot in protein lysates of quadriceps for $4 \mathrm{HNE}$ adducts in histidine residues. Data are expressed as mean \pm SD and shown as fold increase relative to WT ND after normalisation to $\beta$ tubulin). Statistical analysis was performed by two-way ANOVA (Main effects: Genotype: $p<0.05$ for DHE fluorescence, for all protein oxidative modifications and all genes except for Sod1 and Gpx1; Diet $p<0.05$ for DHE fluorescence, TBARS, 3NT and 4HNE protein oxidative modifications and all genes except for Tnfa and Ogg1; Interaction; $\mathrm{p}<0.05$ for TBARS, carbonyls, 3NT and for all genes, except for Tnfa, Cat, Parp1 and Ogg1) followed by Bonferroni post hoc tests, ${ }^{*} \mathrm{p}<0.05,{ }^{* *} \mathrm{p}<0.01,{ }^{* *} \mathrm{p}<0.001$ vs WT ND (n=6/group). 


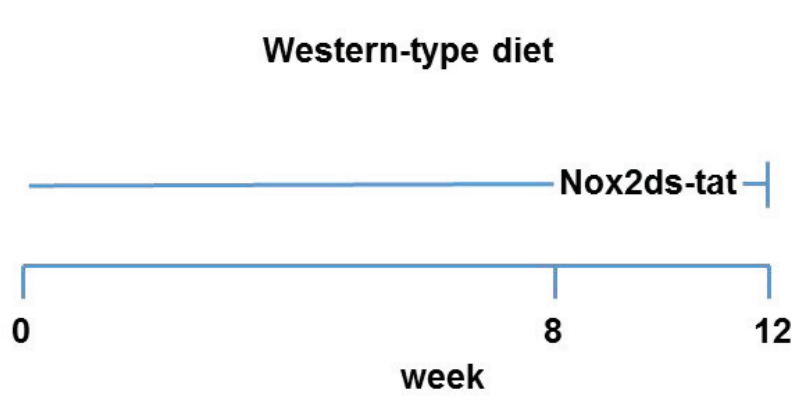

B
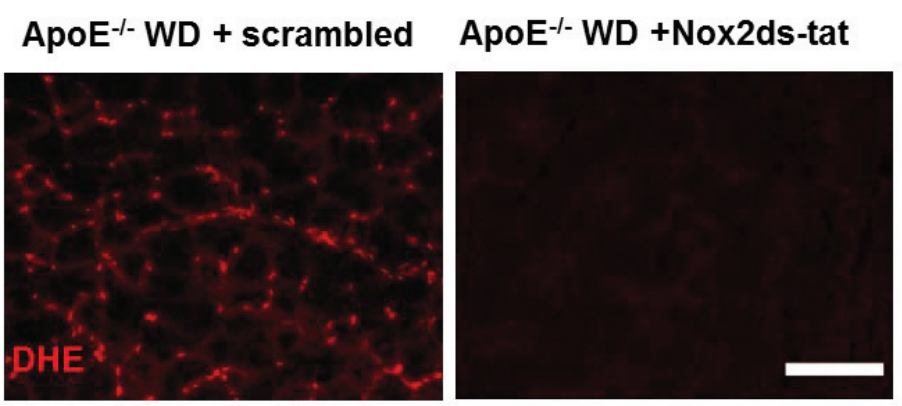
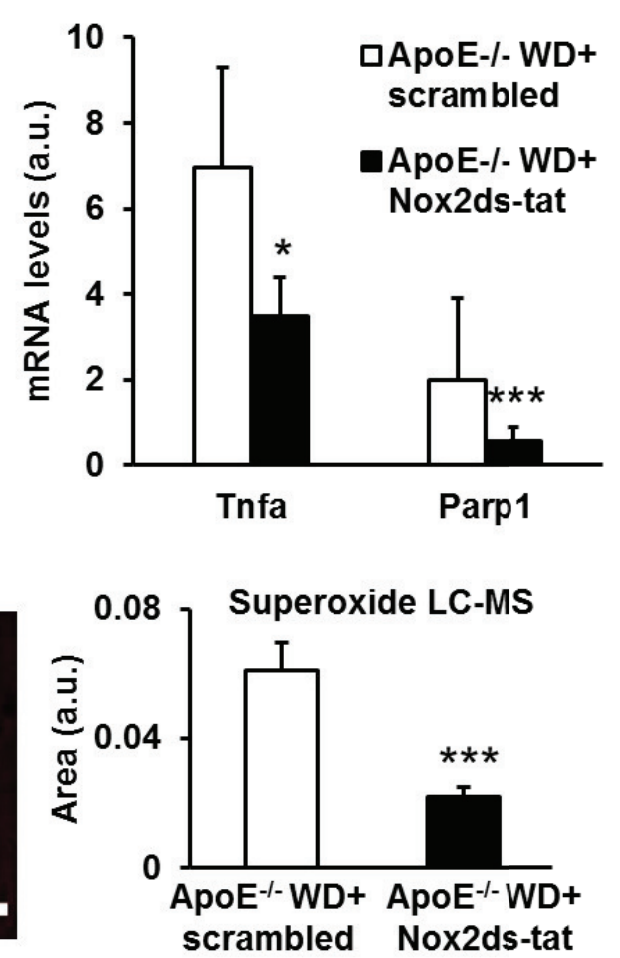

D Protein Carbonylation QD
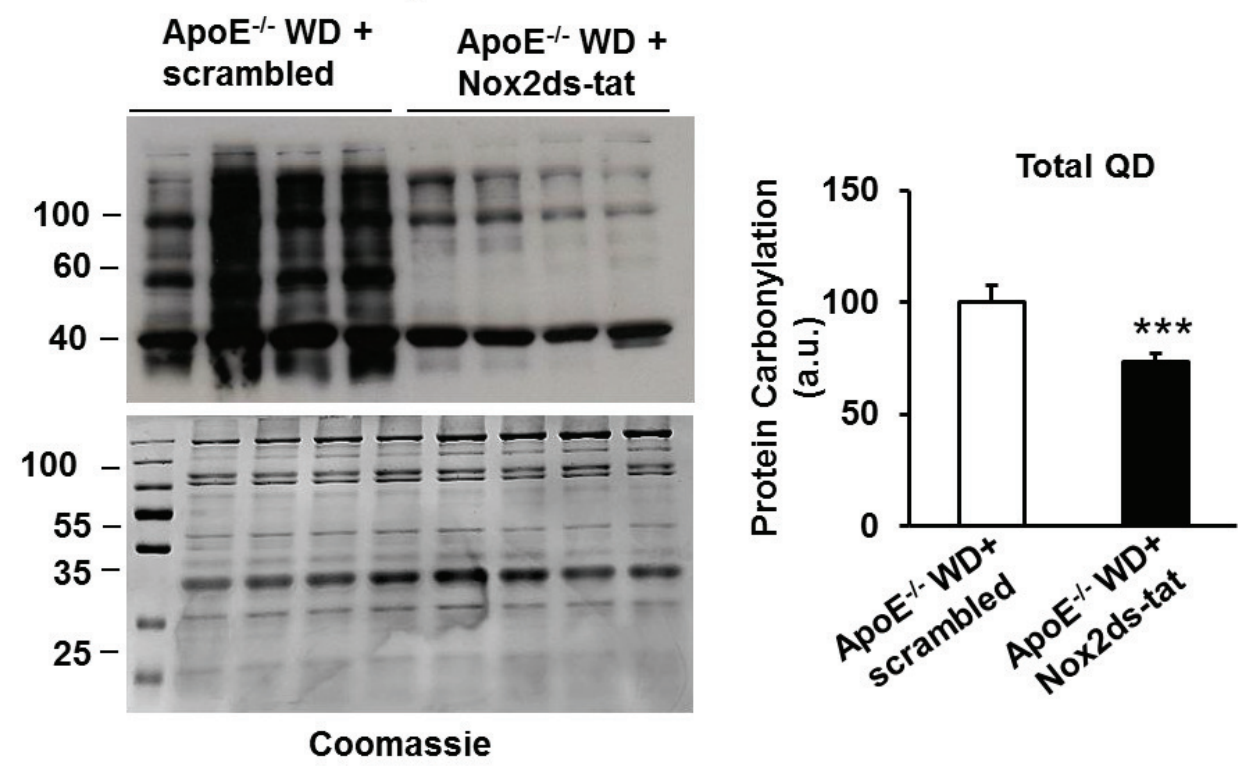

Figure 6. Nox2ds-tat peptide decreases oxidative stress. (A) Oxidative stress was assessed in skeletal muscle of $\mathrm{ApoE}^{-{ }^{--}}$mice fed Western-type diet (WD) for 12 weeks and treated with either Nox2ds-tat peptide (ApoE ${ }^{-/-}$WD + Nox2ds-tat) or scrambled peptide $\left(\mathrm{ApoE}^{-/-} \mathrm{WD}+\mathrm{scrambled}\right)$ for the last 4 weeks of feeding. (B) Representative images for free radical staining was assessed with $10 \mu \mathrm{M}$ DHE in the EDL (x20 magnification, scale bar 100 $\mu \mathrm{m})$ and superoxide production as measured by LC-MS. Data are expressed as mean \pm SD in arbitrary units after normalisation to protein content. (C) mRNA levels of genes involved in 
inflammation and DNA repair of modified bases. Data are expressed as mean \pm SD and shown as relative fold increase ApoE $^{-/}$WD treated with scrambled sequence in arbitrary units. (D) Immunoblot in protein lysates of quadriceps for protein carbonylation. Data are expressed as mean $\pm S D$ and data are shown as fold change relative to $A p o E^{-/-} W D$ treated with scrambled sequence after normalisation to total protein content. Statistical analysis was

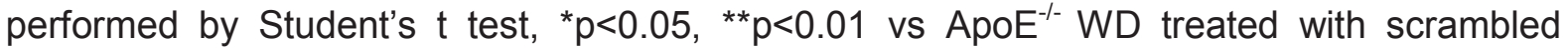
sequence ( $n=6 /$ group). 

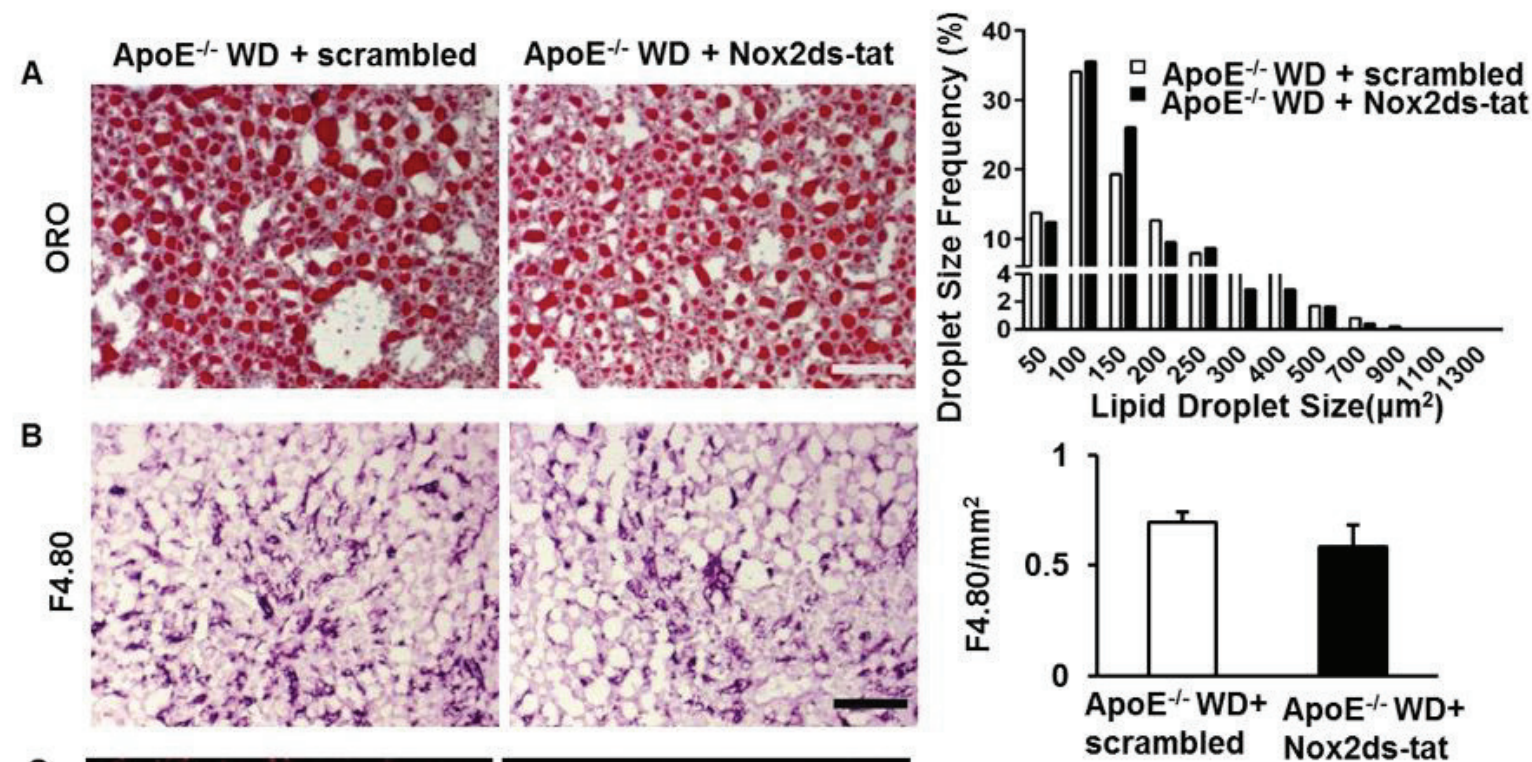

C
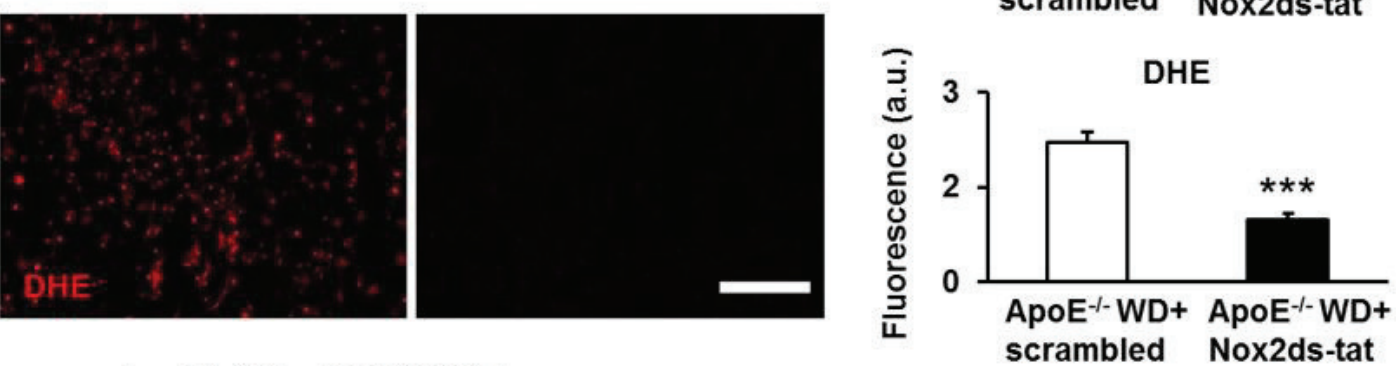

D
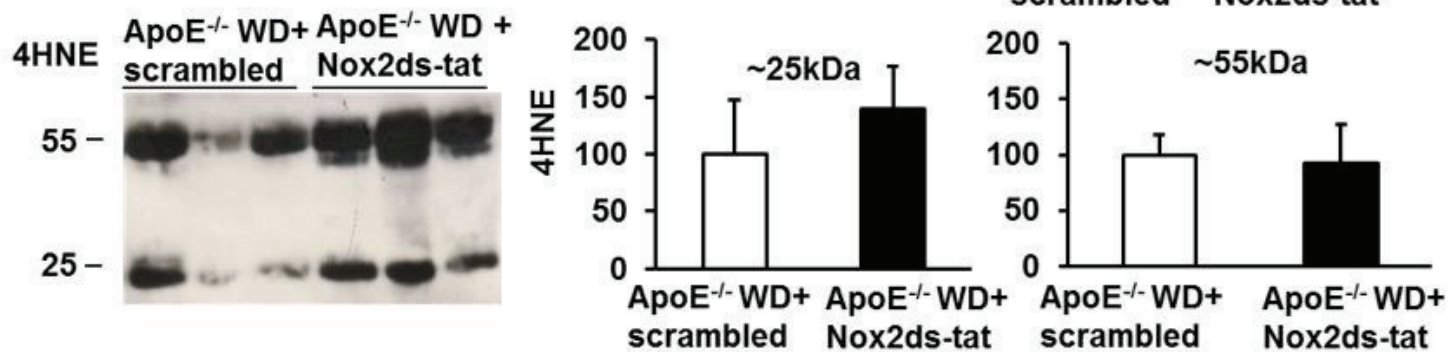

E $3 \mathrm{NT} \quad \mathrm{ApoE}^{-/-} \mathrm{WD}+\mathrm{ApoE}^{-/-} \mathrm{WD}+$

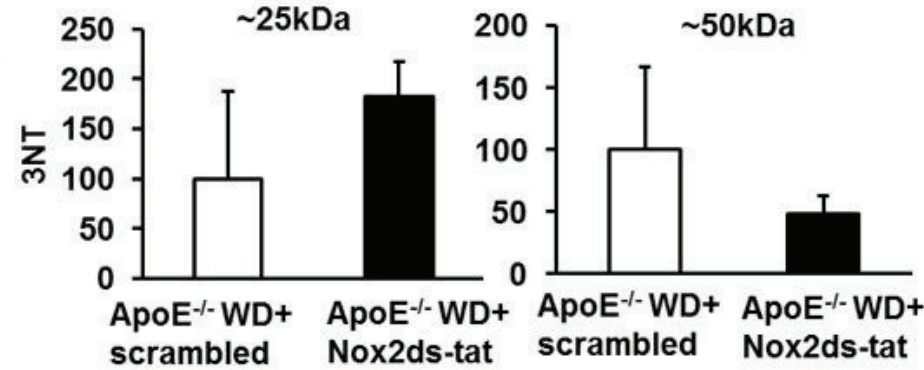

F
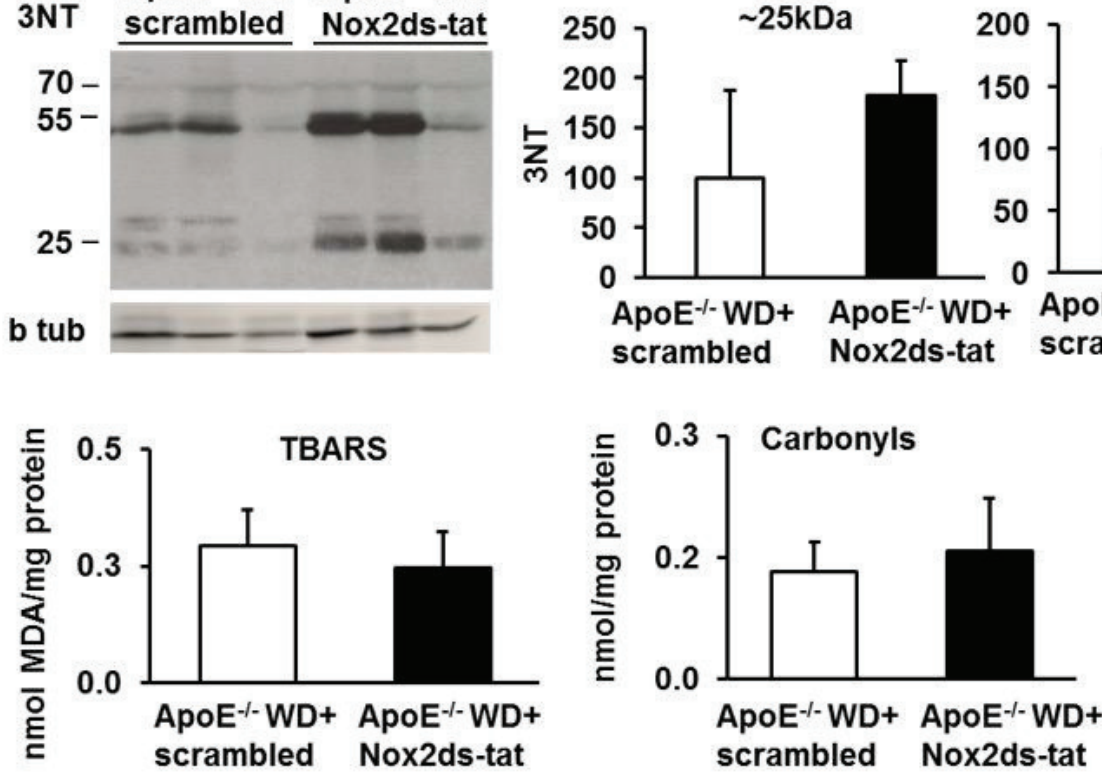
Figure 7. Nox2ds-tat peptide does not alleviate hepatic steatosis and inflammation and decreases ROS. Hepatic fat accumulation, inflammation and oxidative stress was assessed in $\mathrm{ApoE}^{-/-}$mice fed Western-type diet for 12 weeks and treated with either Nox2dstat peptide (ApoE ${ }^{-/-}$WD + Nox2ds-tat) or scrambled peptide (ApoE ${ }^{-/}$WD + scrambled) for the last 4 weeks of feeding. (A) Representative images of Oil Red O staining of lipids in the liver and quantification of lipid droplet size and frequency (x20 magnification, scale bar $100 \mu \mathrm{m}$ ). Data are expressed as percentage of lipid droplet size distribution. (B) Representative images of F4.80 staining in the liver and relative quantification of macrophages per area ( $\mathrm{x} 20$ magnification, scale bar $100 \mu \mathrm{m}$ ). Data are expressed as mean \pm SD. (C) Representative images for free radical staining, assessed with $10 \mu \mathrm{M}$ DHE in the liver (x20 magnification, scale bar $100 \mu \mathrm{m}$ ) and quantification of fluorescence intensity in arbitrary units. Data are expressed as mean \pm SD. (D) Immunoblot in liver protein lysates for $4 \mathrm{HNE}$ adducts in histidine residues and quantification of density. Data are expressed as mean $\pm S D$ and shown as fold increase relative to $A p o E^{-/-} W D$ group treated with scrambled sequence after normalisation to $\beta$ tubulin in arbitrary units. (E) Immunoblot in liver protein lysates for tyrosine nitration (3NT) and quantification of density. Data are expressed as mean \pm SD and shown as fold increase relative to $A p o E^{-/-} W D$ group treated with scrambled sequence after normalisation to $\beta$ tubulin in arbitrary units. (F) TBARS assay and protein carbonyl content in liver. Data are expressed as mean \pm SD. Statistical analysis was performed by Student's $t$ test for inflammation and oxidative stress assays with $p<0.05$ and by $x^{2}$ square for lipid droplet size distribution with $X^{2}=11.34 ; \mathrm{df}=5$ and $p=0.93 \mathrm{vs} \mathrm{ApoE}^{-/-}$WD treated with scrambled sequence ( $n=6 /$ group). 


\section{Online supplementary file}

\section{Detailed Methods}

\section{Histology}

For succinate dehydrogenase $(\mathrm{SDH})$ staining, transverse muscle sections of extensor digitorum longus (EDL), tibialis anterior (TA) and soleus were incubated in a sodium phosphate buffer containing $75 \mathrm{mM}$ sodium succinate (Sigma-Aldrich, UK) and $1.1 \mathrm{mM}$ nitroblue tetrazolium (Sigma-Aldrich, UK). Samples were then fixed in 4\% paraformaldehyde (Sigma-Aldrich) and mounted with hydromount mounting medium (Fisher Scientific, UK).

For Masson's trichrome staining, sections were fixed with 4\% PFA and subsequently incubated with $1 \%(\mathrm{v} / \mathrm{v})$ acetic acid followed by incubation with azophloxine. Sections were then incubated with tungstophosphoric acid orange $G$ solution and light green solution for collagen staining. Dehydration was performed by increasing concentrations of ethanol. Slides were incubated with HistoChoice clearing agent (Sigma-Aldrich, UK) and mounted with DPX (Fisher Scientific, UK).

\section{SDS PAGE and Immunoblotting}

Frozen muscles and liver were homogenised in RIPA lysis buffer containing 1\%(v/v) 4nonylphenyl-polyethylene glycol (Sigma- Aldrich, UK), 10\% (w/v) sodium dodecyl sulphate (SDS, Fisher Scientific, UK), 0.5\% (w/v) sodium deoxycholate (Fisher Scientific, UK) and 1XRoche Complete Protease Inhibitor Cocktail in PBS. $30 \mu \mathrm{g}$ of muscle or liver protein extracts were resolved in 10\% SDS-PAGE gels and transferred onto polyvinylidene difluoride membranes (Amersham, USA). Membranes were blocked for 60 minutes with 5\% $(w / v)$ milk dissolved in Tris-buffered-saline-Tween $(0.1 \%)$ and incubated with primary antibodies. Chemiluminescent substrate 1 containing final concentrations of $2.5 \mathrm{mM}$ luminol, $0.45 \mathrm{mM}$ P-Coumaric acid and $0.1 \mathrm{M}$ Tris was mixed in a 1:1 ratio with Chemiluminescent 
substrate 2 containing final concentrations of $0.1 \%(\mathrm{v} / \mathrm{v})$ hydrogen peroxide $\left(\mathrm{H}_{2} \mathrm{O}_{2}\right)$ and $0.1 \mathrm{M}$ Tris for detection of signal. Blots were stripped using buffer containing $1.5 \%(\mathrm{w} / \mathrm{v})$ SDS and glycine, $\mathrm{pH} 2$ and were re-probed if necessary. Details of antibodies are given in Supplementary Table 2. For detection of protein carbonyls the Oxidized Protein Western Blot Detection Kit (Abcam, UK) was used according to the manufacturer's instructions. 3-4 $\mu \mathrm{g}$ of muscle and liver protein lysates were denatured with 12\% SDS and either derivatised with 2,4-dinitrophenylhydrazine (DNPH) or treated with control solution for 15 minutes. The derivatisation was neutralised with addition of equal volume of neutralisation buffer. The derivatised samples and their controls were resolved in 10\% SDS-PAGE gels and transferred onto polyvinylidene difluoride membranes. Membranes were blocked for 60 minutes with $5 \%$ milk dissolved in PBS-Tween (0.05\%) and incubated with anti-DNP primary antibody (1:5000) overnight. Subsequently, membranes were incubated with HRP conjugated secondary antibody (1:5000) and signal was detected with chemiluminescence.

\section{Intramuscular lipid contents by gas chromatography}

Intramuscular lipids were analysed by a combination of thin-layer chromatography and gas chromatography. Lipids were extracted from muscle samples with chloroform-methanol 2:1 $(\mathrm{v} / \mathrm{v})$ in the presence of $0.005 \%(\mathrm{w} / \mathrm{v})$ butylated hydroxytoluene, as an antioxidant, and fixed amounts of triheptadecanoyl glycerol (Sigma, St. Louis, MO), as triacylglycerol internal standard, and diheptadecanoyl phosphatidyl choline (Larodan, Solna, Sweden), as phospholipid internal standard. An aliquot of each lipid extract was spotted on a highperformance silica gel plate (Macherey-Nagel, Düren, Germany), which was then developed in petroleum ether - diethyl ether - acetic acid 80:20:1 (v/v/v). Lipid spots were visualised under ultraviolet light after spraying the plate with $0.2 \%(\mathrm{w} / \mathrm{v})$ dichlorofluorescein in ethanol. The triacylglycerol and phospholipid spots were scraped off and transferred to screw-cap tubes. Fatty acid methyl esters (FAMEs) were produced by the addition of $1 \mathrm{ml}$ of methanol sulfuric acid $96: 4(\mathrm{v} / \mathrm{v})$ and heating at $64^{\circ} \mathrm{C}$ overnight. The FAMEs were then extracted with 
$1 \mathrm{ml}$ of hexane and were separated in an Agilent 7890A gas chromatograph (Santa Clara, CA), equipped with a 30 m-long AT-WAX capillary column (Alltech, Deerfield, IL) and flame ionization detector. The column temperature was programmed from $140^{\circ}$ to $270^{\circ} \mathrm{C}$ at $40^{\circ} \mathrm{C}$ $\min ^{-1}$ and the run was held at $270^{\circ} \mathrm{C}$ for $4 \mathrm{~min}$. The carrier gas was helium at a flow rate of $1.6 \mathrm{ml} \mathrm{min}{ }^{-1}$. FAMEs were quantified in the chromatograms obtained with the aid of the Agilent ChemStation software by comparing the area under their peaks to that of methyl heptadecanoate (derived from the internal standards).

\section{${ }^{1} \mathrm{H}$ NMR spectroscopy-based metabonomic analysis}

Aqueous metabolites were extracted from gastrocnemius muscle. $40-50 \mathrm{mg}$ of muscle tissue were snap frozen in liquid nitrogen and finely ground in $300 \mu \mathrm{L}$ of chloroform: methanol 2:1 (v/v) using a tissue lyser. The homogenate was combined with $300 \mu \mathrm{L}$ of water, vortexed and centrifuged $(13,000 \times \mathrm{g}$ for 10 minutes) to separate the aqueous (upper) and organic (lower) phases. The water and methanol from the aqueous phase was removed using a vacuum concentrator (SpeedVac) before reconstitution in $550 \mu \mathrm{L}$ of phosphate buffer $(\mathrm{pH}$ 7.4) in $\mathrm{D}_{2} \mathrm{O}$ containing $1 \mathrm{mM}$ of the internal standard, 3-(trimethylsilyl)-[2,2,3,3,-2H4]propionic acid (TSP).

For each sample, an one-dimensional NMR spectrum was acquired with water peak suppression using a standard pulse sequence (recycle delay (RD) $-90^{\circ}-t_{1}-90^{\circ}-t_{m}-90^{\circ}$-acquire free induction decay (FID). RD was set as $2 \mathrm{~s}$, the $90^{\circ}$ pulse length was $12.06 \mu \mathrm{s}$, and the mixing time (tm) was $10 \mathrm{~ms}$. For each spectrum, 4 dummy scans were followed by 64 scans with an acquisition time of $2.7 \mathrm{~s}$ per scan and collected in $64 \mathrm{~K}$ data points with a spectral width of $12.001 \mathrm{ppm} .{ }^{1} \mathrm{H}$ nuclear magnetic resonance (NMR) spectra were manually corrected for phase and baseline distortions and referenced to the TSP singlet at $\delta 0.0$. Spectra were digitised using an in-house MATLAB (version R20016a, The Mathworks, Inc.; Natwick, MA) script. To minimise baseline distortions arising from imperfect water saturation 
the region containing the water and TSP resonances was excised from the spectra. Principal components analysis (PCA) was performed with Pareto scaling in MATLAB using in-house scripts.

TBARS, protein carbonyls and phospho-MAPK Proteome Profiler Array

Thiobarbituric Acid Reactive Substances (TBARS) were evaluated with the OXItek TBARS Assay Kit (Enzo Life Sciences, USA) according to the manufacturer's instructions. The product of thiobarbituric acid plus malondialdehyde (MDA) was measured by fluorometry with excitation at $530 \mathrm{~nm}$ and emission at $590 \mathrm{~nm}$ in a TECAN Infinite M200 plate reader (Tecan, Switzerland). The TBARS values were normalised to protein content.

Protein carbonyl content was assayed in whole muscle and liver lysates with the Protein Carbonyl Content Assay Kit (Sigma-Aldrich, UK) according to the manufacturer's instructions. Muscle and liver lysates were treated with 10\% Streptozocin for 15 minutes followed by derivatisation with DNPH for 10 minutes. The derivatised proteins were precipitated with $20 \%$ trichloroacetic acid and the pellets were washed with acetone for removal of free DNPH prior to resolubilisation in $6 \mathrm{M}$ guanidine solution. Carbonyl content was measured spectrophotometrically at $375 \mathrm{~nm}$ in a TECAN Infinite M200 plate reader (Tecan, Switzerland). Results were calculated as nmol carbonyl/mg protein using an extinction coefficient of $22,000 \mathrm{M}^{-1} \mathrm{~cm}^{-1}$.

MAPK phosphorylation was determined with the human phospho-MAPK proteome profiler array according to the manufacturer's instructions (R\&D, UK). Pooled samples of three quadriceps muscles from the same experimental group were homogenised in lysis buffer and adjusted to $300 \mathrm{mg}$ protein per $300 \mathrm{~mL}$ lysate. Signals were detected with Chemiluminescent Substrate. 


\section{DHE staining and Liquid Chromatography Mass Spectrometry (LC-MS) detection of 2- hydroxyethidium}

Muscle and liver tissues were incubated with $5 \mu \mathrm{mol} / \mathrm{L}$ of dihydroethidium (DHE) in PBS, for 30 min at $37^{\circ} \mathrm{C}$ and were subsequently washed with PBS and mounted. For LC-MS analysis, muscle sections were stained with DHE and washed with PBSdiethylenetriaminepentaacetic acid (DTPA). Four volumes of methanol were added to the tissues followed by incubation at $-20^{\circ} \mathrm{C}$ overnight. The resulting lysates were centrifuged at $15,000 \times \mathrm{g}$ for 10 minutes at $4^{\circ} \mathrm{C}$. The supernatant was evaporated to dryness using a vacuum concentrator (Genevac MiVac, Genevac, Ipswich, UK), and the resulting residue was dissolved in 1:1 water:stabilisation solution $(33.3 \%$ methanol, $5 \mu \mathrm{moL} / \mathrm{L}$ final concentration fluorescein internal standard, $0.1 \mathrm{mmoL} / \mathrm{L}-\mathrm{DTPA}$, and $5 \mathrm{mmoL} / \mathrm{L}$ sodium ascorbate) with a final volume of $120 \mu \mathrm{L}$ for LC-MS analysis. The pellet was dissolved in 50 $\mu \mathrm{L}$ of sodium hydroxide $(0.1 \mathrm{M})$ and the protein content quantified using the Pierce ${ }^{\mathrm{TM}} \mathrm{BCA}$ Protein Assay Kit (ThermoFisher Scientific, USA) as directed by the manufacturer. For LCMS analysis of DHE oxidation products, the specific superoxide reaction product 2hydroxyethidium (2-OH-E+) was detected at an $\mathrm{m} / \mathrm{z}$ ratio of 330.3. Separation was achieved using a Shimadzu prominence LC20 quaternary pump and autosampler, a Shimadzu CTO10 column oven, and an Agilent Eclipse XDBMC18 column $(5 \mu \mathrm{m}, 4.6 \times 150 \mathrm{~mm})$ at a flow rate of $0.5 \mathrm{~mL} / \mathrm{min}$. Fluorescein was used as an internal standard, at an $\mathrm{m} / \mathrm{z}$ ratio of 333.0 and a retention time of 11.25 minutes. Peaks corresponding to 2-OH-E+ and fluorescein were integrated and peak areas were calculated. The ratio of $2-\mathrm{OH}-\mathrm{E}+$ to fluorescein was normalised to the total protein content. 


\section{Supplementary Tables}

Supplementary Table 1. Primary and secondary antibodies for immunofluorescence

\begin{tabular}{llll}
\hline Antigen & Species & Dilution & Supplier, Cat Number \\
\hline MHCI & Mouse & $1: 1$ & DSHB A4.840 \\
MHCIIA & Mouse & $1: 200$ & DSHB A4.74 \\
MHCIIB & Mouse & $1: 1$ & DSHB BF.F3 \\
Laminin & Rabbit & $1: 400$ & Sigma L9393 \\
CD31 & Rat & $1: 40$ & AbD serotec MCA2388 \\
F4.80 & Rat & $1: 100$ & Bio-RAD MCA4978 \\
CD36 & Rabbit & $1: 100$ & Novus Biologicals NB400-144 \\
3NT & Mouse & $1: 500$ & Santa Cruz 32757 \\
8-OHdG & Mouse & $1: 100$ & Santa Cruz 393871 \\
E06 & Mouse & $1: 100$ & Avanti lipids 330002S \\
Alexa fluor 633 anti-mouse & Goat & $1: 200$ & Life Technologies A20146 \\
Alexa fluor 488 anti-mouse & Goat & $1: 200$ & Life Technologies A11029 \\
Alexa fluor 488 anti-rabbit & Goat & $1: 200$ & Life Technologies A11034 \\
Alexa fluor 594 anti-rabbit & Goat & $1: 200$ & Life Technologies A11037 \\
\hline
\end{tabular}

Supplementary Table 2. Primary and secondary antibodies for immunoblotting

\begin{tabular}{llll}
\hline Antigen & Species & Dilution & Supplier, Cat Number \\
\hline 3NT & Mouse & $1: 200$ & Cayman 189542 \\
4HNE & Mouse & $1: 1,000$ & R\&D Systems MAB3249 \\
p-p38 & Mouse & $1: 1000$ & Santa Cruz 166182 \\
p38 & Mouse & $1: 800$ & Santa Cruz 7972 \\
$\beta$ tubulin & Mouse & $1: 1,000$ & EMD Millipore 05-661 \\
HRP Conjugated anti-mouse & Goat & $1: 10,000$ & Sigma A9044 \\
\hline
\end{tabular}


Supplementary Table 3. qPCR primers sequence

\begin{tabular}{lll}
\hline $\begin{array}{l}\text { Oligo } \\
\text { Name }\end{array}$ & Forward & Reverse \\
\hline Catalase & GGATTATGGCCTCCGAGATCTT & TAAAACGTCCAGGACGGGTAA \\
\hline Cd36 & AGATGACGTGGCAAAGAACAG & CCTTGGCTAGATAACGAACTCTG \\
\hline Cidea & CATGGTTTGAACTCGAAAAGG & TGACATTCATGGGATTGCAGAC \\
\hline Fatp1 & TGGAGAGCACCAAGACAGACA & TGCCGGAGTCGACAATGAT \\
\hline Fgf1 & GAAGCATGCGGAGAAGAACTG & CGAGGACCGCGCTTACAG \\
\hline F4.80 & CTGCACCTGTAAACGAGGCTT & GCAGACTGAGTTAGGACCACAA \\
\hline Gpx1 & AATGTCGCGTCTCTCTGAGG & TCCGAACTGATTGCACGGG \\
\hline Gpx4 & GCCTGGATAAGTACAGGGGTT & CATGCAGATCGACTAGCTGAG \\
\hline Had & GCTGGGCCTAACTTTGAGTATG & CAAAATCAGCGTCATCAGGAGAA \\
\hline Hprt & GCTCGAGATGTCATGAAGGAGAT & AAAGAACTTATAGCCCCCCTTGA \\
\hline LPL & GCTGGGCCTAACTTTGAGTATG & CAAAATCAGCGTCATCAGGAGAA \\
\hline Mcp1 & TAAAAACCTGGATCGGAACCAA & GCATTAGCTTCAGATTTACGGGT \\
\hline Mgst1 & CCTCCTATGCAACGATCATTCTT & ACCTTGTTGGTTATCCCTCTGG \\
\hline Nox2 & TGAATGCCAGAGTCGGGATT & CGAGTCACGGCCACATACA \\
\hline Ogg1 & CAACAACATTGCTCGCATTACTG & TCAAGCTGAATGAGTCGAGGT \\
\hline Parp1 & GGTCTTTAAGAGCGACGCTTAT & TTCTGTGTCTTGACCATGCAC \\
\hline Prdx1 & CTGGCATGGATTAACACACCC & GGTGCGCTTGGGATCTGAT \\
\hline Scd1 & TTCTTGCGATACACTCTGGTGC & CGGGATTGAATGTTCTTGTCGT \\
\hline Sod1 & TATGGGGACAATACACAAGGCT & CGGGCCACCATGTTTCTTAGA \\
\hline Sod2 & TGGACAAACCTGAGCCCTAAG & CCCAAAGTCACGCTTGATAGC \\
\hline Tnfa & CAGGCGGTGCCTATGTCTC & CGATCACCCCGAATTCAGTAG \\
\hline Vegfa165 & TGCAGGCTGCTGTAACGATG & GAACAAGGCTCACAGTGATTTTCT \\
\hline & & \\
\hline
\end{tabular}




\section{Supplementary Table 4. ApoE deficiency and Western-type diet independently alter intramuscular triacylglycerol distribution.}

\begin{tabular}{|c|c|c|c|c|c|}
\hline & \multicolumn{2}{|c|}{ Wild type } & \multicolumn{2}{|c|}{$\mathrm{ApoE}^{-/-}$} \\
\hline & & Normal Diet & Western Diet & Normal Diet & Western Diet \\
\hline $12: 0$ & Lauric acid & $0.0 \pm 0.0$ & $0.0 \pm 0.0$ & $0.0 \pm 0.0$ & $0.98 \pm 0.19^{a, b, c}$ \\
\hline $14: 0$ & Myristic acid & $1.51 \pm 1.39$ & $5.47 \pm 3.11$ & $1.91 \pm 2.34$ & $6.8 \pm 0.63^{a, b}$ \\
\hline $14: 1 n-9$ & $\begin{array}{l}\text { Myristoleic } \\
\text { acid }\end{array}$ & $0.0 \pm 0.0$ & $0.0 \pm 0.0$ & $0.0 \pm 0.0$ & $1.13 \pm 0.18^{a, b, c}$ \\
\hline 15:0 & $\begin{array}{l}\text { Pentadecano } \\
\text { ic acid }\end{array}$ & $0.0 \pm 0.0$ & $0.56 \pm 0.51$ & $0.3 \pm 0.32$ & $0.93 \pm 0.07^{\mathrm{a}, \mathrm{b}}$ \\
\hline 16:0 & Palmitic acid & $25.31 \pm 2.78$ & $28.39 \pm 3.00$ & $23.54 \pm 2.24$ & $23.56 \pm 1.57$ \\
\hline $16: 1 n-7$ & $\begin{array}{l}\text { Palmitoleic } \\
\text { acid }\end{array}$ & $11.96 \pm 4.19$ & $18.11 \pm 2.03^{a}$ & $13.74 \pm 3.62$ & $17.86 \pm 1.31^{a}$ \\
\hline $18: 0$ & Stearic acid & $6.68 \pm 2.14$ & $2.27 \pm 0.17^{a}$ & $2.68 \pm 1.82^{a}$ & $1.81 \pm 0.25^{a}$ \\
\hline $18: 1 n-9$ & Oleic acid & $29.22 \pm 1.37$ & $37.04 \pm 1.97^{a}$ & $37.37 \pm 1.79^{a}$ & $38.48 \pm 1.49^{a}$ \\
\hline $18: 1 n-7$ & $\begin{array}{l}\text { cis-Vaccenic } \\
\text { acid }\end{array}$ & $2.63 \pm 1.57$ & $2.69 \pm 0.44$ & $3.74 \pm 1.15$ & $1.77 \pm 1.01$ \\
\hline $18: 2 n-6$ & Linoleic acid & $19.09 \pm 2.82$ & $5.19 \pm 0.73^{a}$ & $17.71 \pm 2.04$ & $6.11 \pm 0.77^{\mathrm{a}, \mathrm{b}}$ \\
\hline $18: 3 n-3$ & $\begin{array}{l}\text { a-Linolenic } \\
\text { acid }\end{array}$ & $3.60 \pm 1.47$ & $0.25 \pm 0.34^{a}$ & $0.40 \pm 0.40^{a}$ & $0.58 \pm 0.10^{a}$ \\
\hline \multirow[t]{4}{*}{$20: 3 n-3$} & $\begin{array}{l}\text { Dihomo- } \alpha- \\
\text { linolenic acid }\end{array}$ & $0.00 \pm 0.00$ & $0.04 \pm 0.09$ & $0.19 \pm 0.18$ & $0.00 \pm 0.00$ \\
\hline & SFA & $26.82 \pm 2.77$ & $34.42 \pm 4.37^{a, b}$ & $25.75 \pm 3.14$ & $32.26 \pm 2.24^{\mathrm{a}, \mathrm{b}}$ \\
\hline & MUFA & $42.87 \pm 3.27$ & $57.84 \pm 4.28^{a}$ & $54.85 \pm 3.65^{\mathrm{a}}$ & $59.24 \pm 1.99^{a}$ \\
\hline & PUFA & $23.50 \pm 2.09$ & $5.47 \pm 0.98^{a, b}$ & $16.71 \pm 3.90^{\mathrm{a}}$ & $6.69 \pm 0.76^{a, b}$ \\
\hline
\end{tabular}

Fatty acid distribution of skeletal muscle triacylglycerols was assessed in C57BI/6J (wild type, WT) and $\mathrm{ApoE}^{--}$mice fed a standard chow diet (normal diet, ND) or Western-type diet (WD) for 12 weeks. Data are expressed as mean \pm SD of percentage of fatty acids as well as percentage of total saturated fatty acids (SFA), total monounsaturated fatty acids (MUFA) and total polyunsaturated fatty acids (PUFA) in intramuscular triacylglycerols. Statistical analysis was performed by two-way ANOVA (Main effects: Genotype: $p<0.05$ for lauric, myristoleic, pentadecanoic, stearic, oleic and $\alpha$-linolenic acids, MUFA and PUFA; Diet $p<0.01$ for lauric, myristic, myristoleic, pentadecanoic, palmitoleic, stearic, oleic, linoleic and $\alpha$-linolenic acids, SFA, MUFA and PUFA; Interaction; $p<0.05$ for lauric, myristoleic, stearic, oleic and $\alpha$-linolenic acids, MUFA and PUFA) followed by Bonferroni post hoc tests; $a$,

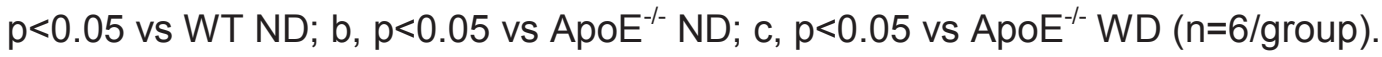


Supplementary Table 5. ApoE deficiency and Western-type diet independently alter intramuscular phospholipid distribution.

Wild type

$\mathrm{ApoE}^{-1-}$

Normal Diet Western Diet Normal Diet Western Diet

\begin{tabular}{|c|c|c|c|c|c|}
\hline 14:0 & Myristic acid & $0.91 \pm 0.05$ & $2.56 \pm 0.18^{a, b}$ & $1.82 \pm 0.39^{a}$ & $2.72 \pm 0.34^{a, b}$ \\
\hline 15:0 & $\begin{array}{l}\text { Pentadecano } \\
\text { ic acid }\end{array}$ & $0.32 \pm 0.06$ & $0.66 \pm 0.05$ & $0.47 \pm 0.10$ & $0.77 \pm 0.12^{\mathrm{a}, \mathrm{b}}$ \\
\hline $16: 0$ & Palmitic acid & $33.4 \pm 2.9$ & $33.3 \pm 1.3$ & $32.5 \pm 1.8$ & $34.4 \pm 5.6$ \\
\hline $16: 1 n-7$ & $\begin{array}{l}\text { Palmitoleic } \\
\text { acid }\end{array}$ & $1.7 \pm 0.23$ & $3.2 \pm 0.22^{a, b}$ & $2.4 \pm 0.34$ & $2.6 \pm 0.40^{a, b}$ \\
\hline 18:0 & Stearic acid & $17.29 \pm 0.94$ & $17.88 \pm 1.46$ & $18.67 \pm 2.20$ & $17.94 \pm 2.69$ \\
\hline $18: 1 n-9$ & Oleic acid & $5.11 \pm 0.32$ & $9.15 \pm 1.07^{a}$ & $7.78 \pm 2.28^{a}$ & $8.04 \pm 1.10^{\mathrm{a}}$ \\
\hline $18: 1 n-7$ & $\begin{array}{l}\text { cis-Vaccenic } \\
\text { acid }\end{array}$ & $3.46 \pm 0.17$ & $4.40 \pm 0.26^{a, b, c}$ & $3.90 \pm 0.21$ & $3.23 \pm 0.18$ \\
\hline $18: 2 n-6$ & Linoleic acid & $10.8 \pm 0.58$ & $8.7 \pm 0.70$ & $11.0 \pm 1.79^{a}$ & $10.3 \pm 2.12$ \\
\hline $18: 3 n-3$ & $\begin{array}{l}\alpha \text {-Linolenic } \\
\text { acid }\end{array}$ & $0.60 \pm 0.1$ & $0.28 \pm 0.1^{\mathrm{a}}$ & $0.34 \pm 0.1^{\mathrm{a}}$ & $0.27 \pm 0.02^{a}$ \\
\hline $20: 3 n-6$ & $\begin{array}{l}\text { Dihomo-Y- } \\
\text { linolenic acid }\end{array}$ & $0.53 \pm 0.3$ & $1.55 \pm 0.08^{a, b}$ & $0.84 \pm 0.64$ & $1.59 \pm 0.39^{a, b}$ \\
\hline $20: 3 n-3$ & $\begin{array}{l}\text { Dihomo- } \alpha- \\
\text { linolenic acid }\end{array}$ & $8.95 \pm 0.78$ & $8.43 \pm 0.88$ & $8.24 \pm 1.47$ & $8.0 \pm 1.94$ \\
\hline $22: 4 n-6$ & $\begin{array}{l}\text { Docosatetrae } \\
\text { noic acid }\end{array}$ & $0.77 \pm 0.12$ & $0.42 \pm 0.07^{\mathrm{a}}$ & $0.50 \pm 0.16^{a}$ & $0.29 \pm 0.16^{a}$ \\
\hline $22: 5 n-3$ & $\begin{array}{l}\text { Docosapenta } \\
\text { enoic acid }\end{array}$ & $1.14 \pm 0.43$ & $0.74 \pm 0.27$ & $0.85 \pm 0.26$ & $0.58 \pm 0.26$ \\
\hline $22: 6 n-3$ & $\begin{array}{l}\text { Docosahexa } \\
\text { enoic acid }\end{array}$ & $12.55 \pm 1.50$ & $7.23 \pm 1.08^{a}$ & $9.18 \pm 2.53$ & $7.33 \pm 2.14^{a}$ \\
\hline \multirow[t]{4}{*}{$24: 0$} & $\begin{array}{l}\text { Lignoceric } \\
\text { acid }\end{array}$ & $1.19 \pm 0.67$ & $1.61 \pm 0.20$ & $1.20 \pm 0.49$ & $2.03 \pm 0.52$ \\
\hline & SFA & $53.14 \pm 4.01$ & $55.84 \pm 2.28$ & $54.63 \pm 3.40$ & $54.33 \pm 2.43$ \\
\hline & MUFA & $10.29 \pm 0.61$ & $16.75 \pm 0.93^{\mathrm{a}, \mathrm{b}}$ & $14.06 \pm 2.25^{a}$ & $14.45 \pm 0.67^{a}$ \\
\hline & PUFA & $36.58 \pm 4.15$ & $27.42 \pm 2.67^{a}$ & $31.31 \pm 5.53$ & $31.22 \pm 2.39$ \\
\hline
\end{tabular}

Fatty acid distribution of skeletal muscle phospholipids was assessed in C57BI/6J (wild type, WT) and $\mathrm{ApoE}^{-/-}$mice fed a standard chow diet (normal diet, ND) or Western-type diet (WD) for 12 weeks. Data are expressed as mean \pm SD of percentage of fatty acids as well as percentage of total saturated fatty acids (SFA), total monounsaturated fatty acids (MUFA) and total polyunsaturated fatty acids (PUFA) in intramuscular phospholipids. Statistical analysis was performed by two-way ANOVA (Main effects: Genotype: $p<0.05$ for myristic, pentadecanoic, palmitoleic, cis-vaccenic and $\alpha$-linolenic acids, MUFA and PUFA; Diet 
$p<0.05$ for myristic, pentadecanoic, palmitoleic, oleic, linoleic, $\alpha$-linolenic, dihomo-y-linolenic, docosatetraenoic and docosahexaenoic acids, SFA, MUFA and PUFA; Interaction; $p<0.05$ for myristic, oleic and cis-vaccenic acids, MUFA and PUFA) followed by Bonferroni post hoc tests; $a, p<0.05$ vs WT ND; $b, p<0.05$ vs $\mathrm{ApoE}^{-/-} \mathrm{ND}$; $c, p<0.05$ vs $\mathrm{ApoE}^{-/-}$WD (n=6/group). 


\section{Supplementary figures}

A

AT ND

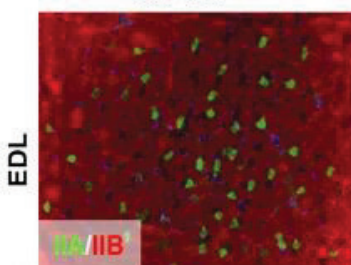

몀
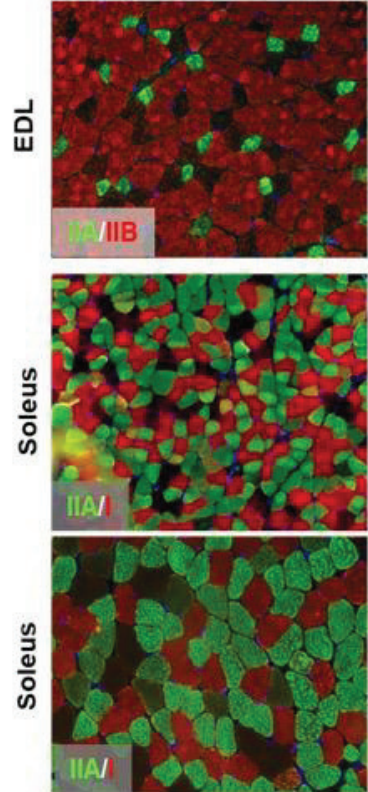

B

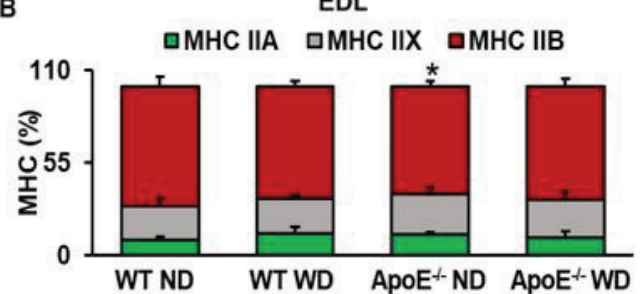

WT WD
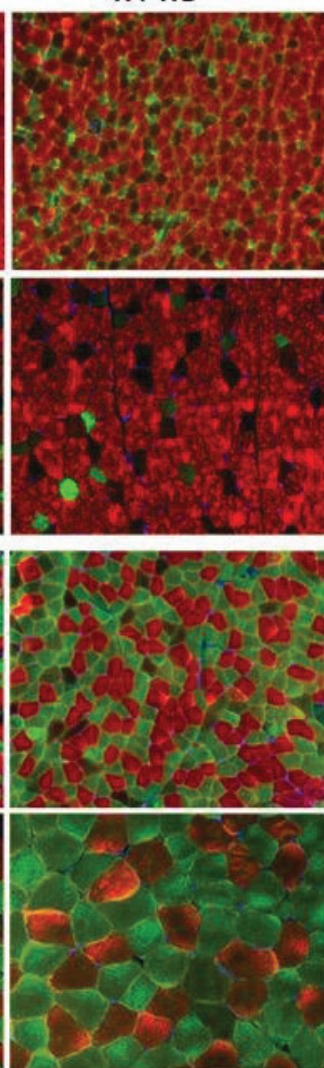

EDL
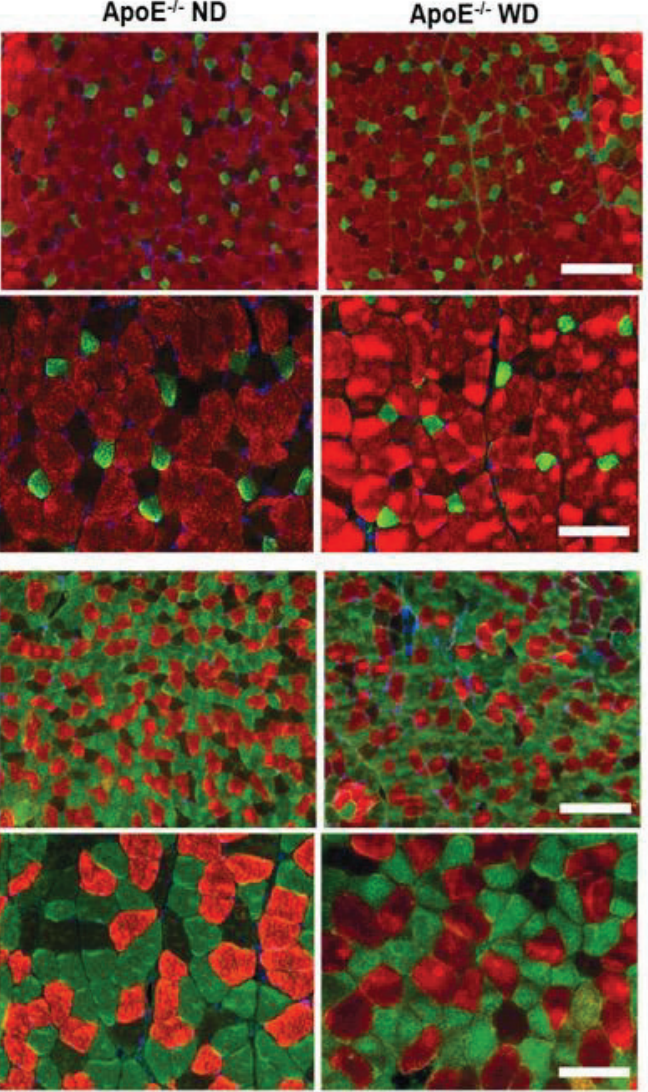

Soleus

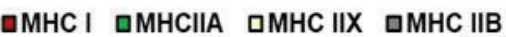

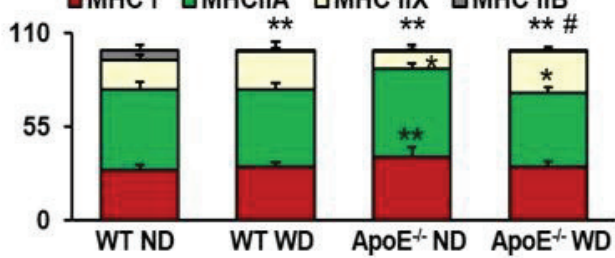

c

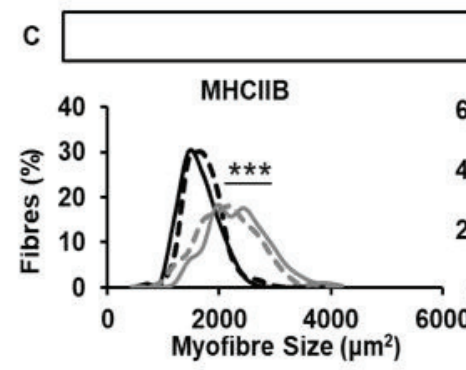

EDL
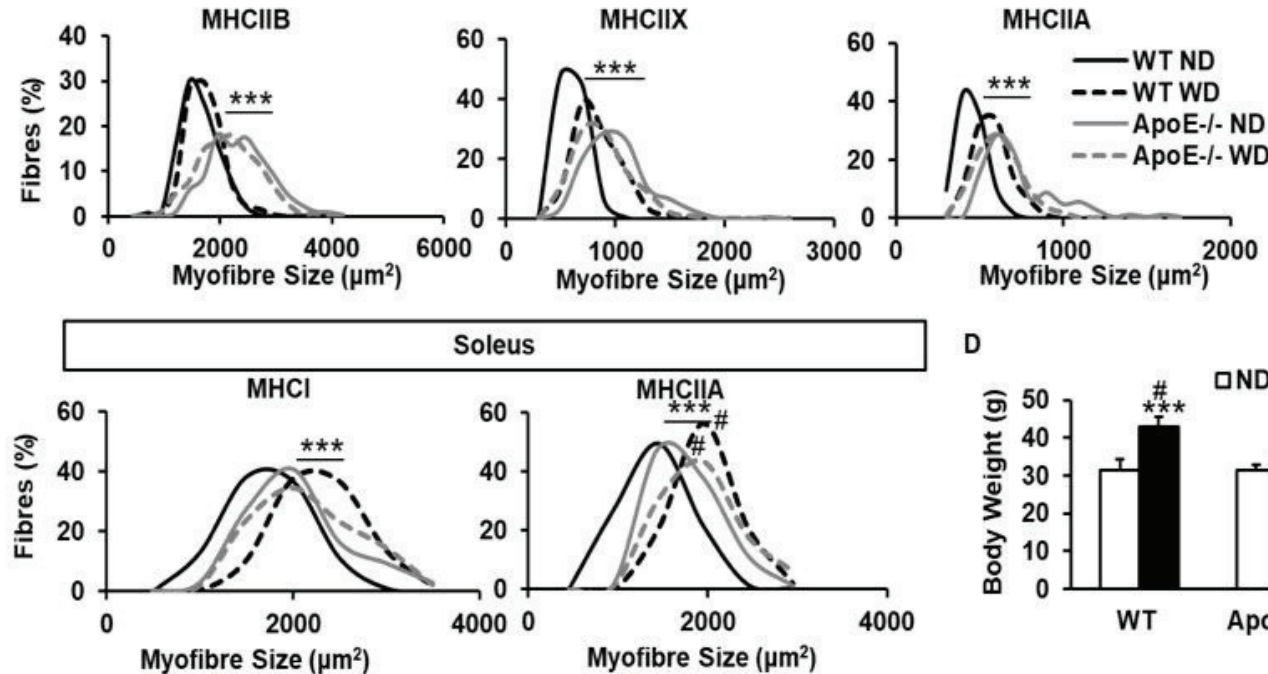

Soleus

D
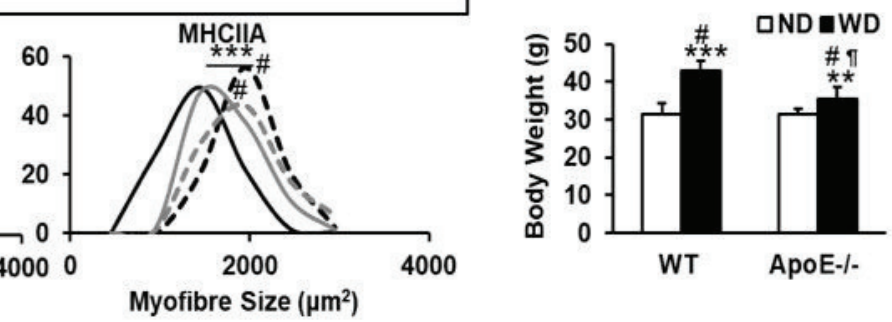
Supplementary Fig. 1. ApoE deficiency and Western-type diet induce a mild transition to slower myofibres. Myofibre composition and mitochondrial activity in EDL and soleus was assessed in C57BI/6J (wild-type, WT) and $\mathrm{ApoE}^{-/-}$mice fed a standard chow diet (normal diet, ND) or Western-type diet (WD) for 12 weeks. (A) Representative images of MHC immunostaining in EDL and soleus (x10 magnification, scale bar 200 $\mu m)$. (B) Quantification of myofibre type in EDL and soleus. Data are expressed as mean \pm SD. (C) Quantification of myofibre size in EDL and soleus. Data are expressed as frequency distribution. (D) Body weight of mice at the age of 20 weeks on either ND or a WD. Data are expressed as mean \pm SD. Statistical analysis was performed by two-way ANOVA for myofibre type and body weight (Main effects: Genotype: $p<0.05$ for MHCIIB in both soleus and EDL, MHCIIX in EDL, $\mathrm{MHCl}$ in soleus and body weight; Diet $\mathrm{p} \leq 0.01$ for MHCIIB, MHCIIX and MHCIIA in soleus and body weight; Interaction; $p<0.05$ for MHCIIB, MHCIIX, MHCI in soleus, MHCIIB and MHCIIA in EDL and body weight) followed by Bonferroni post hoc tests for myofibre type with ${ }^{*} p<0.05,{ }^{* *} p<0.01$, ${ }^{* * *} p<0.001$ vs WT ND, \#<0.05 vs $\mathrm{ApoE}^{-/-} \mathrm{ND}$ and $\mathbb{\pi}<0.05$ vs WT WD ( $\mathrm{n}=6 /$ group). Statistical analysis for myofibre size was performed by $X^{2}$ square ( $\left.n=6 / g r o u p\right)$. For EDL: WT WD versus WT ND: $x^{2}=8.561 ; \mathrm{df}=9 ; \mathrm{p}=0.479$ for type IIB, $x^{2}=59.546 ; \mathrm{df}=7 ; p<0.001$ for type IIX and $X^{2}=53.982 ; \mathrm{df}=6 ; p<0.001$ for type IIA. ApoE ${ }^{-/-}$ND versus WT ND: $X^{2}=86.338 ; \mathrm{df}=14 ; p<0.001$ for type IIB, $X^{2}=127.109 ; \mathrm{df}=10 ; p<0.001$ for type IIX and $X^{2}=118.243 ; \mathrm{df}=4 ; \mathrm{p}<0.001$ for type IIA. For $\mathrm{ApoE}^{-/}$WD versus WT ND: $X^{2}=37.620 ; \mathrm{df}=9 ; \mathrm{p}<0.001$ for type IIB, $X^{2}=19300 ; \mathrm{df}=10 ; \mathrm{p}<0.001$ for type IIX and $X^{2}=11.000 ; \mathrm{df}=6 ; \mathrm{p}<0.001$ for type IIA. Moreover for $\mathrm{ApoE}^{-/-}$WD versus $\mathrm{ApoE}^{-/-} \mathrm{ND}$ : $X^{2}=9.293 ; \mathrm{df}=9 ; \mathrm{p}=0.812$ for type IIB, $X^{2}=26.301 ; \mathrm{df}=10 ; \mathrm{p}<0.05$ for type IIX and $X^{2}=27.012 ; \mathrm{df}=14 ; \mathrm{p}<0.05$ for type IIA. For soleus, WT WD versus WT ND: $X^{2}=56.683 ; \mathrm{df}=5 ; \mathrm{p}<0.001$ for type $\mathrm{I}$ and $X^{2}=71.358 ; \mathrm{df}=4 ; \mathrm{p}<0.001$ for type IIA. ApoE ${ }^{-/-} \mathrm{ND}$ versus WT ND: $X^{2}=23.787 ; \mathrm{df}=5 ; \mathrm{p}<0.001$ for type $\mathrm{I}$ and $\chi^{2}=33.788 ; \mathrm{df}=4 ; \mathrm{p}<0.001$ for type IIA. ApoE ${ }^{-l-}$ WD versus WT ND: $X^{2}=200.000 ; \mathrm{df}=4 ; p<0.001$ for type $\mathrm{I}$ and $\chi^{2}=28.000 ; \mathrm{df}=5$, $p<0.001$ for type IIA. ApoE ${ }^{-/-}$WD versus $A p o E^{-/-} N D: X^{2}=6.663 ; d f=4 ; p=0.068$ for type I and $x^{2}=9.692 ; \mathrm{df}=4 ; \mathrm{p}=0.046$ for type IIA. 
A WT ND
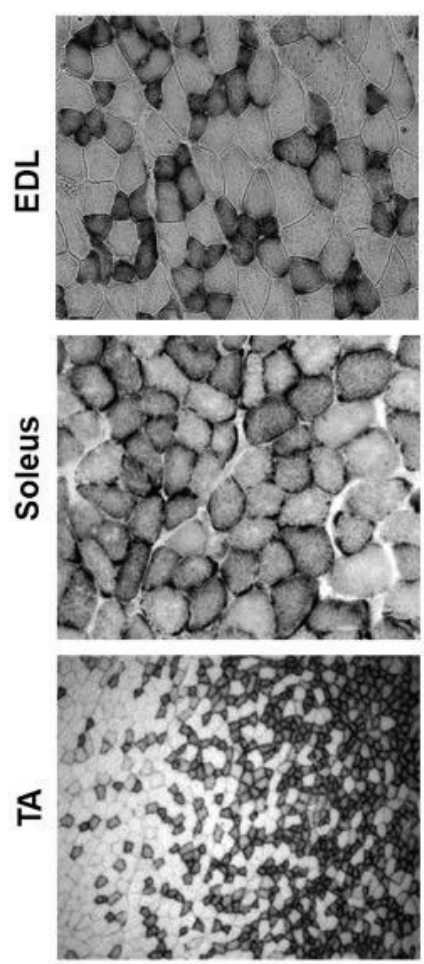

B
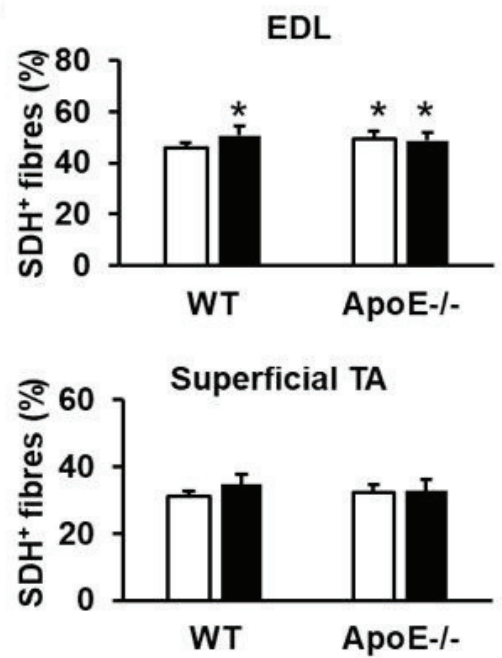

WT WD
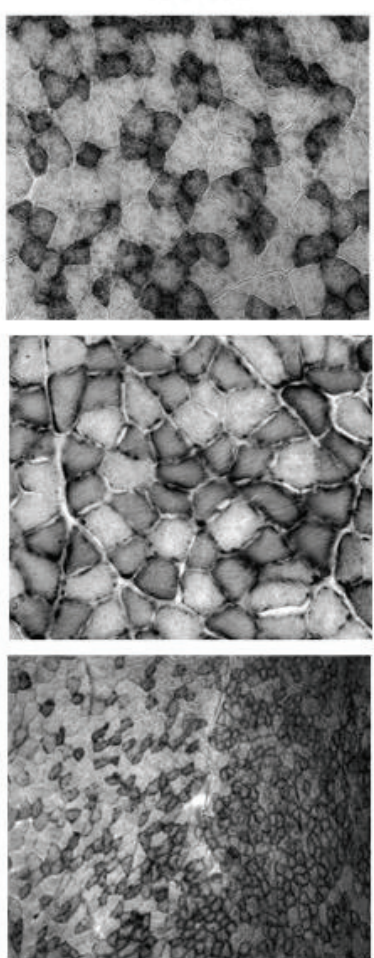

ApoE $^{-/-}$ND
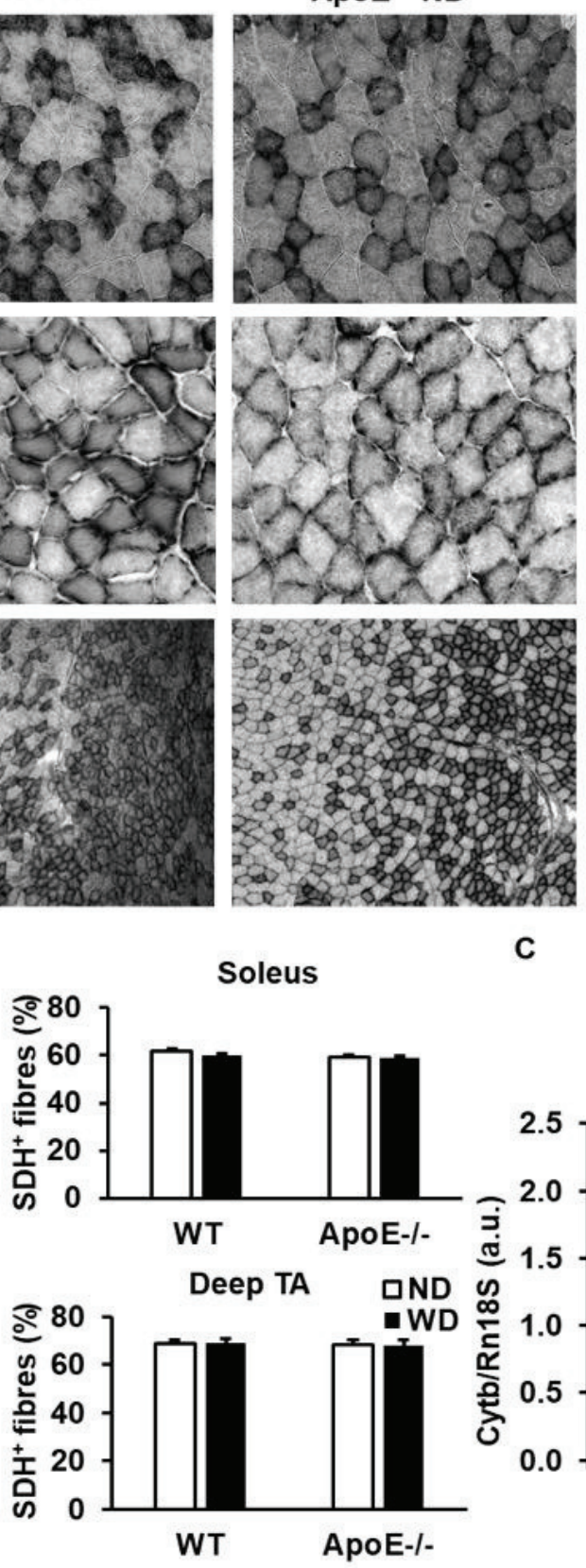

C

ApoE $^{-/-}$WD
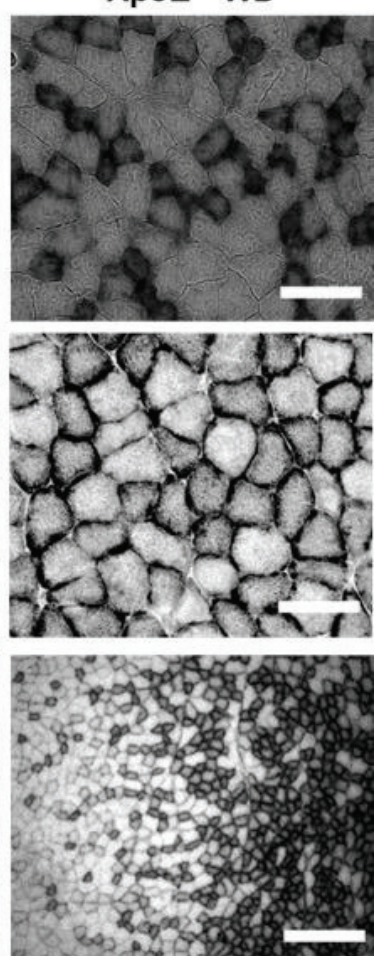

Mitochondrial

Biogenesis

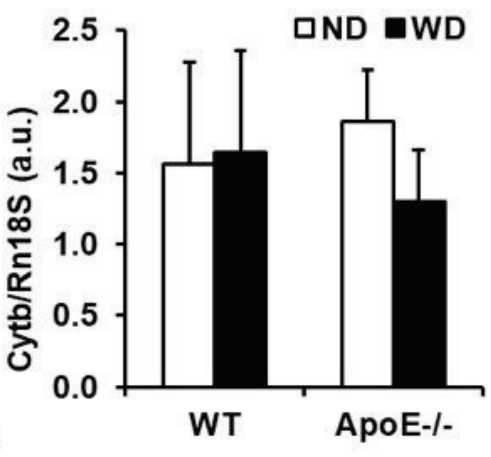

Supplementary Fig. 2. ApoE deficiency and Western-type diet do not affect mitochondrial activity or biogenesis. Myofibre composition and mitochondrial activity in skeletal muscle was assessed in C57BI/6 (wild-type, WT) and $\mathrm{ApoE}^{-/-}$mice fed a standard chow diet (normal diet, ND) or Western-type diet (WD) for 12 weeks. (A) Representative images of SDH staining in EDL, soleus and TA (superficial and deep) (x20 magnification, scale bar $100 \mu \mathrm{m}$ for EDL and soleus and x5 magnification, scale bar $400 \mu \mathrm{m}$ for TA). (B) Quantification of SDH staining in EDL, soleus and TA (superficial and deep). Data are expressed as mean \pm SD. (C) Ratio of mitochondrial to nuclear gene content for mitochondrial biogenesis in the gastrocnemius muscle. Data are expressed as mean \pm SD. Statistical analysis was performed by two-way ANOVA (Main effects: Diet: 
$p<0.05$; Interaction; $p<0.001$ for SDH staining in the EDL) followed by Bonferroni post hoc tests, ${ }^{*} p<0.05$ vs WT ND ( $\left.n=6 / g r o u p\right)$. 
A

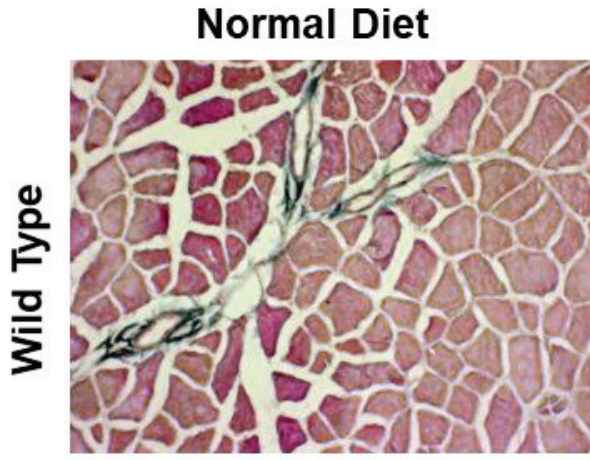

Western Diet
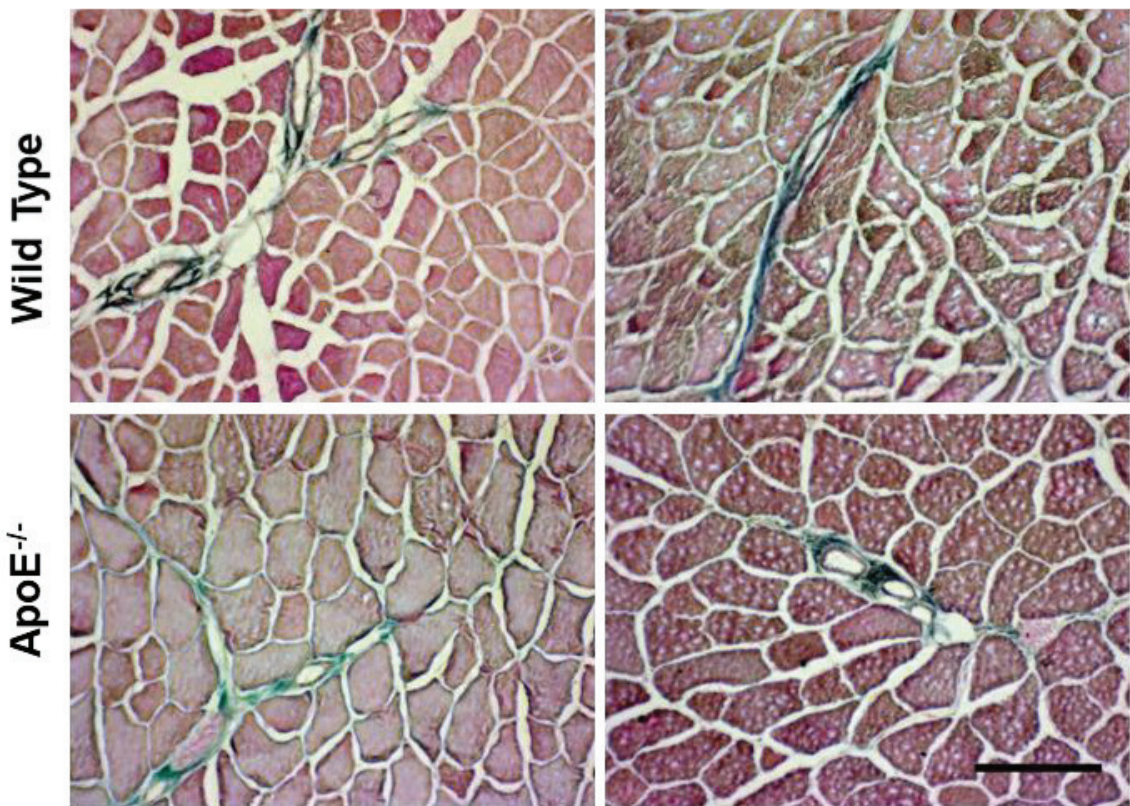

B

Normal Diet
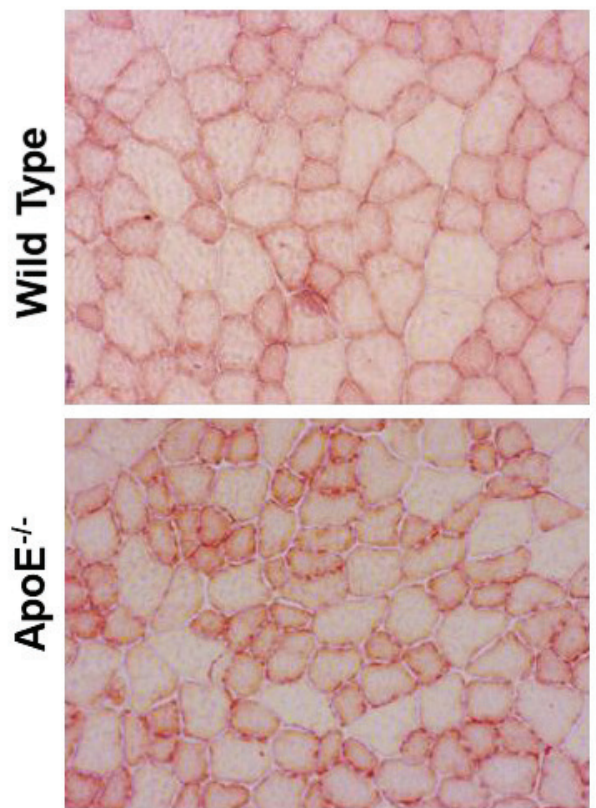

C

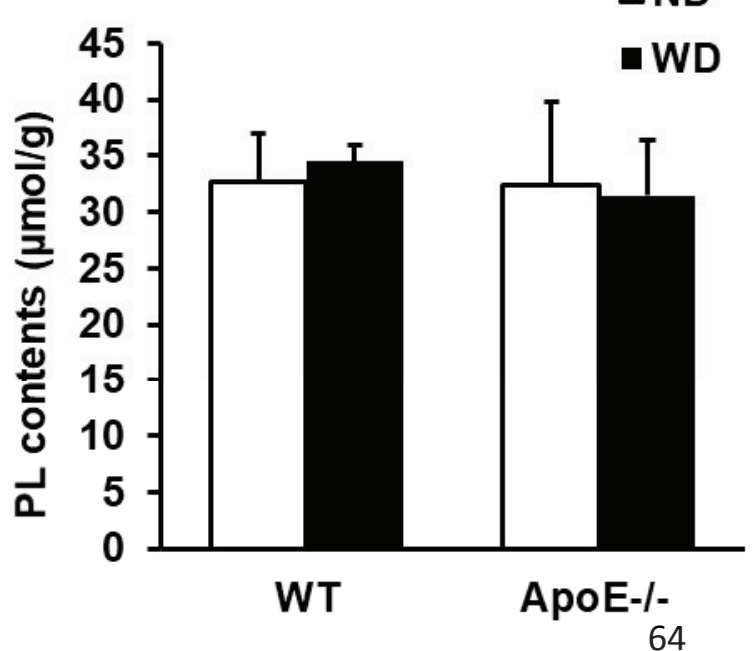

口ND

Western Diet
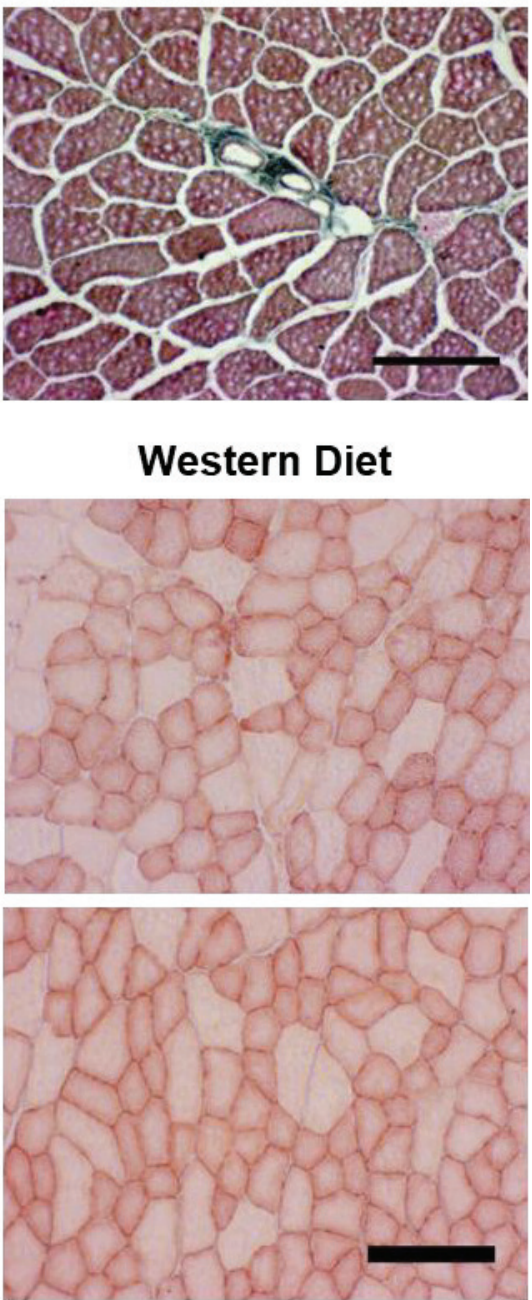
. 
Supplementary Fig. 3. ApoE deficiency and Western-type diet increase intramyocellular lipid content but do not induce fibrosis and do not alter phospholipid contents. Fibrosis, intramyocellular lipid and phospholipid contents was assessed in skeletal muscle of C57BI/6J (wild-type, WT) and $\mathrm{ApoE}^{-/-}$mice fed a standard chow diet (normal diet, ND) or Western-type diet (WD) for 12 weeks. (A) Representative images of Masson's Trichrome staining in TA (x20 magnification, scale bar $100 \mu \mathrm{m}$ ). (B) Representative images of Oil Red $\mathrm{O}$ staining in tibialis anterior ( $\mathrm{x} 20$ magnification, scale bar $50 \mu \mathrm{m})$. (C) Phospholipid content in tibialis anterior as determined by gas chromatography. Data are expressed as mean \pm SD. Statistical analysis was performed by two-way ANOVA ( $n=6 / g r o u p)$. 

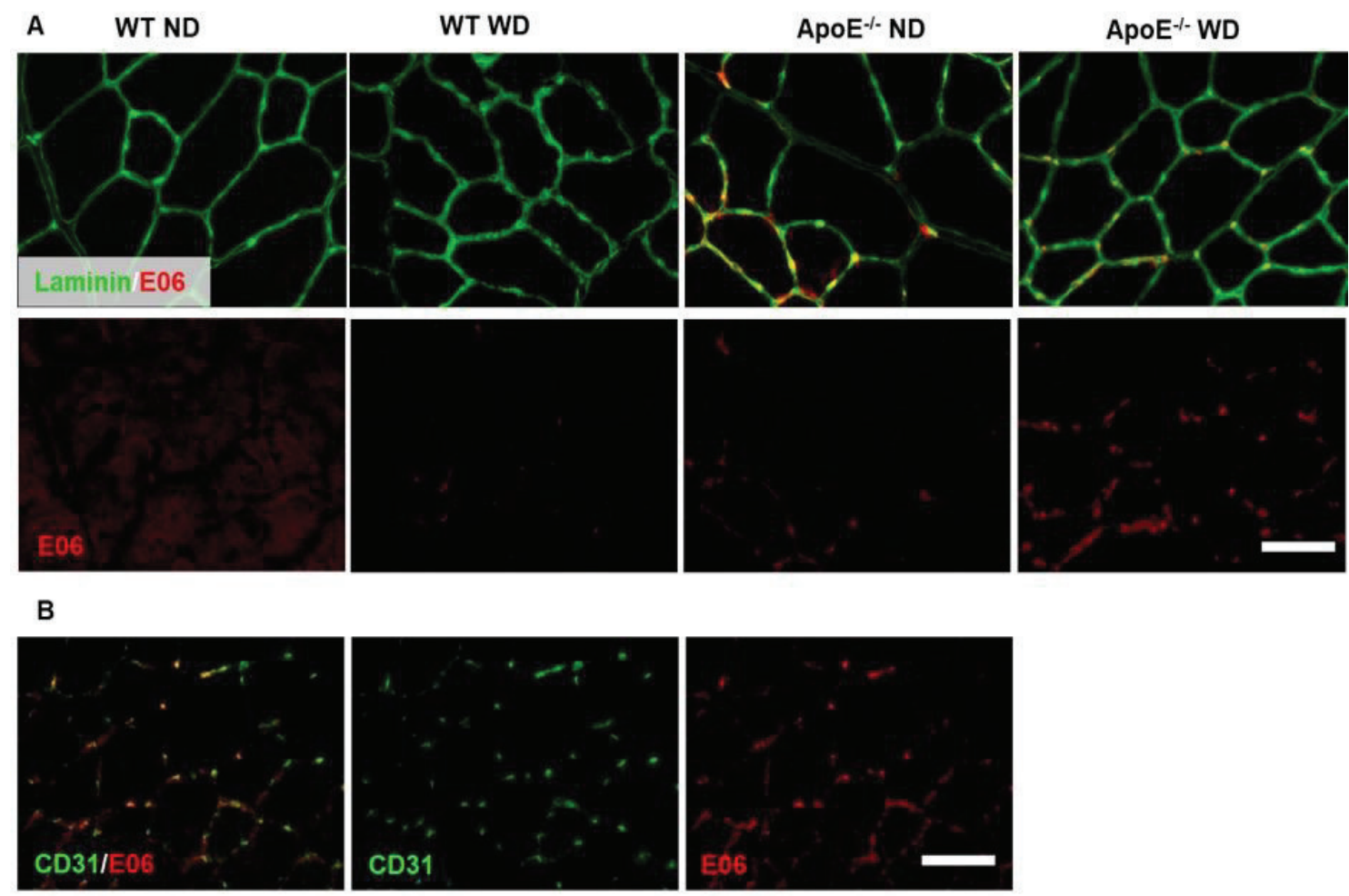

Supplementary Fig. 4. ApoE deficiency with Western-type diet increase oxidised phospholipids in capillaries of skeletal muscle. Oxidised phospholipid content was assessed in skeletal muscle of C57BI/6J (wild-type, WT) and $\mathrm{ApoE}^{-/-}$mice fed a standard chow diet (normal diet, ND) or Western-type diet (WD) for 12 weeks. (A) Representative images of E06 and laminin staining in tibialis anterior ( $x 40$ magnification, scale bar 50 $\mu \mathrm{m})$. (B) Representative images of E06 and CD31 staining, CD31 staining and E06 staining only in tibialis anterior (x40 magnification, scale bar $50 \mu \mathrm{m}$ ). 
A

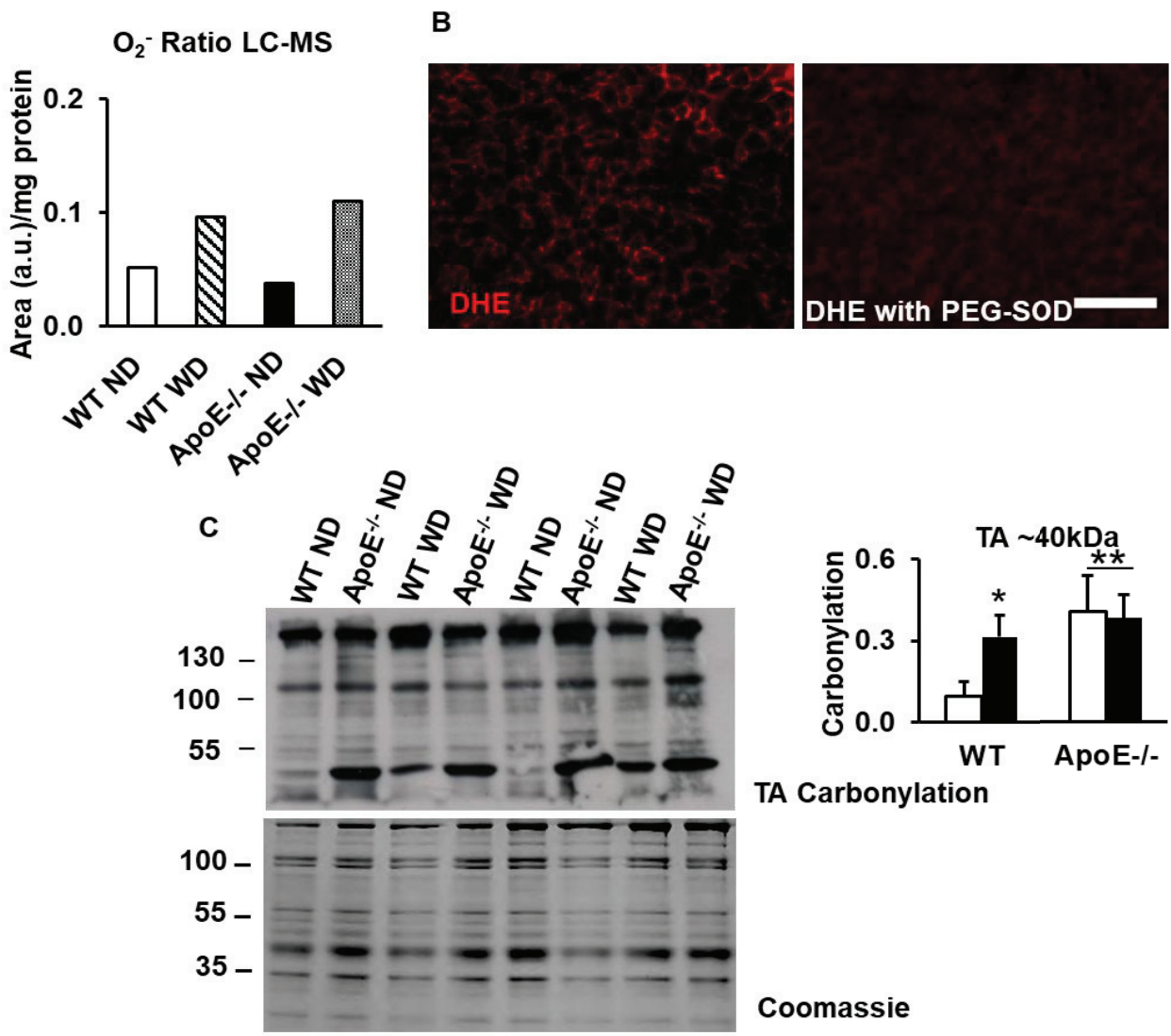

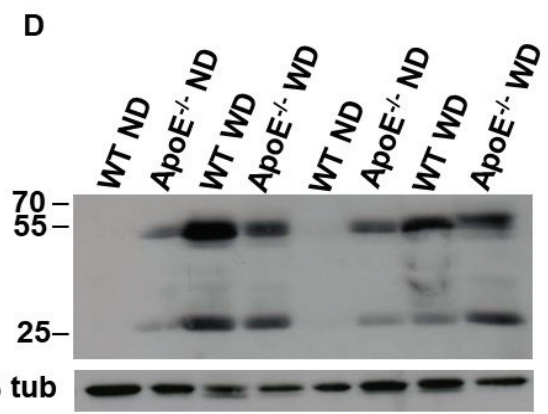

Tibialis anterior $3 \mathrm{NT}$
WT ND
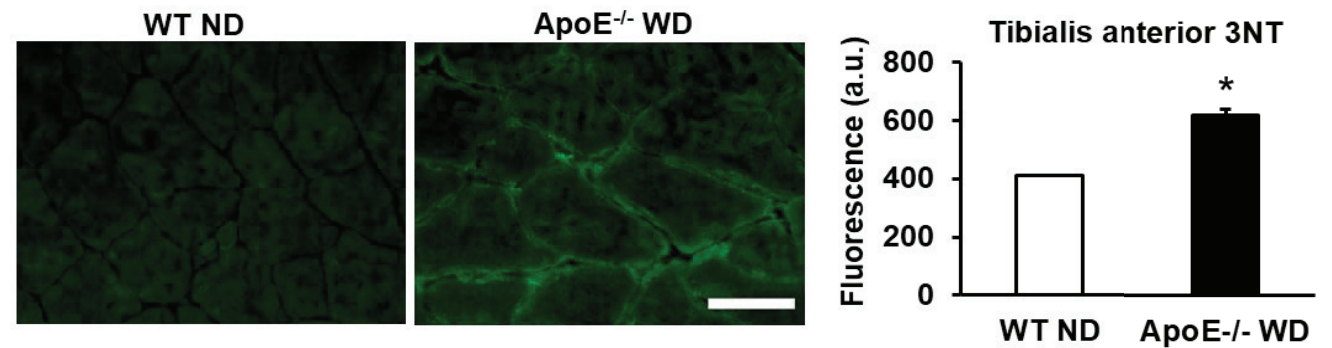

E

Tibialis anterior $4 \mathrm{HNE}$
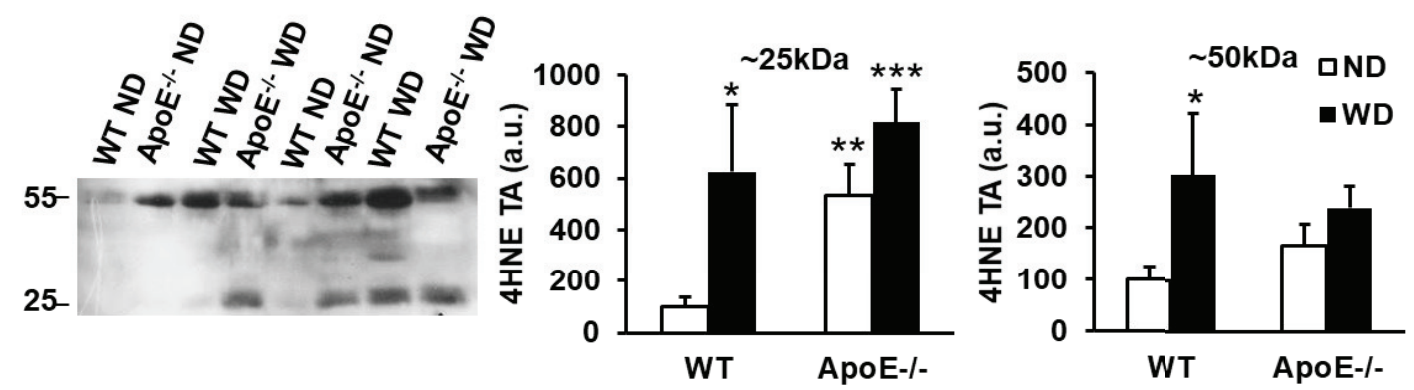


\section{Supplementary Fig. 5. ApoE deficiency and Western-type diet independently}

increase oxidative stress in tibialis anterior. Oxidative stress in skeletal muscle was assessed in $\mathrm{C} 57 \mathrm{BI} / 6 \mathrm{~J}$ (wild-type, WT) and $\mathrm{ApoE}^{-/}$mice fed a standard chow diet (normal diet, ND) or Western-type diet (WD) for 12 weeks. (A) Superoxide production as measured by LC-MS. Data are expressed as mean \pm SD in arbitrary units and shown as relative fold increase to WT ND after normalisation to protein content. (B) Representative images for free radical staining with $10 \mu \mathrm{M}$ DHE and treated with $400 \mathrm{U} / \mathrm{mL}$ PEG-SOD prior to DHE staining (x20 magnification, scale bar $100 \mu \mathrm{m}$ ). (C) Protein carbonylation in lysates from tibialis anterior as determined by immunoblot. Data are expressed as mean \pm SD and data are shown as relative fold increase to WT ND after normalisation to total protein. (D) Immunoblot in protein lysates of tibialis anterior for tyrosine nitration (3NT) and immunostaining for 3NT (x40 magnification, scale bar $50 \mu \mathrm{m}$ ). Data are expressed as mean \pm SD. For immunoblotting data are shown as relative fold increase to WT ND after normalisation to $\beta$ tubulin. (E) Western blot in protein lysates of tibialis anterior for $4 \mathrm{HNE}$ adducts in histidine residues. Data are expressed as mean $\pm \mathrm{SD}$ and shown as relative fold increase to WT ND after normalisation to $\beta$ tubulin. Statistical analysis was performed by two-way ANOVA (Main effects: Genotype: $p<0.05$ for protein carbonylation, $3 \mathrm{NT}$ at $50 \mathrm{kDa}$ and4HNE at $25 \mathrm{kDa}$; Diet $\mathrm{p}<0.01$ for $3 \mathrm{NT}$ and $4 \mathrm{HNE}$; Interaction; $p<0.05$ for protein carbonylation) followed by Bonferroni post hoc tests, ${ }^{*} p<0.05,{ }^{* *} p<0.01,{ }^{* * *} p<0.001$ vs WT ND ( $n=6 /$ group). Statistical analysis for $3 N T$ staining was performed by Mann-Whitney $U$ test, ${ }^{*} p<0.05$. 
A
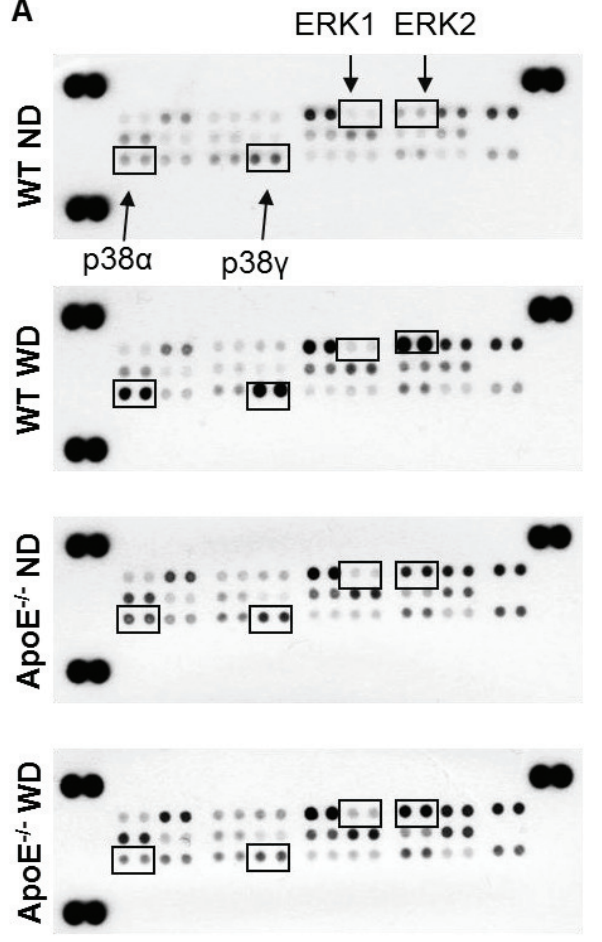

\begin{tabular}{|c|c|c|c|}
\hline \multirow[t]{2}{*}{ MAPKs } & \multicolumn{3}{|c|}{ Fold Changes } \\
\hline & WT WD & ApoE $E^{-/-}$ND & ApoE $^{-/-}$WD \\
\hline Akt1 & 1.65 & 1.57 & 2.13 \\
\hline Akt2 & 1.27 & 1.51 & 1.91 \\
\hline Akt3 & 1.39 & 1.24 & 2.07 \\
\hline Akt Pan & 1.55 & 1.31 & 2.46 \\
\hline CREB & 1.57 & 1.29 & 1.60 \\
\hline ERK1 & 1.69 & 1.42 & 2.50 \\
\hline ERK2 & 31.29 & 19.19 & 27.69 \\
\hline GSK3 $\alpha / \beta$ & 1.48 & 1.35 & 1.68 \\
\hline GSK3 $\beta$ & 1.14 & 1.13 & 1.23 \\
\hline JNK1 & 0.90 & 1.04 & 1.74 \\
\hline JNK2 & 0.90 & 1.01 & 1.48 \\
\hline JNK pan & 1.4 & 1.1 & 1.7 \\
\hline MKK3 & 1.19 & 1.16 & 1.68 \\
\hline MKK6 & 2.59 & 1.31 & 2.30 \\
\hline MSK2 & 1.44 & 1.23 & 2.09 \\
\hline p38a & 2.75 & 1.21 & 0.98 \\
\hline p38ס & 0.74 & 0.95 & 1.04 \\
\hline p38y & 2.64 & 0.95 & 0.84 \\
\hline p53 & 0.66 & 0.98 & 1.02 \\
\hline p70S6K & 1.06 & 0.93 & 1.04 \\
\hline RSK2 & 1.53 & 0.77 & 1.27 \\
\hline TOR & 1.01 & 1.39 & 1.49 \\
\hline
\end{tabular}
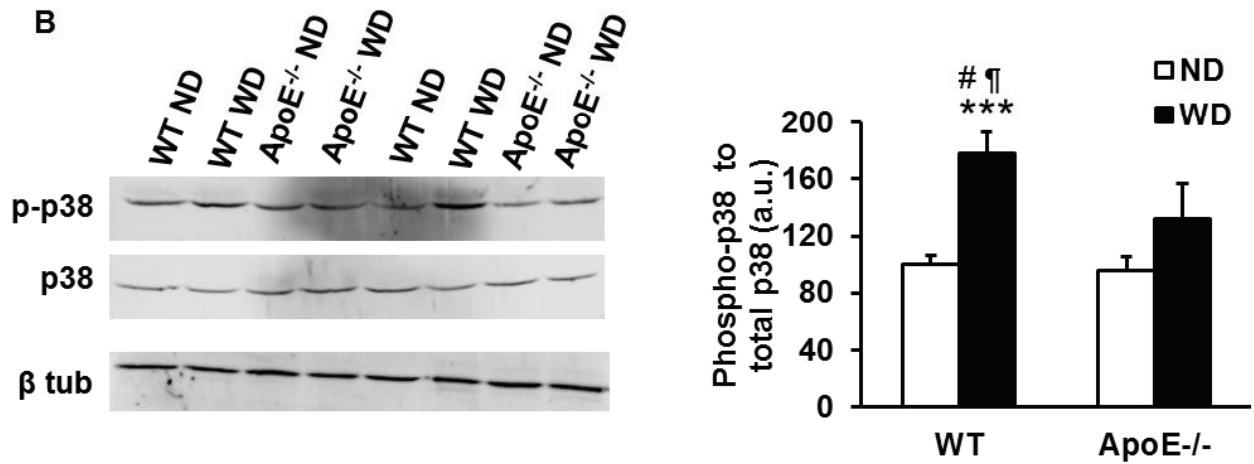

Supplementary Fig. 6. Western-type diet and ApoE deficiency modulate independently the activation of MAPKs in skeletal muscle. Phosphorylation levels of various MAPKs were assessed in skeletal muscle of C57BI/6J (wild-type, WT) and ApoE ${ }^{-/-}$ mice fed a standard chow diet (normal diet, ND) or Western-type diet for 12 weeks.(A) Results of Phospho-MAPK antibody array (R\&D). The densitometric analysis was presented as fold change. Fold change was calculated as: the ratio of each of the 3 groups to WT ND group (n=3/group). (B) Immunoblot for phospho-p38 and p38. Data are expressed as mean \pm SD and shown as the ratio of phosphor-p38 to p38 after normalisation to $\beta$ tubulin. Statistical analysis was performed by two-way ANOVA (Main effects: Genotype: $p>0.05$ genotype; Diet $p<0.05$ phospho-38; Interaction; $p>0.05$ for 
phosho-p38) followed by Bonferroni post hoc tests, ${ }^{* * *} p<0.001$ vs WT ND, $\# p<0.05$ vs ApoE $^{-/-}$ND and $\uparrow p<0.05$ vs ApoE ${ }^{-/-}$WD (n=4/group). 

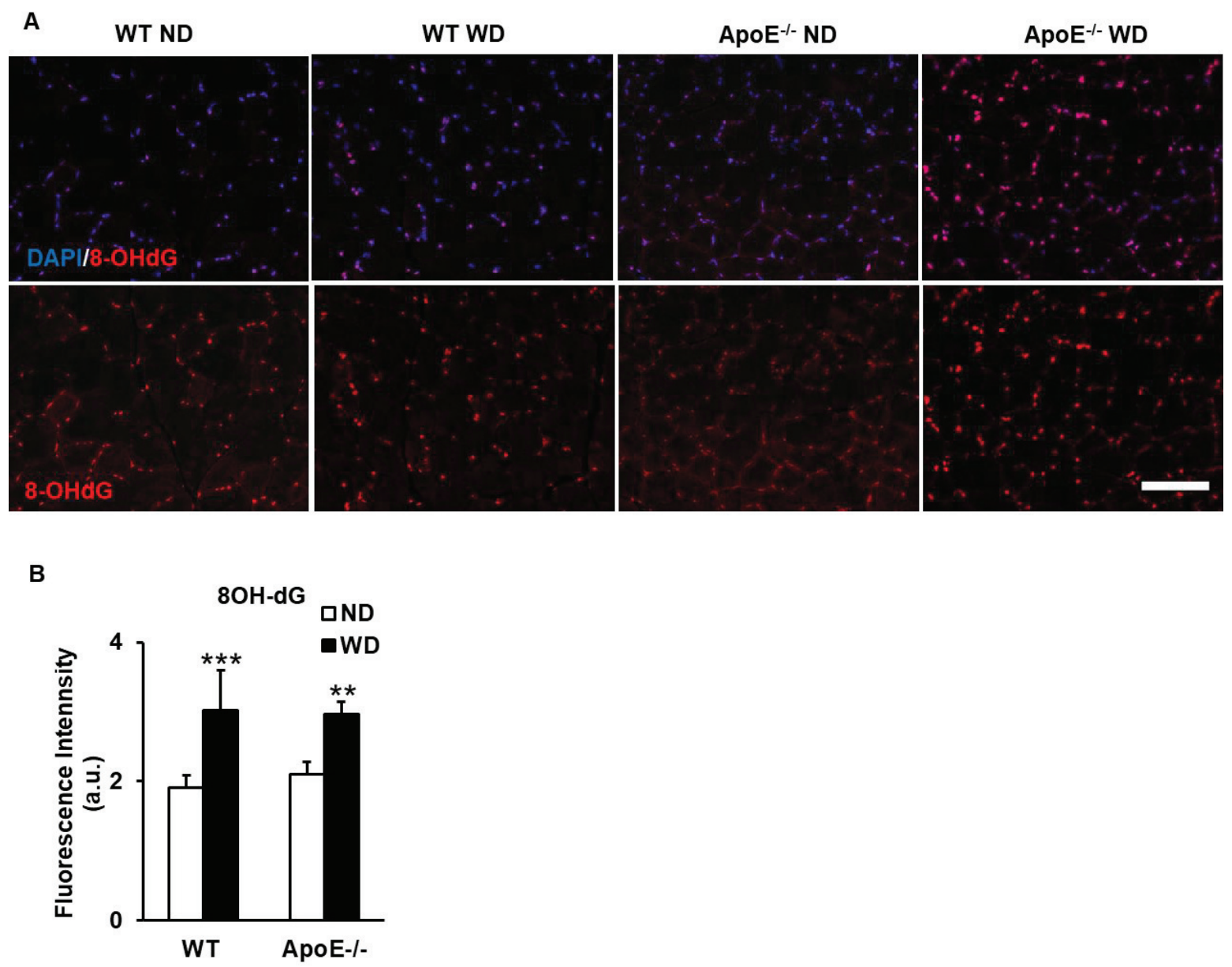

Supplementary Fig. 7. Western-type diet but not ApoE deficiency induces guanosine oxidative damage induced from western-type diet in skeletal muscle. DNA damage was assessed in skeletal muscle of C57BI/6J (wild-type, WT) and ApoE ${ }^{-/}$ mice fed a standard chow diet (normal diet, ND) or Western-type diet for 12 weeks. (A) Representative images of 8-OHdG and DAPI staining and 8-OHdG staining only in tibialis anterior (x20 magnification, scale bar $100 \mu \mathrm{m}$ ). (B) Quantification of fluorescence intensity of $8-\mathrm{OHdG}$ in the tibialis anterior. Data are expressed as mean \pm SD. Statistical analysis was performed by two-way ANOVA (Main effects: Diet $p<0.001$ ) followed by Bonferroni post hoc tests, ${ }^{* *} p<0.01$ and ${ }^{* * *} p<0.001$ vs WT ND ( $n=6 /$ group). 

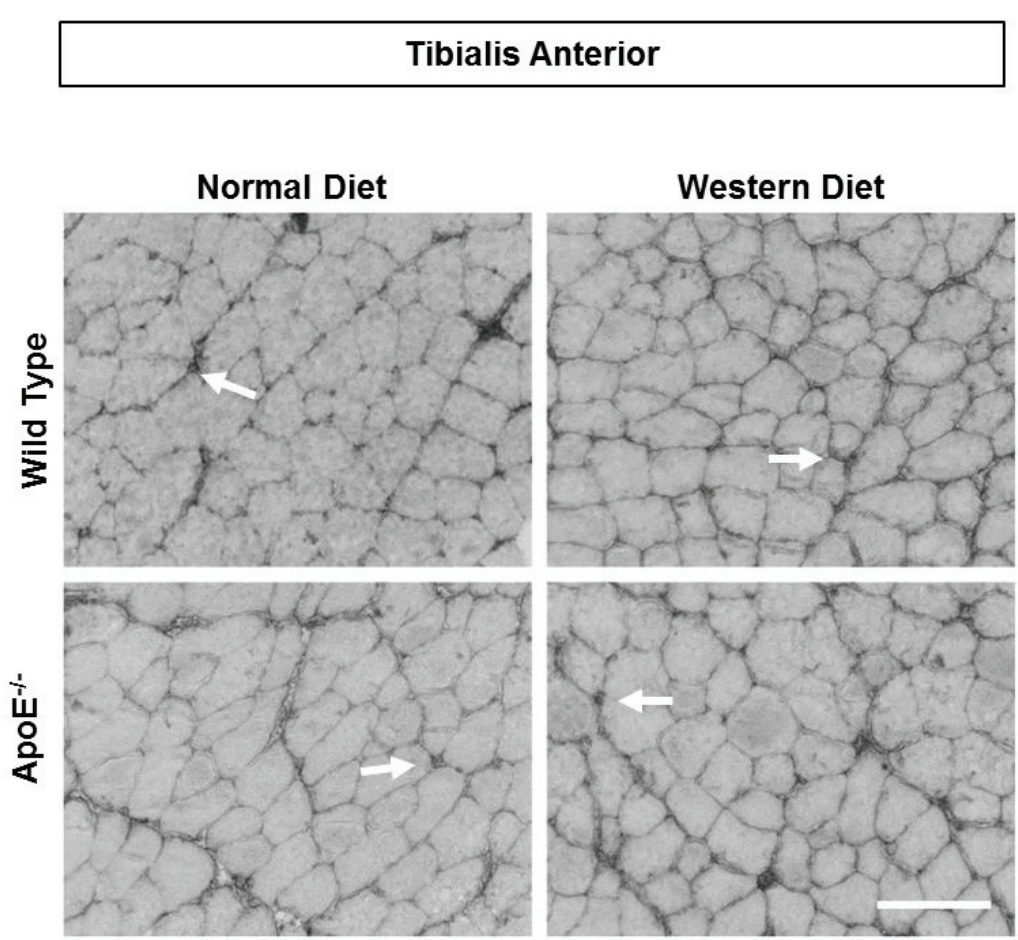

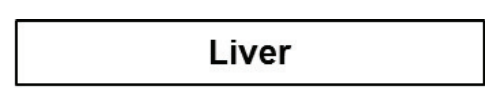

Wild type Normal Diet

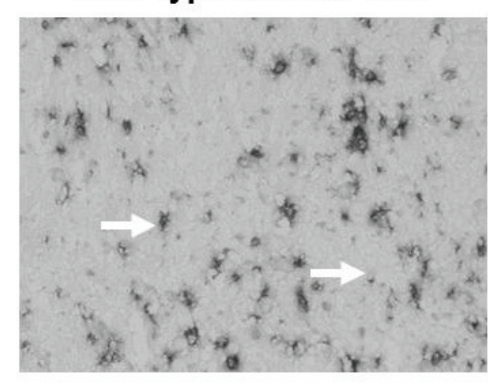

Supplementary Fig. 8. ApoE deficiency and Western-type diet do not induce macrophage infiltration. Representative images for F4.80 staining in tibialis anterior (x20 magnification, scale bar $100 \mu \mathrm{m}$ ). Arrows depict macrophages in skeletal muscle. Macrophages were in low abundance on skeletal muscle as compared to liver tissue. 
A Normal Diet

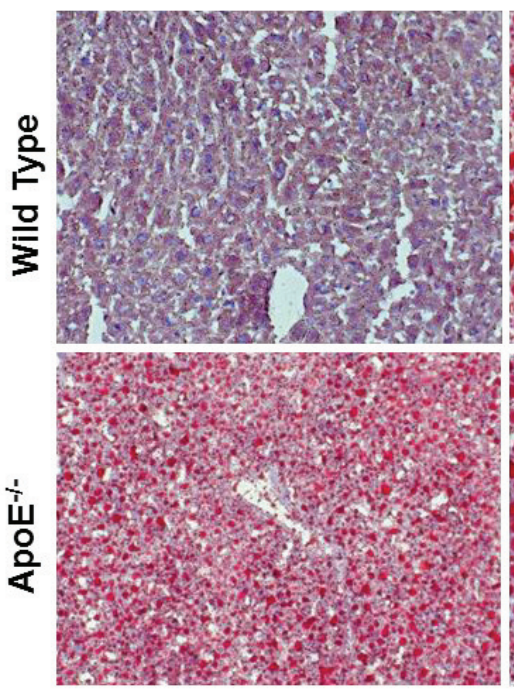

B

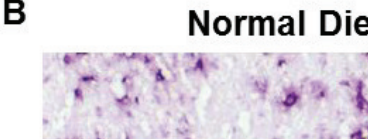

Western Diet
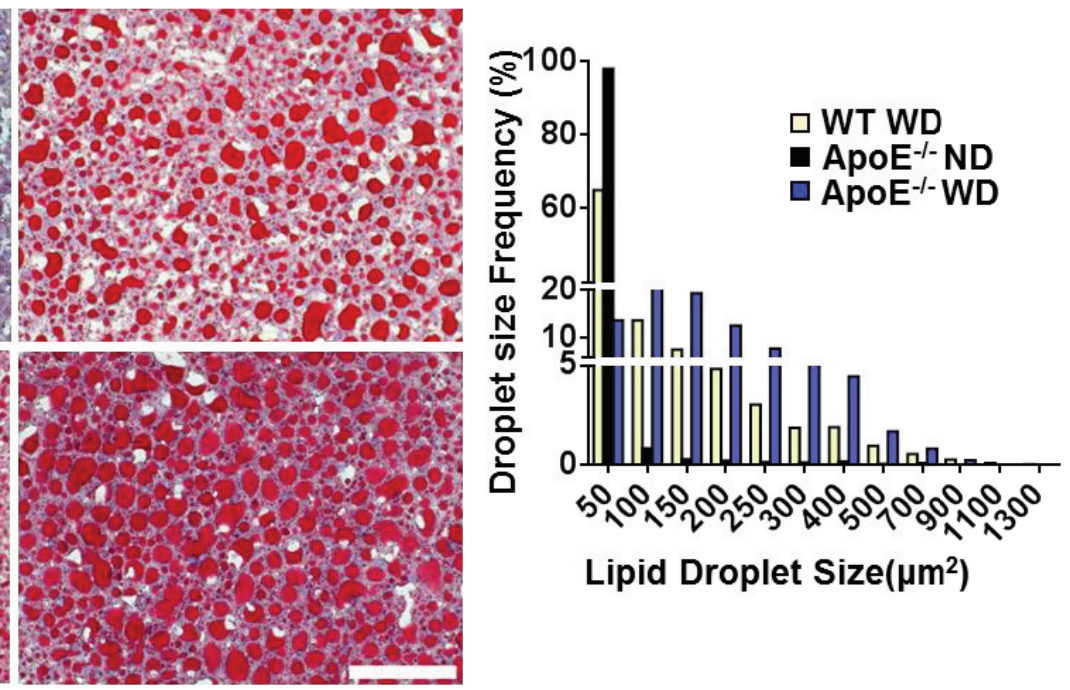

Lipid Droplet Size $\left(\mu \mathrm{m}^{2}\right)$

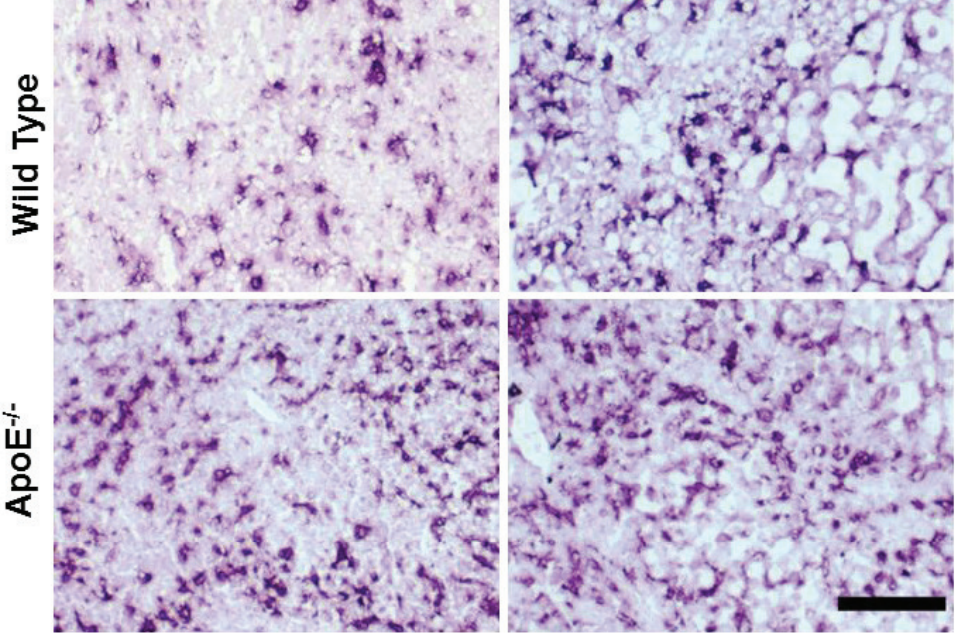

Western Diet

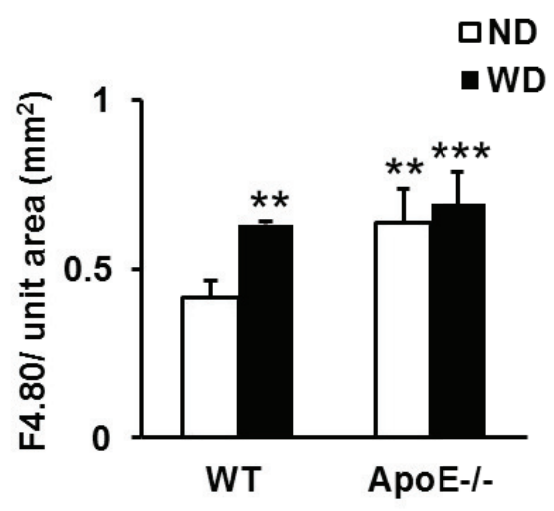

C

口WT ND

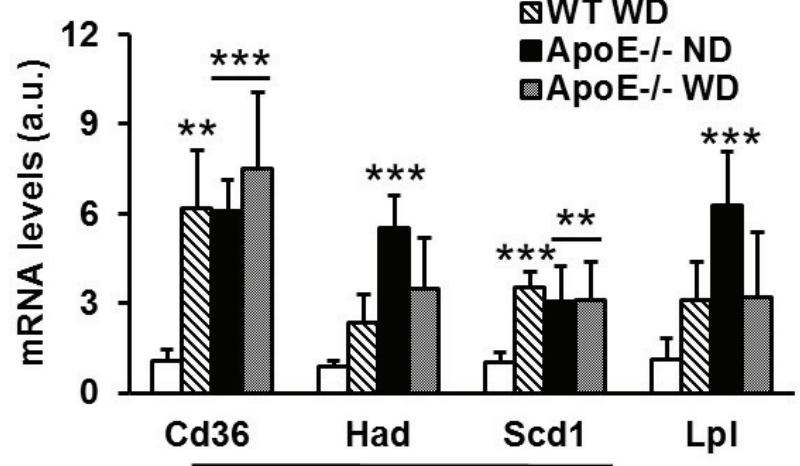

Fatty acid uptake and oxidation

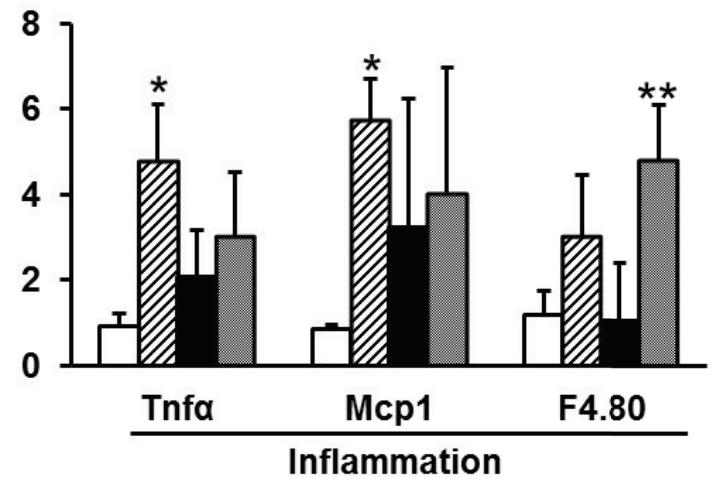


Supplementary Fig. 9. ApoE deficiency and Western-type diet independently induce hepatic fat accumulation and inflammation. Hepatic ectopic fat accumulation and inflammation was assessed in C57BI/6J (wild-type, WT) and $\mathrm{ApoE}^{-/-}$mice fed a standard chow diet (normal diet, ND) or Western-type diet for 12 weeks. (A) Representative images of Oil Red $O$ staining in the liver and quantification of lipid droplet size and frequency $(x 20$ magnification, scale bar $100 \mu \mathrm{m}$ ). Data are expressed as percentages of lipid droplet size distribution. (B) Representative images of F4.80 staining in the liver and relative quantification of macrophages per area (x20 magnification, scale bar $100 \mu \mathrm{m}$ ). Data are expressed as mean \pm SD. (C) mRNA levels of genes involved in fatty acid uptake and oxidation, and inflammatory genes. Data are expressed as mean \pm SD and shown as fold increase relative to WT ND in arbitrary units. Statistical analysis was performed by $X^{2}$ square distribution for lipid droplet size distribution and two-way ANOVA (Main effects: Genotype: $\mathrm{p}<0.01$ for Cd36, Had, Lpl, and F4.80 and F4.80 staining; Diet $\mathrm{p}<0.05$ for F4.80 staining and all genes except for $L p /$ and $F 4.80$; Interaction; $p<0.05$ for F4.80 staining and for all genes, except for F4.80) followed by Bonferroni post hoc tests for gene expression and inflammation, ${ }^{*} p<0.05,{ }^{* *} p<0.01,{ }^{* * *} p<0.001$ vs WT ND (n=6/group). For lipid droplet size distribution: $\quad x^{2}=150.877 ; \mathrm{df}=6 ; \mathrm{p}<0.001$ for $\mathrm{ApoE}^{-/-}$WD versus $\mathrm{ApoE}^{-/} \mathrm{ND}$, $X^{2}=42.424 ; \mathrm{df}=6 ; p<0.001$ for $W T$ WD versus $A p o E^{-/} \mathrm{ND}$ and $X^{2}=54.903 ; \mathrm{df}=5 ; \mathrm{p}<0.001 \mathrm{ApoE}^{-/-}$ WD versus WT WD (n=6/group). 
A

Normal Diet

Western Diet
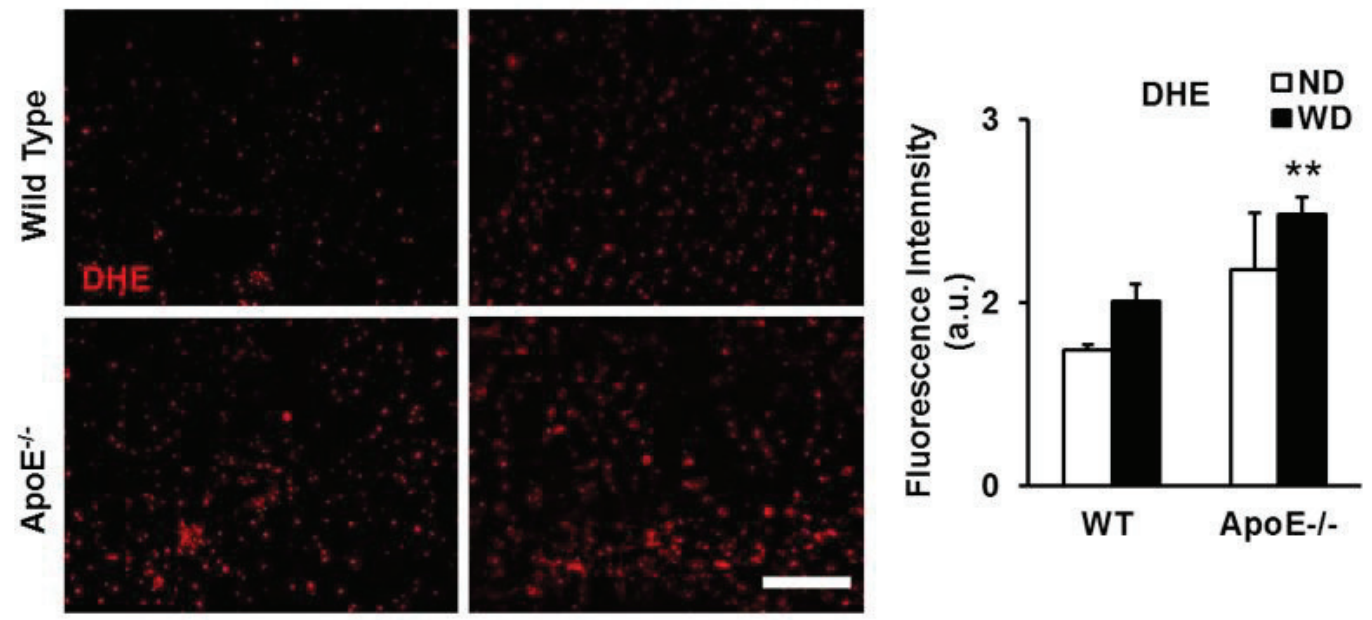

B
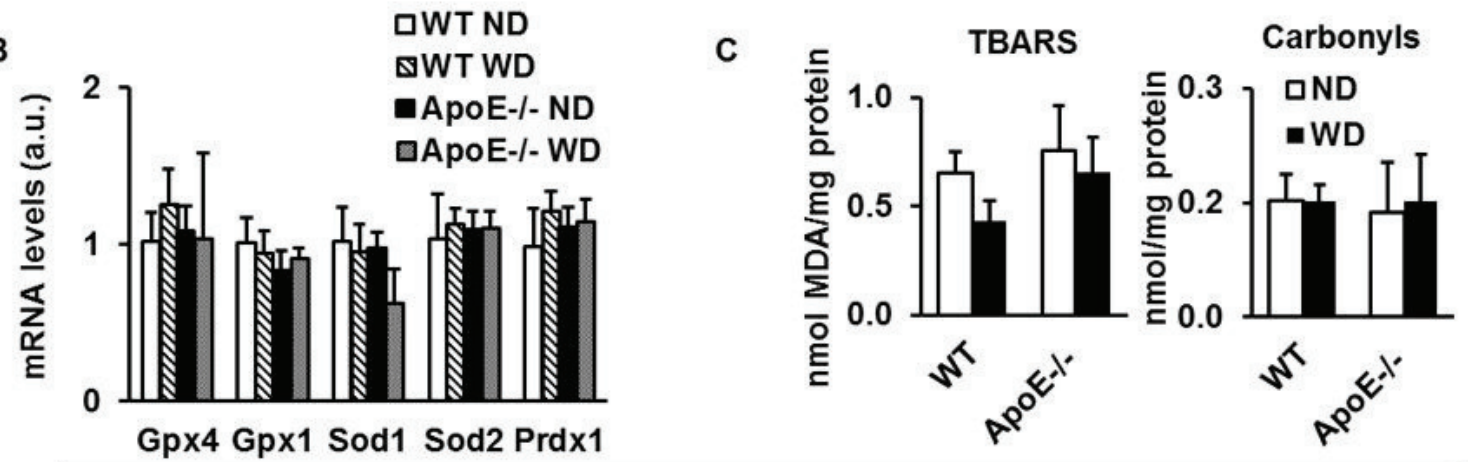

D
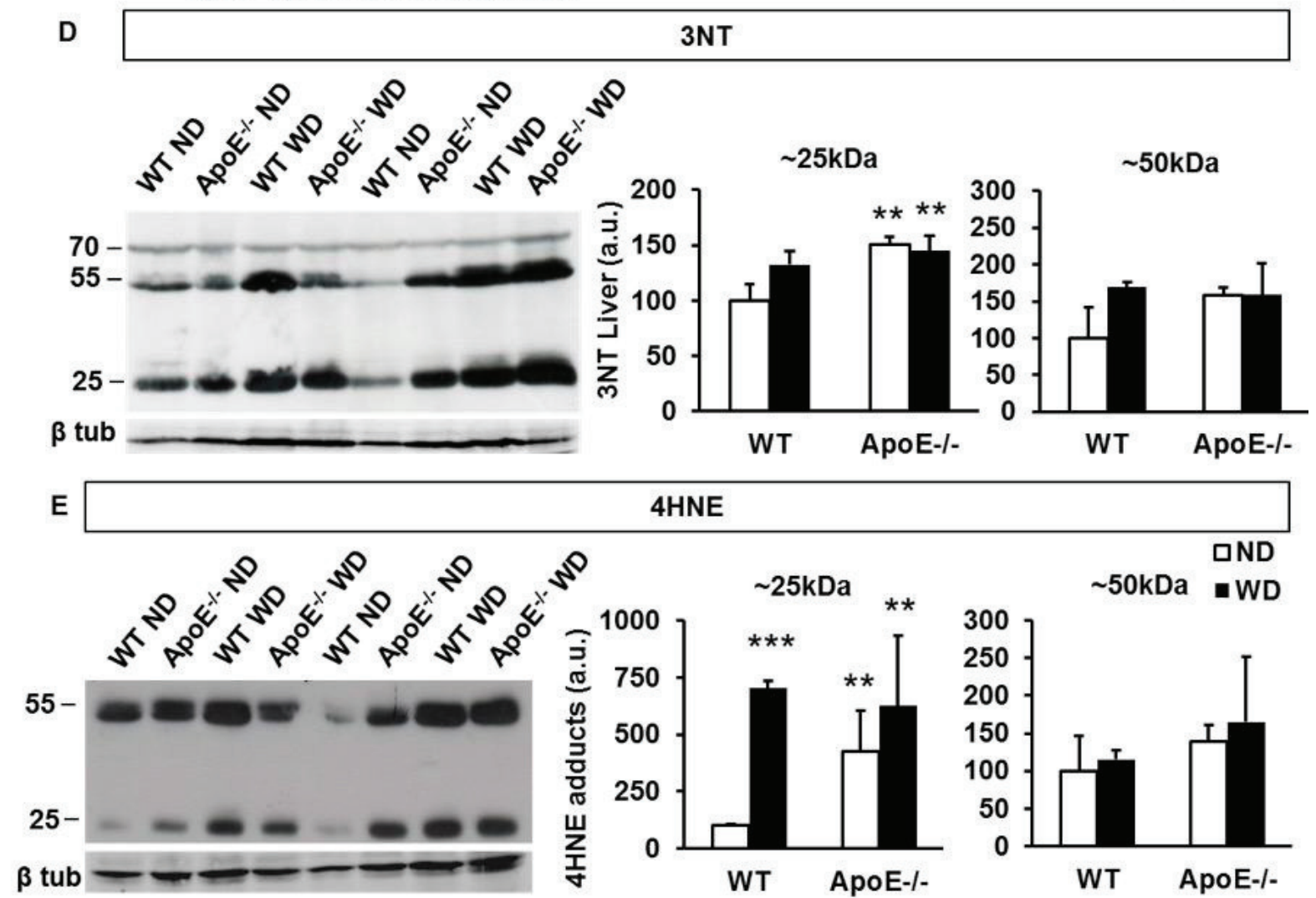
Supplementary Fig. 10. ApoE deficiency and Western-type diet increase hepatic oxidative protein modifications. Hepatic oxidative stress was assessed in C57BI/6J (wildtype, WT) and $\mathrm{ApoE}^{-/-}$mice fed a standard chow diet (normal diet, ND) or Western-type diet (WD) for 12 weeks. (A) Representative images of free radical staining with $10 \mu \mathrm{M}$ DHE in the liver (x20 magnification, scale bar $100 \mu \mathrm{m}$ ) and quantification of fluorescence intensity. (B) mRNA levels of antioxidant genes. Data are expressed as mean \pm SD and shown as fold increase relative to WT ND in arbitrary units. (C) TBARS assay and protein carbonyl content in liver. Data are expressed as mean \pm SD. (D) Immunoblot in protein lysates of liver for tyrosine nitration (3NT). Data are expressed as mean \pm SD and shown as fold increase relative to WT ND group after normalisation to $\beta$ tubulin. (E) Immunoblot for 4 HNE adducts in histidine residues. Data are expressed as mean \pm SD and shown as fold increase relative to WT ND group after normalisation to $\beta$ tubulin. Statistical analysis was performed by twoway ANOVA (Main effects: Genotype: $p<0.01$ for DHE fluorescence and 3NT; Diet $p<0.05$ for DHE fluorescence and $4 \mathrm{HNE}$; Interaction; $p<0.05$ for $3 \mathrm{NT}$ and $4 \mathrm{HNE}$ ) followed by Bonferroni post hoc tests, ${ }^{* *} p<0.01$ and ${ }^{* * *} p<0.001$ vs WT ND ( $n=6 /$ group). 


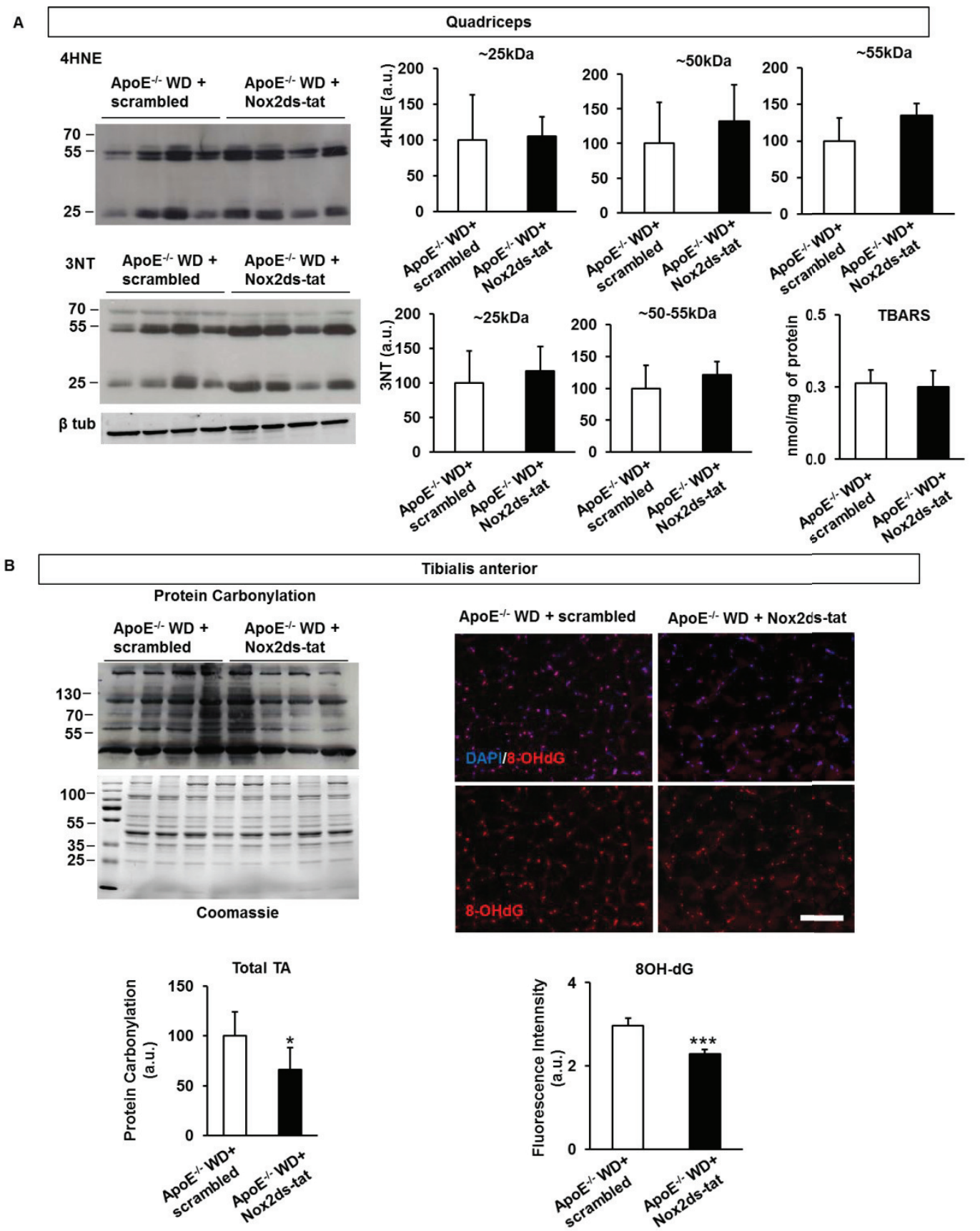

Supplementary Fig. 11. Nox2ds-tat peptide decreases oxidative stress in skeletal muscle. Oxidative stress was assessed in skeletal muscle of $\mathrm{ApoE}^{-{ }^{--}}$mice fed Westerntype diet for 12 weeks and treated with either Nox2ds-tat peptide (ApoE ${ }^{--}$WD + Nox2dstat) or scrambled peptide for the last 4 weeks of feeding (ApoE ${ }^{-/}$WD + scrambled). (A) Immunoblot in protein lysates of quadriceps for 3NT and 4HNE adducts in histidine residues. Data are expressed as mean \pm SD and data are shown as relative fold change to $A p o E^{-/-}$WD treated with scrambled sequence after normalisation to $\beta$ tubulin. (B) Immunoblot in protein lysates of tibialis anterior for protein carbonylation. Data are expressed as mean $\pm S D$ and data are shown as relative fold change to $A p o E^{-/-} W D$ treated with scrambled sequence after normalisation to total protein content. 
Representative images of 8-OHdG and DAPI staining and 8-OHdG staining only in tibialis anterior (x20 magnification, scale bar $100 \mu \mathrm{m}$ ) and quantification of 8-OHdG fluorescence intensity. Data are expressed as mean \pm SD. Statistical analysis was performed by Student's t test, ${ }^{*} p<0.05$ vs $A_{p o E^{-/-}} W D$ treated with scrambled sequence ( $n=6 /$ group). 
A
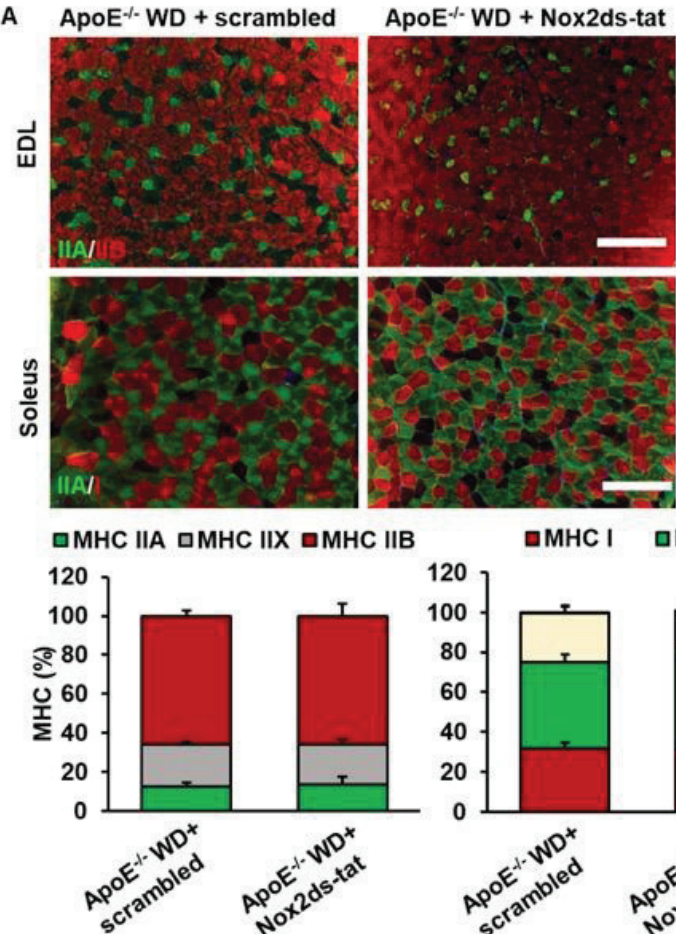

C

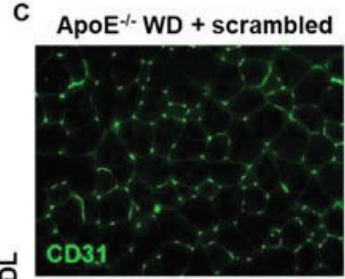

㟶
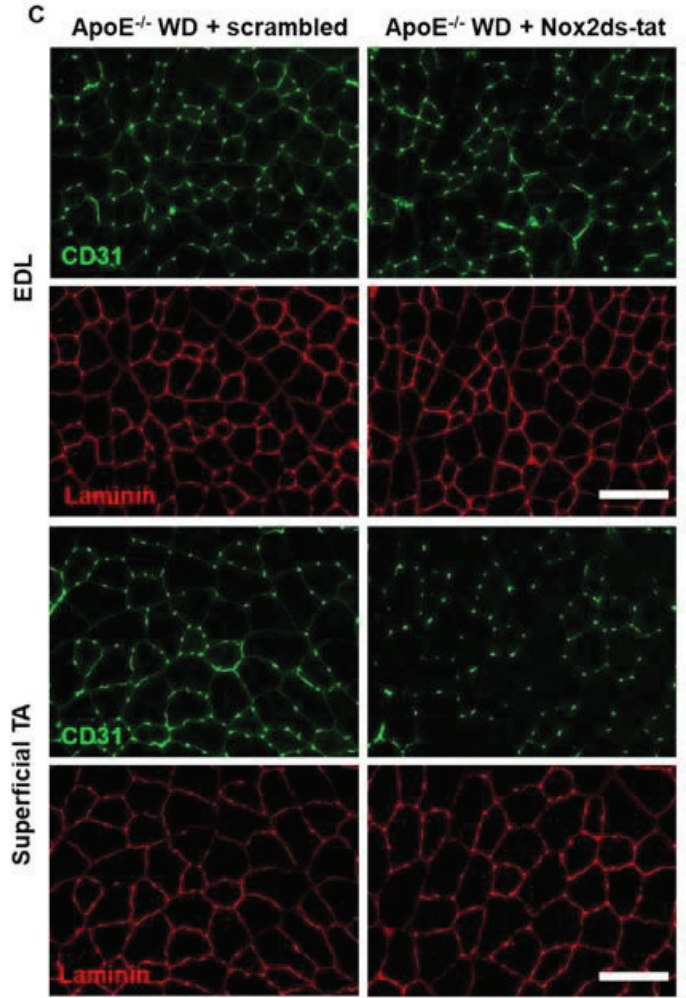

B

峞

ApoE $^{-/}$WD + scrambled $\quad$ ApoE ${ }^{-/}$WD + Nox2ds-tat

응

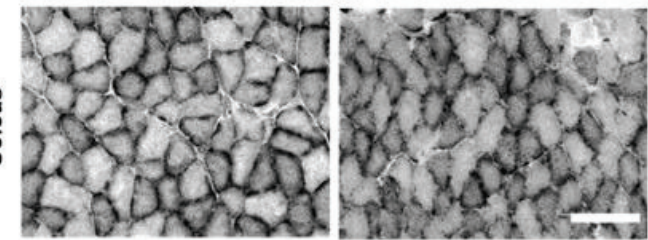

EDL

Soleus

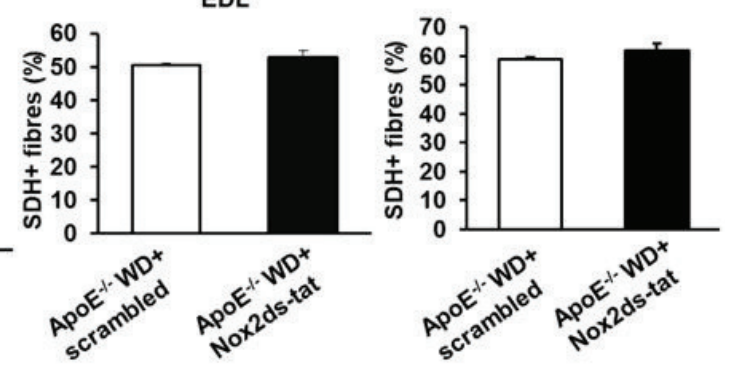

D
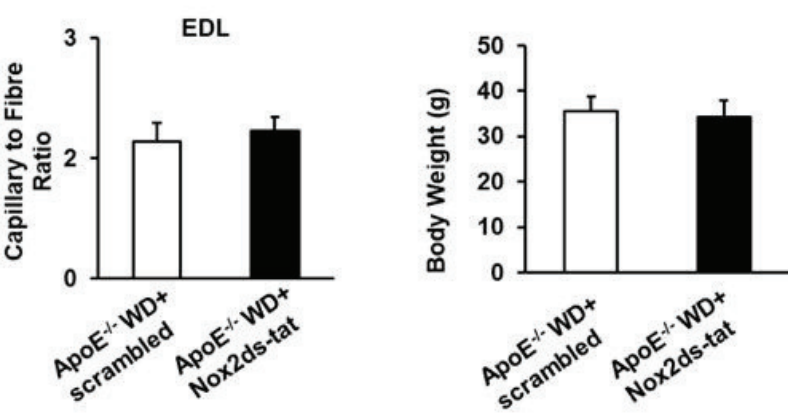

E

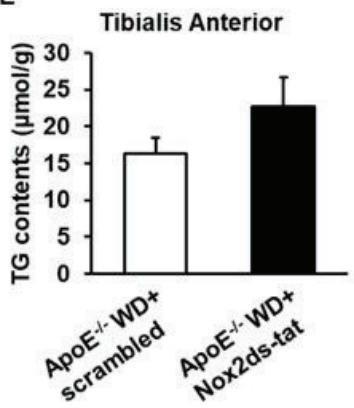

Supplementary Fig. 12. Nox2ds-tat peptide does not affect intramyocellular ectopic fat accumulation, capillary density or myofibre composition. Ectopic fat accumulation, myofibre composition, mitochondrial activity and capillary density was assessed in skeletal muscle of $\mathrm{ApoE}^{-/}$mice fed Western-type diet for 12 weeks and treated either with Nox2ds-tat peptide $\left(\mathrm{ApoE}^{-/-} \mathrm{WD}+\right.$ Nox2ds-tat) or scrambled peptide (ApoE ${ }^{-/}$WD + scrambled) for the last 4 weeks of feeding. (A) Representative images of 
MHC immunostaining and quantification of myofibre type in EDL and soleus (x10 magnification, scale bar $200 \mu \mathrm{m}$ ). Data are expressed as mean \pm SD. (B) Representative images of SDH staining and quantification of positive myofibres in EDL and soleus (x20 magnification, scale bar $100 \mu \mathrm{m}$ ). Data are expressed as mean \pm SD. (C) Representative images of CD31 immunostaining and quantification of relative capillary density by Axiovision software in EDL and superficial tibialis anterior (x20 magnification, scale bar $100 \mu \mathrm{m})$. Data are expressed as mean \pm SD and shown as relative fold increase to ApoEI- WD treated with scrambled sequence. (D) Body weight of $A p o E^{-/-}$WD treated with scrambled sequence or Nox2ds-tat. (E) Triacylglycerol content in tibialis anterior as determined by Gas chromatography. Data are expressed as mean \pm SD. Statistical analysis was performed by Student's t test ( $n=6 /$ group). 